\title{
Monte Carlo and Tissue-Phantom studies of Photoacoustic Imaging of Carotid Intraplaque Haemorrhage
}

\author{
by \\ Ahmed Yahia Khiari \\ Master of Science, University of New Brunswick, 2015 \\ Bachelor of Science, King Fahd University of Petroleum and Minerals, 2011
}

\author{
A thesis \\ presented to Ryerson University \\ in partial fulfillment of the \\ requirements for the degree of \\ Master of Science
}

In the Program of

Biomedical Physics

Toronto, Ontario, Canada, 2019

(c)Ahmed Yahia Khiari, 2019 


\section{Author's Declaration}

I hereby declare that I am the sole author of this thesis. This is a true copy of the thesis, including any required final revisions, as accepted by my examiners.

I authorize Ryerson University to lend this thesis to other institutions or individuals for the purpose of scholarly research.

I further authorize Ryerson University to reproduce this thesis by photocopying or by other means, in total or in part, at the request of other institutions or individuals for the purpose of scholarly research.

I understand that my thesis may be made electronically available to the public.

AHMED YAHIA KHIARI 


\section{Abstract}

Strokes are the second leading cause of death worldwide. Vulnerable carotid plaques are a primary cause of stroke, with carotid Intraplaque Haemmorrghages being a key feature of vulnerability. MR imaging, which is sensitive to the presence of endogenous Methemoglobin (MetHb), is used to detect IPH. MRI is however expensive, and not readily available. We propose the use of Photoacoustic Imaging (PAI) for the detection of IPH, with MetHb being the primary PA imaging target. I examine the feasibility of this approach by performing Monte Carlo studies of light propagation, energy deposition and PA generation in tissuemimicking models, as well as experimental PA measurements of MetHb in tissue-mimicking phantoms. I show that is possible to achieve an SNR of $\sim 50 \mathrm{~dB}$ at the average carotid artery depth of $\sim 21 \mathrm{~mm}$, with the possibility of imaging up to $\geq 32 \mathrm{~mm}$ in Type I skin using commonly available hardware. 


\section{Table of Contents}

Author's Declaration $\quad$ ii

$\begin{array}{ll}\text { Abstract } & \text { iii }\end{array}$

Table of Contents vii

$\begin{array}{ll}\text { List of Tables } & \text { ix }\end{array}$

$\begin{array}{ll}\text { List of Figures } & \text { xvi }\end{array}$

$\begin{array}{ll}\text { Abbreviations } & \text { xvii }\end{array}$

1 Introduction $\quad 1$

1.1 Overview of Strokes . . . . . . . . . . . . . . . . . . . . 1

1.1.1 Health and Economic Impact of Strokes . . . . . . . . . . 1

1.1.2 Strokes: Causes, Risk Factors, and Treatment . . . . . . . . . . 2

1.1.3 Carotid Plaque Vulnerability . . . . . . . . . . . . . . . . . 4

1.1.3.1 The Process of Arteriosclerosis . . . . . . . . . . 4

1.1.3.2 Stable vs. Unstable Plaques . . . . . . . . . . . . . 4

1.1.4 IPH and Recurrent Strokes . . . . . . . . . . . . . . 5

1.1.5 Non-invasive Imaging of Vulnerable Plaques and IPH . . . . . . 6

1.1.6 An Alternative to MRI . . . . . . . . . . . . . . 7

1.2 Principles of Photoacoustic Imaging . . . . . . . . . . . . . . . . . 9 
$1.2 .1 \quad$ Photoacoustic Effect . . . . . . . . . . . . . . . . . . 9

1.2 .2 Why Photoacoustic Imaging? . . . . . . . . . . . . . . . . 12

1.2 .3 Light interactions in tissue . . . . . . . . . . . . . . . . 12

1.2.4 Biomedical Applications of Photoacoustics . . . . . . . . . . . 13

1.3 Motivation, Hypothesis and Specific Aims _. . . . . . . . . . . 15

1.4 Thesis Outline . . . . . . . . . . . . . . . . . . . . . . 17

2 Methods and Tools 18

2.1 Determining Tissue Properties . . . . . . . . . . . . . . . . . . 18

$2.1 .1 \quad$ Neck Tissues . . . . . . . . . . . . . . . . . . . . . . 18

$2.1 .2 \quad$ Plaque and IPH . . . . . . . . . . . . . . . . . . 20

2.2 Monte Carlo Simulation . . . . . . . . . . . . . . . . . . . . . 23

2.2 .1 Simulation Parameters . . . . . . . . . . . . . . . . . 23

2.2 .2 Data acquisition and analysis . . . . . . . . . . . 23

2.3 Experimental Photoacoustic Measurements . . . . . . . . . . . . . . . . . 24

2.3 .1 Tissue-mimicking phantoms . . . . . . . . . . . . . . . . 24

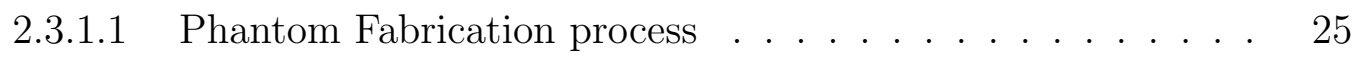

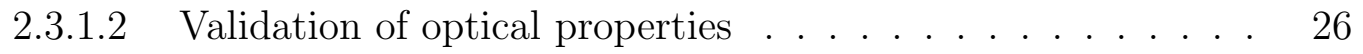

2.3.1.3 Methaemoglobin as absorbing target . . . . . . . . 28

$2.3 .2 \quad$ Experimental PA setup . . . . . . . . . . . . . . . . . . 30

2.3 .3 Measurement and Analysis . . . . . . . . . . . . . . . . . . 31

2.3.3.1 Data Acquisition . . . . . . . . . . . . . . . 31

2.3.3.2 Data Analysis . . . . . . . . . . . . . . . 32

3 Results 35

3.1 Properties of Phantoms and MetHb . . . . . . . . . . . . . . . 35

3.1.1 Absorption spectra of Whole Blood and MetHb . . . . . . . . 35

3.1 .2 Measured spectra of tissue-mimicking phantoms . . . . . . . . . 36 
3.1 .3 US Images of tissue-mimicking phantoms . . . . . . . . . . . . 36

3.2 Monte Carlo Simulation Results . . . . . . . . . . . . . . . . . . . . . . 39

3.3 Experimental Measurements . . . . . . . . . . . . . . . . . . . . 45

$\begin{array}{lll}4 & \text { Discussion } & 49\end{array}$

4.1 Assumptions and Limitations . . . . . . . . . . . . . . . . . . . 49

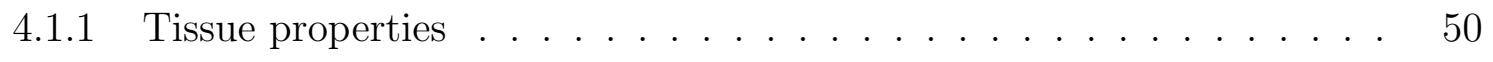

$4.1 .2 \quad$ Plaque, IPH and MetHb . . . . . . . . . . . . . 50

4.1 .3 Acoustic Attenuation, Noise and Detection . . . . . . . . . . 51

4.1.3.1 Acoustic attenuation . . . . . . . . . . . . . 51

4.1.3.2 Detector location . . . . . . . . . . . . . 51

4.1.3.3 Anatomical noise and artifacts . . . . . . . . . 52

4.1.3.4 Detection limits . . . . . . . . . . . . . . 52

$4.1 .4 \quad$ Laser setup . . . . . . . . . . . . . . . . . . . . 53

4.2 Corrections for Maximum Imaging Depth . . . . . . . . . . . . . 53

4.2 .1 Optical attenuation corrections $\ldots \ldots \ldots \ldots \ldots \ldots$

$4.2 .2 \quad$ Acoustic attenuation corrections . . . . . . . . . . . . . 55

4.3 Simulation vs. Experiment . . . . . . . . . . . . . . . 56

5 Conclusions and Future Work $\quad 59$

5.1 Thesis Conclusions . . . . . . . . . . . . . . . . . . . . 59

5.2 Future Directions . . . . . . . . . . . . . . . . . . . 61

$\begin{array}{ll}\text { A MR Images at the carotid bifurcation } & 63\end{array}$

$\begin{array}{ll}\text { B The Inverse Adding-Doubling Method } & 66\end{array}$

$\begin{array}{lc}\text { C Monte Carlo Simulation Results } & 69\end{array}$

$\begin{array}{ll}\text { D Sample measurements of TT and DR } & 75\end{array}$ 
Bibliography 


\section{List of Tables}

2.1 Dimensions and optical properties at $630 \mathrm{~nm}$ of the proposed tissue-mimicking

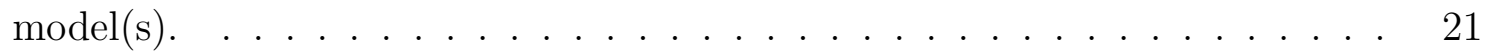

2.2 Volume fractions defined as volume of ingredient $V_{i}$ divided by the total volume of solution $V_{T}: \frac{V_{i}}{V_{T}} \%$ of the ingredients used to create the skin/fat and muscle layers in the tissue-mimicking phantoms. . . . . . . . . . . . . . 27

2.3 A list of the phantom/tissue models created for ${ }^{1}$ Monte Carlo and ${ }^{2}$ experimental studies. . . . . . . . . . . . . . . . . . .

3.1 A summary of the measured average optical properties compared to the target optical properties of fat and muscle tissue layers. The absolute $\%$ difference off the reference values range between $0.83 \%$ for muscle absorption and $18.9 \%$ for muscle reduced scattering. . . . . . . . . . . . . . . . . . . . 38

3.2 Monte Carlo simulated PA amplitudes $(\mathrm{dB})$ in 8 tissue models normalized to the maximum in the $5 \mathrm{~m} 5 \mathrm{f}$ simulation. . . . . . . . . . . . . .

3.3 Averaged experimentally measured peak SNR(dB) for different phantoms and MetHb absorptions. The values in parentheses define the maximum number of peak SNRs per measurement averaged to achieve a coefficient of variation $C V \leq 10 \%$. The blank table cells indicate an absence of valid measurement.

3.4 The attenuation of SNR with fat thickness given by the experimental slopes in $\mathbf{d B} \cdot \mathbf{m m}^{-\mathbf{1}}$ for different MetHb absorptions. . . . . . . . . . . . . . . 
4.1 Different skin types with the corresponding $60 \mu m$ epidermis absorption and predicted maximum imaging depth. Per the Fitzpatrick scale, lightly-pigmented skins are referred to as types I and II, moderately-pigmented as types III and IV and darkly-pigmented as types V and VI. The epidermal absorption values were reproduced from $[53] \ldots \ldots \ldots$. . . . . . . . . . . . . . 55

4.2 The MC simulation and experimental calculations of the SNR attenuation in Fat tissue. The errors are the standard deviations for 4 data points per slope

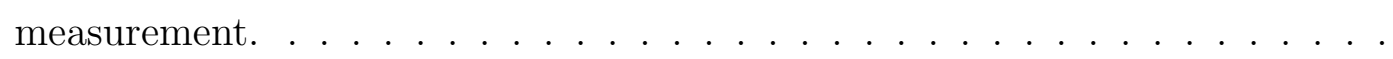

A.1 A list of patient ages and the corresponding measured depths of their left and right bifurcation points using axial MR images at the carotid bifurcation point. The bottom age (N/A) was anonymized and does not reflect the actual age of the patient. . . . . . . . . . . . . . . . .

B.1 The parameter values used in the IAD software. No slides were used during the measurements -the edges of the samples were adhered to the wall of the integrating sphere's sample ports- and as such the thickness is defined as zero. 68 


\section{List of Figures}

1.1 A 3D-constructed MR image using theRadiAnt DICOM viewer $\mathrm{f}$, showing (A)- Right side of the neck surface (B)- Mouth (C)- Common carotid artery (D)- Carotid bifurcation (E)- Internal carotid artery (F)- External Carotid artery. MR images used for reconstruction are courtesy of Dr.General Leung.

1.2 A figure illustration of carotid endarterectomy. (A)- the carotid plaque being removed from inside a branch of the carotid artery and (B)- marking the carotid bifurcation point, a common site of carotid stenosis. Image modified from $[11] \ldots \ldots \ldots \ldots \ldots \ldots \ldots$

1.3 A side-by-side illustrative comparison between stable and vulnerable plaques. Stable plaques share some features with vulnerable plaques, but differ in the severity. The image was reproduced from $[16] . \ldots . . . . . . . . .$.

1.4 The processes of plaque progression into a stable or vulnerable state. Vulnerable plaques can stabilize following a rupture. Image modified from [26]. . .

1.5 A T1-weighted axial MR image showing (Left)- near circumferential IPH. (Right)- Normal Carotid Artery. Image courtesy of Dr. General Leung. Clotting of blood leads to denaturation to the ferric form (MetHb), which leads to the shortening of $\mathrm{T} 1 \mathrm{in} \mathrm{MRI}$ imaging. . . . . . . . . . . . . . . .

1.6 Carotid plaque removed via carotid endarterectomy. Signs of hemorrhaging are visible by the strong presence of metHaemoglobin (marked by arrow). Image courtesy of Dr. General Leung. . . . . . . . . . . . . . . . . . . . 
1.7 A semi-log plot of the Molar Extinction Spectra for the main blood Haemoglobin types: oxyHaemoglobin (OxyHb), deoxyHaemoglobin (DeoxyHb) and metHaemoglobin (MetHb). The distinct features of the spectra suggest the possibility use of optical-absorption contrast for imaging IPH. The plotted spectra are based on the data obtained from $[27,28] \ldots \ldots \ldots \ldots$

1.8 A schematic illustrating the processes behind the Photoacoustic Effect. Modified from [34] . . . . . . . . . . . . . . . . . . . . . 11

1.9 TOP: A semi-log plot of the Molar Extinction Spectra for the main blood Haemoglobin types: oxyHaemoglobin (OxyHb), deoxyHaemoglobin (DeoxyHb) and metHaemoglobin (MetHb). The wavelength of interest is $630 \mathrm{~nm}$, corresponding to the local peak of MetHb, presenting the potential for maximum contrast. BOTTOM: A zoomed-in linear plot of the absorption spectra at expected in-vivo concentrations. . . . . . . . . . . . . . .

2.1 Axial MR image of a patient's neck at the carotid bifurcation point. The arrow marks the depth of the carotid bifurcation point below the skin surface. Image courtesy of Dr. General Leung . . . . . . . . . . . . . . . . . . .

2.2 TOP: The square data point represents the absorption coefficient of muscle tissue [54]. The absorption spectra of the fatty tissue and dermal layers were plotted using reference values in $[35,52,54,55]$. The black line defining the fat(subcutaneous layer + dermis) is a weighted combination of the dermal $(1 / 3)$ and fatty layers $(2 / 3)$. The arrow marks the target "fat" absorption spectra used for simulation and phantom fabrication. BOTTOM: target reduced-scattering spectra for fat and muscle tissues $[52,55]$. . . . . . . . .

2.3 A image illustrating a sample neck tissue model used in the MC simulations and experimental PA measurements. The red lines represent unscattered paths of the incident light for illustration purposes. The image was generated using the maketissue.m Matlab file downloaded from [63] . . . . . . . 
2.4 Image illustrating the setup for creating the base gelatin solution. . . . . . . 26

2.5 Left to Right: completed tissue-mimicking phantoms with 3.1mm-diamter vessels at 25, 20 and $15 \mathrm{~mm}$ depths. All have 10mm thick muscle-mimicking tissue (green) with 15, 10, and 5mm thick skin/fat-mimicking tissue (white) on top. The middle vessel is typically used to inject the metHb; the other two are for redundancy. . . . . . . . . . . . . . . . . . . . 27

2.6 The integrating sphere setup used for the Diffuse Reflectance (left) and Total Transmittance (right) measurements. . . . . . . . . . . . . . . 28

2.7 Phantom samples used for spectrophotometric measurements. A:Fat mimicking, B: Fat absorption (only) mimicking. C: Muscle absorption (only)

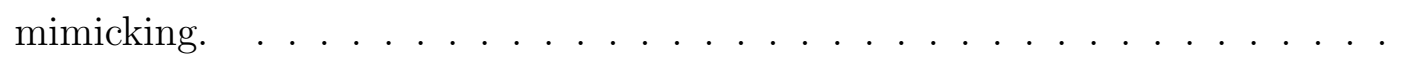

2.8 Fractionated whole blood. The supernatant Plasma layer (C) is separated from the precipitate RBCs layer (A) via a buffy coat (B). . . . . . . . . . . 30

2.9 The experimental setup for PA measurements of the tissue-mimicking phantoms. A - Opotek INC. Radiant tunable laser system. B- L7-4 transducer connected to a Vantage 128 Verasonics system. C- Tissue-mimicking phantom. The red arrows illustrate the direction of the laser beam. . . . . . . . . 31

2.10 An model illustration of the position of the phantom with respect to the laser spot. The phantom is positioned such that the laser spot strikes the vertical mid-point of the vessel, marked by $\mathbf{A}$. Ten measurement trials are completed per phantom for a given MetHb sample, where 3 trials are taken at positions $\mathbf{B}_{\mathbf{1}}$ and $\mathbf{B}_{\mathbf{3}}$, and 4 trials are taken at mid-position marked $\mathbf{B}_{\mathbf{2}} \ldots \ldots$. . . 
2.11 A snapshot showing two individual frames (out of a total of 200 per trial) of the live 2D image view used to guide the free-hand measurement of the PA signals. A- Bottom surface of phantom, i.e. transducer side shown in Figure 2.9. B- The PA signal from the upper boundary of the vessel, i.e. the boundary closer to the laser. C- The PA signal from the fat/muscle boundary. D- Signal from the top surface of the phantom, i.e. the laser side. . . . . . . 33

2.12 A labeled example of an experimentally measured PA RF line. (A)- Location of transducer, (B)- Background noise, (C)- Signal from the lower vessel boundary, (D)- Signal from the upper vessel boundary, (E)- Muscle region, (F)- Signal from the fat/muscle boundary, (G)- Fat region and (H)- Signal from the phantom surface. The SNR per measurement is taken as 20 times the natural logarithm of ratio of the peak signal at the upper boundary of vessel to the standard deviation of background noise: $\mathbf{S N R}=\mathbf{2 0} \log \left(\frac{\mathbf{D}}{\sigma_{\mathrm{B}}}\right) \quad$.

3.1 The measured absorption spectra of human whole blood. Initially, the whole blood (RED) is predominantly oxygenated, as noted by the characteristic OxyHb double peak between $540 \mathrm{~nm}$ and $580 \mathrm{~nm}$. The presence of MetHb $(\mathrm{BLACK})$ can be inferred by the characteristic peak at $\sim 630 \mathrm{~nm}$. The absorption of MetHb at $630 \mathrm{~nm}$ (black dotted line) is approximately 2.3 times greater than that of the oxygenated whole blood (red dotted line). . . . . .

3.2 TOP: Measured average absorption and reduced scattering spectra of the fat phantom layer. The shaded area represents the standard deviation for 14 scattering measurements, and 10 absorption measurements of different phantom samples. Bottom: Measured average absorption and reduced scattering spectra of the muscle phantom layer. The shaded area represents the standard deviation for 5 scattering measurements and 10 absorption measurement of different phantom samples. . . . . . . . . . . . . . . . 
3.3 A photographed imaged of a fabricated $10 \mathrm{~m} 10 \mathrm{f}$ phantom, and the corresponding US B-Mode image on the right. The highlighted regions demonstrate the fat-to-muscle transition on the top, and the $\sim 3.1 \mathrm{~mm}$ wide vessel on the bottom. . . . . . . . . . . . . . . . . . . . . .

3.4 TOP-MC simulation generated 2D light light fluence distribution and the corresponding line plot vs depth at $\mathrm{x}=0 \mathrm{~cm}$ in the $5 \mathrm{~m} 5 \mathrm{f}$ simulation. MIDDLEMC simulation generated 2D light energy absorption distribution and the corresponding line plot vs depth at $\mathrm{x}=0 \mathrm{~cm}$ in the $5 \mathrm{~m} 5 \mathrm{f}$ simulation. BOTTOM$\mathrm{MC}$ generated PA signal amplitude vs. tissue depth at $\mathrm{x}=0 \mathrm{~cm} . \quad \ldots \ldots$

3.5 The MC generated 2D distribution profiles of light fluence (LEFT) and energy deposition (RIGHT) for the 5m5f (TOP) and 5m10f (BOTTOM)

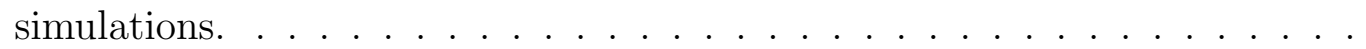

3.6 TOP- Fluence vs. tissue depth, MIDDLE- Energy deposition vs. tissue depth and BOTTOM- Normalized PA amplitude vs. tissue depth for the 5m5f, 5m10f, 5m15f and 5m20f tissue models. All measurements are taken at $\mathrm{x}=0 \mathrm{~cm}$. The solid arrows mark the positions of the top vessel boundary (muscle-to-vessel) where most of the energy is deposited, while the dotted arrows mark the positions of the bottom vessel boundary (vessel-to-muscle) where relatively smaller energy and PA peaks are observed. . . . . . . . . . .

3.7 MC simulated attenuation of peak PA amplitude(dB) normalized to the maximum in $5 \mathrm{~m} 5 \mathrm{f} . \ldots \ldots \ldots \ldots \ldots \ldots \ldots \ldots \ldots \ldots$

3.8 Representative B-mode (2D) PA images in a 10m5f (left) and 5m20f (right) phantoms. A- Signal form MetHb at the vessel boundary. B- Signal from the fat/muscle boundary. C-Signal from the phantom surface. Note the absence of a discernible signal at fat/muscle boundary in the $5 \mathrm{~m} 20 \mathrm{f}$ phantom on the

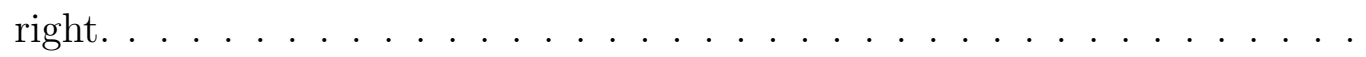


3.9 Representative examples of experimental measurements of PA RF lines in 5m5f (top), 5m10f (middle) and 5m15f (bottom) phantoms with injected metHb samples with absorption coefficients of $90 \mathrm{~cm}^{-1}(\mathrm{RED})$ and $175 \mathrm{~cm}^{-1}$

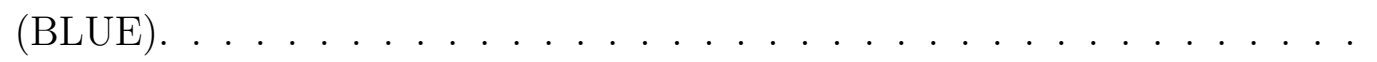

3.10 TOP- Measured SNR(dB) vs. measured MetHb absorption. The red lines define the range of MetHb absorptions in IPH. BOTTOM- Measured SNR(dB) vs. fat thickness for a fixed muscle thickness of $5 \mathrm{~mm}$, with varying injected MetHb absorption strengths. The red line defines the SNR threshold for de-

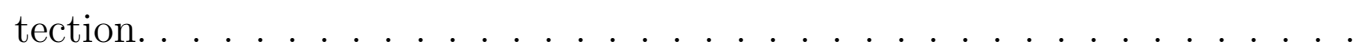

A.1 Axial MR images at the carotid bifurcation point. . . . . . . . . . . 64

A.2 Axial MR images at the carotid bifurcation point. . . . . . . . . . .

B.1 Flowchart for IAD, modified from [67]. The method starts with an initial estimate of the the optical properties to calculate Transmission and Reflection values which are comparing the calculated with the measured values. The process is repeated iteratively with different optical properties until the calculated and measured transmission reflection values match. . . . . . . .

C.1 2D Fluence and Energy deposition profiles for the $5 \mathrm{~m} 15 \mathrm{f}$ simulation. . . . . . 69

C.2 2D Fluence and Energy deposition profiles for the $5 \mathrm{~m} 20 \mathrm{f}$ simulation. . . . . . 70

C.3 2D Fluence and Energy deposition profiles for $10 \mathrm{~m} 5 \mathrm{f}$ simulation. . . . . . . . 71

C.4 2D Fluence and Energy deposition profiles for the $10 \mathrm{~m} 10 \mathrm{f}$ simulation. . . . . 72

C.5 2D Fluence and Energy deposition profiles for the $10 \mathrm{~m} 15 \mathrm{f}$ simulation. . . . . 73

C.6 2D Fluence and Energy deposition profiles for the $10 \mathrm{~m} 20 \mathrm{f}$ simulation. . . . . 74 
D.1 TOP: Measured Total Transmittance spectra (TT)of $8 \% \frac{V_{i}}{V}$ Napthol Green (dye used for muscle absorption) in $10 \%$ Gelatin for one $5.3 \mathrm{~mm}$ (BLACK) and seven $10 \mathrm{~mm}$ (BLUE) phantom samples. These measured spectra, via BeerLambart Law, were used to calculated the absorption spectra of the muscle phantom. MIDDLE: Measured TT spectra of the $\approx 3 \mathrm{~mm}$-thick fat and muscle phantom samples. BOTTOM: Measured Diffuse Reflectance (DR) spectra of the $\approx 3 \mathrm{~mm}$-thick fat and muscle phantom samples. . . . . . . . 76 


\section{List of Symbols, Nomenclature or}

\section{Abbreviations}

IPH : Intraplaque Hemorrhage

MRIPH : Magnetic Resonance Imaged Intraplaque Hemorrhage

PA : Photoacoustic

PAI : Photoacoustic Imaging

TOC : Tissue Optical Clearing

OCA : Optical Clearing Agent

MPE : Maximum Permissible Exposure

CT : Computed Tomography

PET : Positron Emission Tomography

US : Ultrasound

RTE : Radiative Transfer Equation

IAD : Inverse-Adding Doubling

Hb: Haemoglobin

OxyHb: Oxyhaemoglobin

DeoxyHb: Deoxyhaemoglobin

MetHb: Methaemoglobin

MC: Monte Carlo

VIS: Visible

NIR: Near Infrared

VIS-NR: Visible and Near Infrared

IL: Intralipid

NGB: Napthol Green B

FM : Formaldehyde

TT: Total Transmittance

DR: Diffuse Reflectance

WBC: White Blood Cell

RBC: Red Blood Cell

LDL: Low-Density Lipid 


\section{Chapter 1}

\section{Introduction}

\section{$1.1 \quad$ Overview of Strokes}

\subsubsection{Health and Economic Impact of Strokes}

After ischemic heart disease, cerbrovascular events (or strokes) are the leading cause of mortality worldwide, accounting for approximately 7 million deaths or $12 \%$ of all deaths [1]. Strokes are also the second leading cause of disability worldwide [1,2]. Given the costs of of healthcare services, treatment, medication and missed days of work, the annual economic burden of strokes in the European Union, the USA and Canada is over $\$ 40, \$ 60$ and $\$ 3$ billion, respectively [2-4].

Incidence rates of strokes are higher in adults over the age of 65 , with men being at a high risk relative to women [1]. With increasingly aging populations worldwide, the health and economic burdens of strokes continue to rise [5]. The good news about strokes is that survival chances are dramatically increased given early intervention. This creates a need for developing a better understanding of early symptoms and readily accessible and affordable means for preemptive detection of strokes. 


\subsubsection{Strokes: Causes, Risk Factors, and Treatment}

Strokes are of two types, ischemic and haemorrhagic[6]. Haemorrhagic types are less common and occur when blood vessels in the brain rupture and bleed. Ischemic strokes on the other hand account for over $87 \%$ of all strokes and occur when blood flow to the brain is obstructed [6]. The primary underlying cause of obstructions is atherosclerosis caused by plaque and fatty buildup in blood vessels [7]. This results in two types of obstruction events, thrombotic and embolic [8]. Thrombotic events occur when a blood clot known as a thrombus forms in an artery in the brain. The more common embolic events occur when a blood clot/debris known as an emobolus forms away from the brain, and then travels to the brain causing an obstruction [8]. One of the primary suspects of the latter type is carotid stenosis, brought by atherosclerosis of the carotid artery [7]. As seen in Figure 1.1, the carotid artery starts from below the neck a singular vessel known as the common cartoid artery, which then splits into the internal and external carotid arteries at the bifurcation point. The carotid bifurcation point is the most common site of carotid stenosis [9].
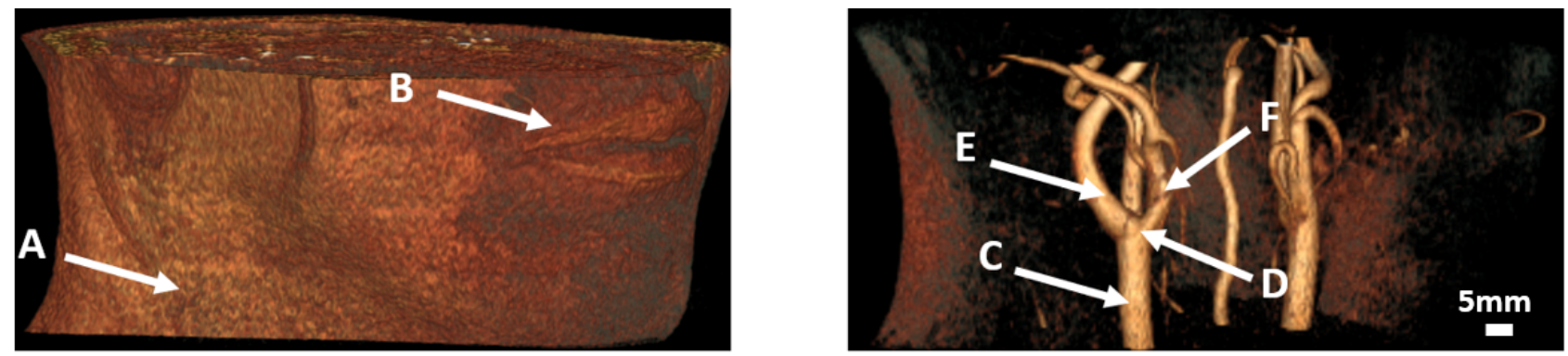

Figure 1.1: A 3D-constructed MR image using theRadiAnt DICOM viewer f, showing (A)Right side of the neck surface (B)- Mouth (C)- Common carotid artery (D)- Carotid bifurcation (E)- Internal carotid artery (F)- External Carotid artery. MR images used for reconstruction are courtesy of Dr.General Leung.

Several modifiable and unmodifiable factors contribute to an increased risk of strokes. Given the vascular roots of strokes, the common modifiable risk factors related to cardiovascular disease apply to strokes. These include, but are not limited to hypertension, smoking, diabetes, high cholesterol imbalance, obesity and lack of physical activity [10]. Some unmodifiable risk 
factors include sex, age, and genetics; people suffering from sickle-cell anemia, a disease more prevelant withing the black and Hispanic peoples, are at a higher risk of ischemic strokes[10].

Beside lifestyle changes, prevention and treatments of strokes involve either pharmaceutical or surgical interventions. Pharmaceutical intervention makes use of blood-thinners such as anticoagulants and antiplatelets to to reduce the risk of blood clots, as well as antihypertensives to treat hypertension[11]. Surgical interventions include carotid endarterectomy, which involves the surgical removal of a blood vessel blockage (plaque) from the carotid artery. Another surgical measure uses angioplasty or stents, wherein a doctor forcefully opens a blocked blood vessel by using balloon angioplasty and implantable steel screens called stents [11]. The degree of stenosis and vulnerability of plaque dictate the severity of intervention necessary.

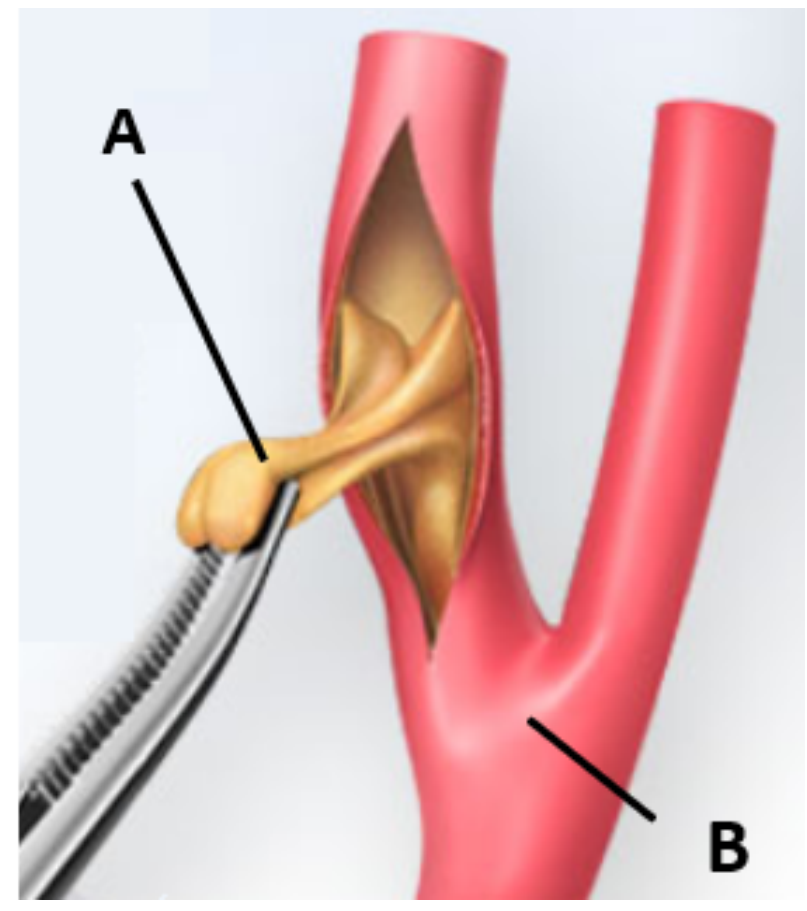

Figure 1.2: A figure illustration of carotid endarterectomy. (A)- the carotid plaque being removed from inside a branch of the carotid artery and (B)- marking the carotid bifurcation point, a common site of carotid stenosis. Image modified from [11]. 


\subsubsection{Carotid Plaque Vulnerability}

Carotid plaques are not uncommon in healthy persons. Carotid plaques are typically categorized as either stable or unstable (vulnerable) [12], with the former carrying a relatively low risk factor for cerebrovascular events $[12,13]$. One telling sign of a patient's vulnerability to cerebrovascular events is the vulnerability of the carotid plaque. Stable plaques are often asymptomatic and carry little risk [13,14]. Vulnerable plaques, on the other hand are intimately linked to a higher risk of ischemic events[13]. Distinguishing stable from unstable plaques is therefore important, as each requires different intervention.

\subsubsection{The Process of Arteriosclerosis}

Arteriosclerosis of the carotid starts with an accumulation of excess low-density lipids (LDLs) in the arterial wall, forming the plaque's lipid core [15]. The LDLs undergo oxidation, causing chemical alterations that trigger an inflammatory response [15]. This leads to an influx of white blood cells (WBCs) [15]. The byproducts of inflammation lead to further growth and progression of the plaque by forming a fibrous cap with microvasculature around the lipid core. This leads to a thickening of the plaque, which narrows of the arterial lumen [15]. Further inflammatory substances can lead to weakening of fibrous cap, making it vulnerable to rupture and possibly lead to intraplaque haemorrhaging (IPH) as well $[15,16]$.

\subsubsection{Stable vs. Unstable Plaques}

Figures 1.3 and 1.4 illustrate the differences between a stable and vulnerable plaque, and the progress from a normal artery to either a stable or unstable plaque. The typical histological markers of plaque vulnerability include plaque inflammation with monocytes, macrophage, and T-lymphocyte infiltration, a thin fibrous cap with large lipid core, endothelial denudation with superficial lipid aggregation, fissured plaque, $>90 \%$ stenosis, superficial 
calcification and intraplaque haemorrhage (IPH) $[17,18]$. While a stable and unstable plaque share some features, they different in the severity and magnitude, as shown in Figure 1.3. Compared to vulnerable plaques, stable plaques have smaller lipid cores, thicker fibrous caps, lower WBC -or macrophage- content, less microvasculature, no IPH or rupture and macroinstead of micro-calcification [19]. It is observed in Figure $\mathbf{1 . 4}$ that an unstable plaque can stabilize following a rupture.

While vulnerable plaques might not necessarily exhibit all the features mentioned, some features such as the IPH, are a uniquely independent risk factor.

\subsubsection{IPH and Recurrent Strokes}

IPH is caused by the rupturing of blood vessels invading atherosclerotic plaques. It is a common feature of atherosclerotic plaques, and is considered one of the identifying features of lesions preceding ischemic events [18,20-23]. Beside being a critical destabilizing factor in carotid plaques, IPH-positive plaques carry strong predictive power for repeat strokes [21]. The presence of IPH strongly predicts repeated ischemic events for symptomatic $\geq 50 \%$ carotid stenosis [22]. The risk difference between those with and without IPH for recurrent stroke was shown to be $+14.9 \%,+50.9 \%$, and $+66.8 \%$ at years 1,3 , and 5 , respectively [21]. Moreover, IPH is predictor of negative outcomes in post-operative patients, and is even believed to be an important contributing factor in patients suffering from embolic strokes of an undetermined source (ESUS) [20].

The strong association between IPH and recurrent ischemic events, makes it a strong independent predictor of risk of recurrent ischemic events in symptomatic carotid patients. This assists in better patient selection for invasive interventions, reducing the risk-benefit ratio. With approximately one in four of all stroke cases being recurrent [21], a need rises for a safe and accurate means of detecting IPH in carotid plaques. 


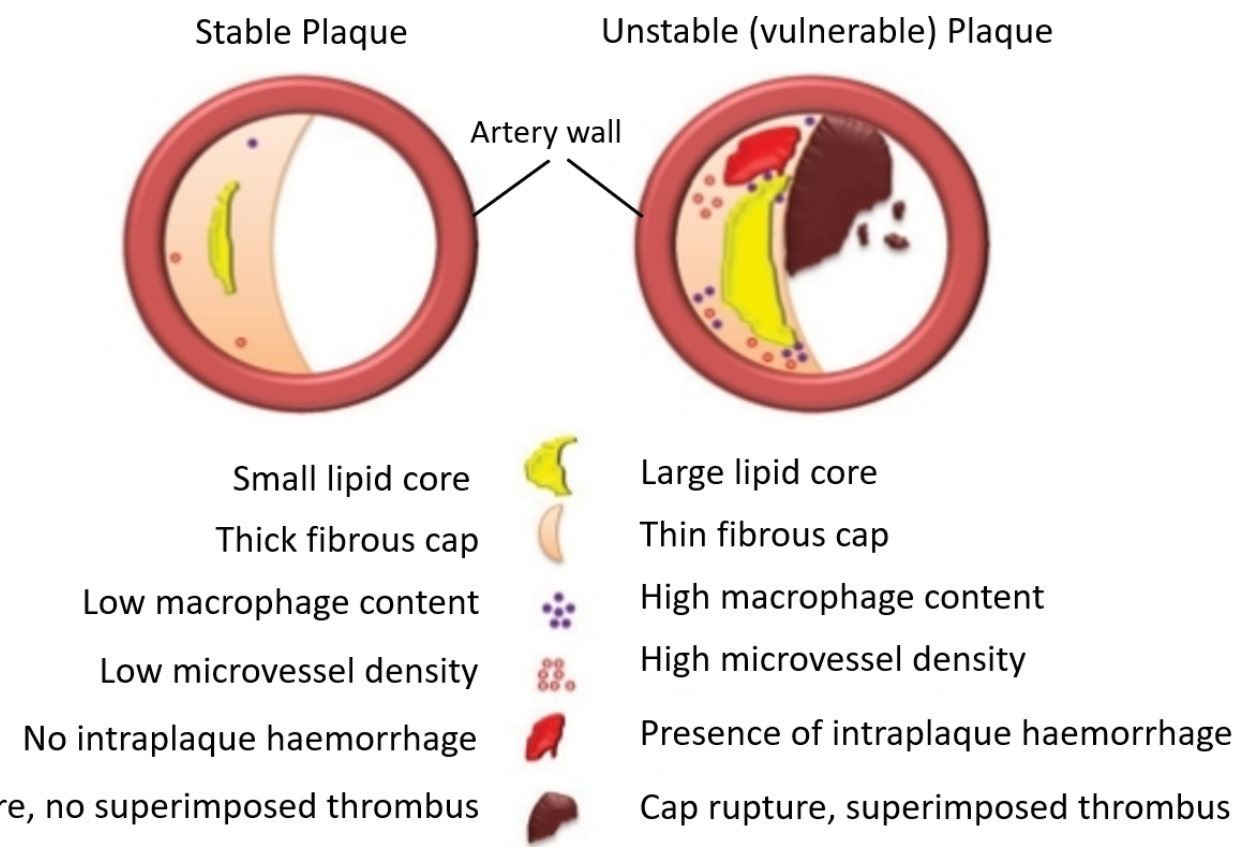

Figure 1.3: A side-by-side illustrative comparison between stable and vulnerable plaques. Stable plaques share some features with vulnerable plaques, but differ in the severity. The image was reproduced from [16].

\subsubsection{Non-invasive Imaging of Vulnerable Plaques and IPH}

The common non-invasive techniques used to image for and detect plaque vulnerability are computed tomography (CT), ultrasound imaging (US), magnetic resonance imaging (MRI), and positron emission tomography (PET) $[17,25]$. While US imaging is the most readily available low-risk tool, it lacks contrast to reliably detect individual plaque components[17,23]. On the other hand, CT scans are considered the best for identifying plaque calcification, while PET scans are useful in assessing metabolic functions, given their use of radioisotope tracers. PET cans do however suffer from limited spatial resolution resulting in poor anatomic delineation. MRI holds the most potential, with good sensitivity and specificity for most plaque features $[17,25]$.

Pathology studies have been linking IPH to strokes for over 40 years. It is only in the past 15-20 years, however, that a method for noninvasviely detecting IPH was developed. MRI is currently the only imaging modality capable of noninvasively detecting IPH $[17,22,25]$. Other 


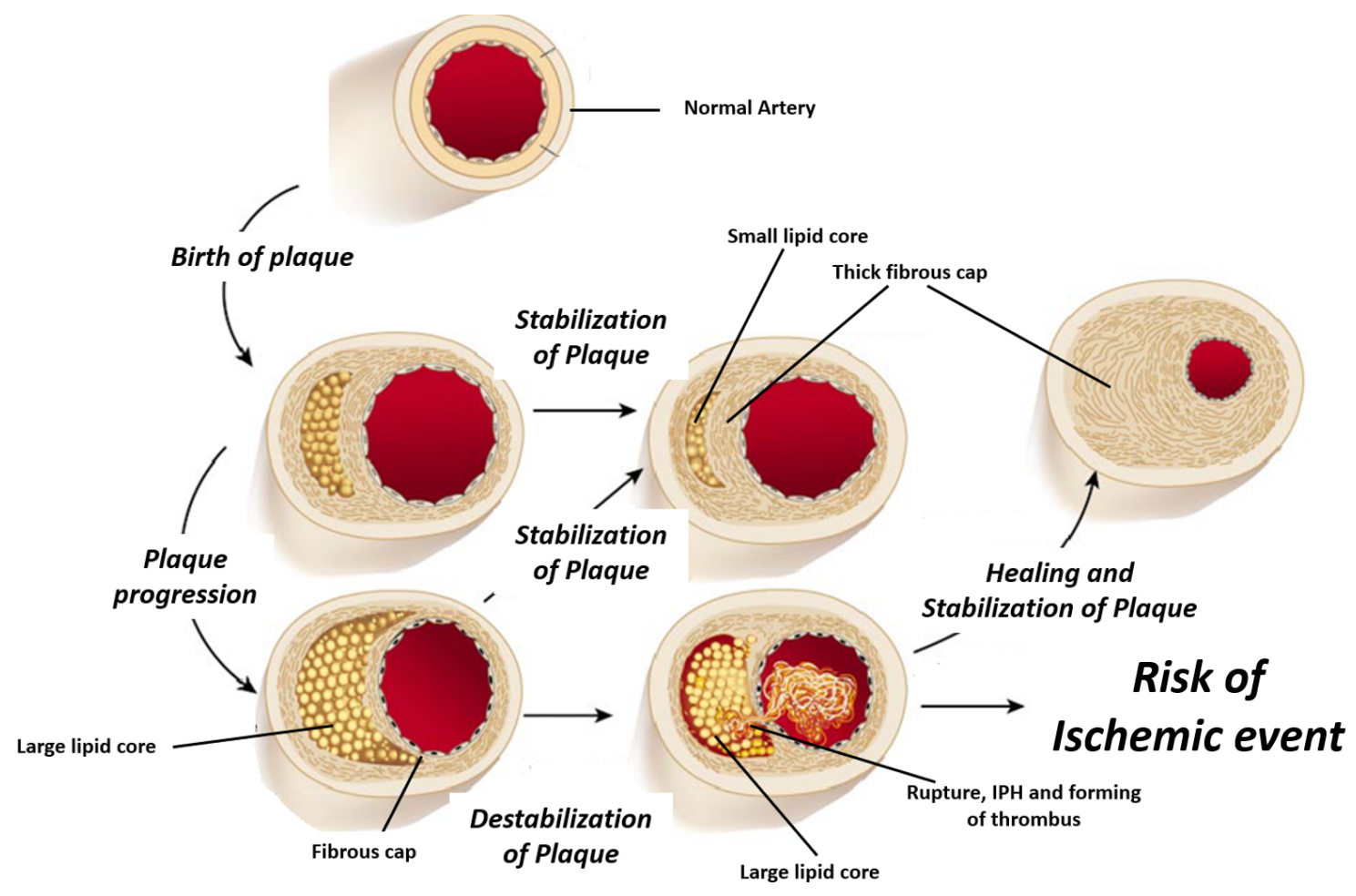

Figure 1.4: The processes of plaque progression into a stable or vulnerable state. Vulnerable plaques can stabilize following a rupture. Image modified from [26].

modalities lack the necessary contrast for discriminating IPH [17,24]. The contrast in MR images of IPH (or MRIPH), as shown in Figure 1.5 is due to endogenous methaemoglobin [27]. The presence of methaemoglobin can bey verified in plaque samples removed by endarterectomy, as shown in Figure 1.6.

\subsubsection{An Alternative to MRI}

The strong presence of endogenous MetHb in IPH suggests optically based approaches for imaging, capitalizing on the distinct optical features of MetHb. MetHb has a unique absorption spectra in the visible (VIS) range as seen in Figure 1.7. Using purely optical imaging however is not feasible given strong the tissue attenuation of light as carotid arteries are typically over a centimeter deep under the skin. An imaging technique based on the same optical contrast that manages to overcome the limit of purely optical techniques is Photoa- 


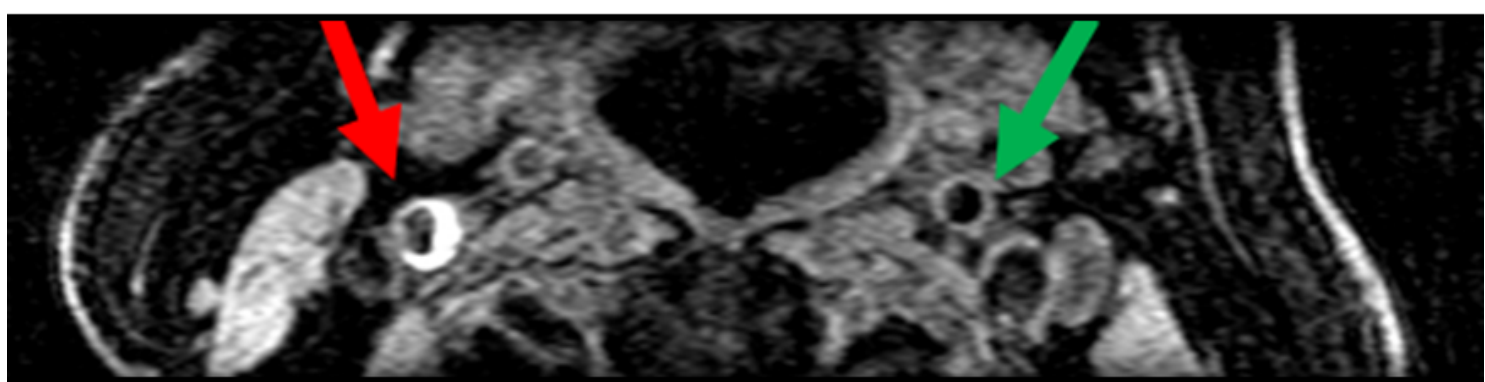

Figure 1.5: A T1-weighted axial MR image showing (Left)- near circumferential IPH. (Right)- Normal Carotid Artery. Image courtesy of Dr. General Leung. Clotting of blood leads to denaturation to the ferric form (MetHb), which leads to the shortening of $\mathrm{T} 1$ in MRI imaging.

coustic Imaging (PAI). PAI has the potential to offer a less costly and more readily accessible alternative to MRI for the imaging and detection of IPH.

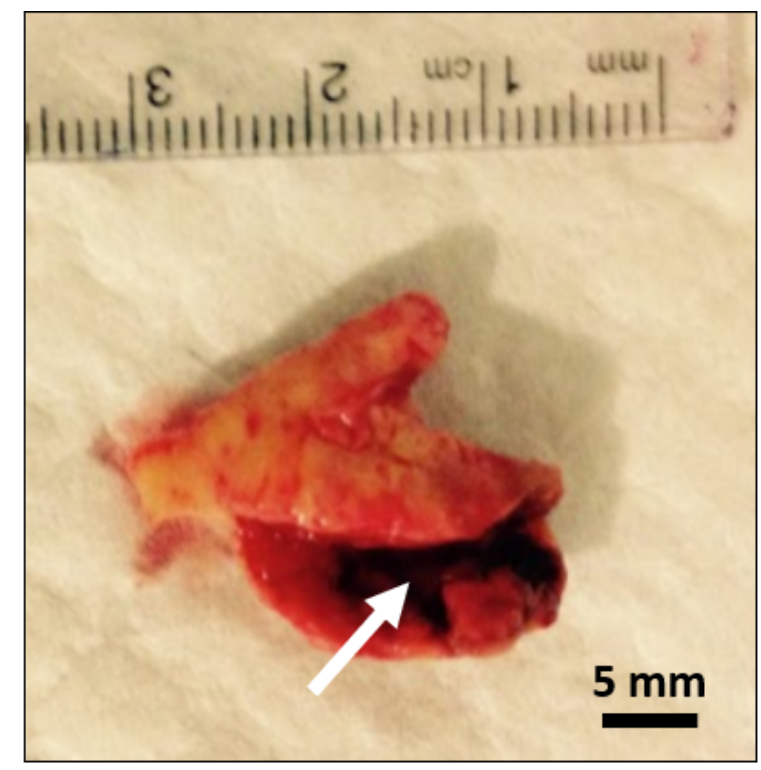

Figure 1.6: Carotid plaque removed via carotid endarterectomy. Signs of hemorrhaging are visible by the strong presence of metHaemoglobin (marked by arrow). Image courtesy of Dr. General Leung. 


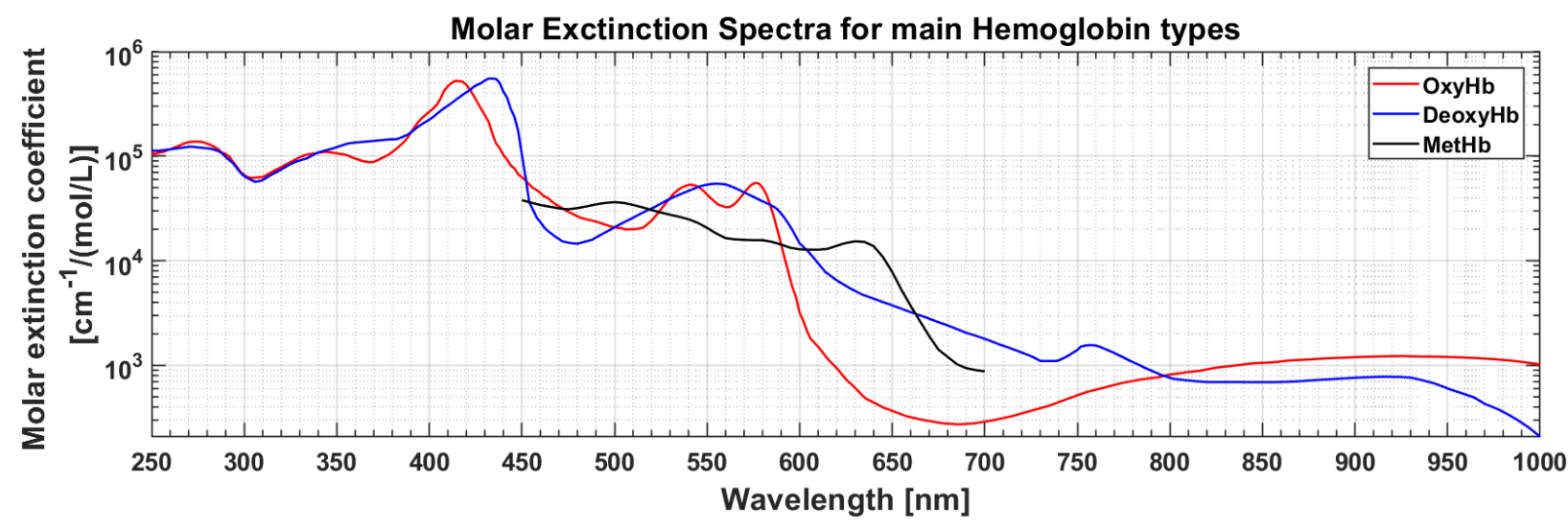

Figure 1.7: A semi-log plot of the Molar Extinction Spectra for the main blood Haemoglobin types: oxyHaemoglobin (OxyHb), deoxyHaemoglobin (DeoxyHb) and metHaemoglobin (MetHb). The distinct features of the spectra suggest the possibility use of optical-absorption contrast for imaging IPH. The plotted spectra are based on the data obtained from $[27,28]$.

\section{$1.2 \quad$ Principles of Photoacoustic Imaging}

\subsubsection{Photoacoustic Effect}

The Photoacoustic (PA) effect is the physical basis for photoacoustic imaging. It refers to the mechanism by which acoustic waves are generated upon the absorption of EM energy in a sample material. Ever since the discovery of the PA effect in 1880 by Alexander Graham Bell [29] in 1880, PA applications have come a long way in the fields of physics, chemistry and biology and medicine [31-33].

Photoacoustic signal generation can be described in three steps. First, a chromophore aborbs EM radiation, such as light. The absorbed EM energy is then converted into heat, which generates a temperature rise, typically in the milli-kelvin range. Finally, thermoelastic expansion takes place, which results in the emission of pressure (acoustic) waves. Figure 1.8 shows an illustrative schematic of the whole process.

In order for acoustic waves to be generated, the optical illumination must be time variant[30].

This is can be achieved by using either a pulsed laser or a intensity modulated continuous- 
wave laser. Pulsed excitations are more commonly used as they produce higher SNR than continuous-wave excitations [31]. Following a short laser pulse excitation, the local fractional volume expansion can be expressed as [31,32]:

$$
\frac{d V}{V}=-\kappa p(\vec{r})+\beta T(\vec{r})
$$

where $\kappa$ is the isothermal compressibility, $\beta$ is the thermal coefficient of volume expansion, and $p(\vec{r})$ and $T(\vec{r})$ are changes in pressure and temperature, respectively.

For effective PAT signal generation, the laser pulse duration is kept below the thermal and stress confinement times [31,32]. The thermal confinement condition ensures that that thermal diffusion during laser illumination can be neglected:

$$
\tau<\tau_{t h}=\frac{d_{c}^{2}}{4 D_{T}}
$$

where $\tau_{t h}$ is the thermal confinement threshold, $d_{c}$ is the dimension of the optical absorber, and $D_{T}$ is the thermal diffusivity for soft tissue, which is approximately $0.14 \mathrm{~mm}^{2} / \mathrm{s}$.

The stress confinement condition ensures that the volume expansion of the absorber during the illumination period can be neglected:

$$
\tau<\tau_{s t}=\frac{d_{c}}{v_{s}}
$$

where $v_{s}$ is the speed of sound within the optical absorber.

If both thermal and stress confinement conditions are satisfied, the absorbed energy creates an initial photoacoustic pressure rise estimated by $[31,32]$ :

$$
p_{0}=\frac{\beta T(\vec{r})}{\kappa}
$$


The temperature rise $T$ can be further expressed as a function of optical absorption:

$$
T=\frac{A_{e}}{\rho C_{V}}
$$

with $\rho$ the mass density, $C_{V}$ the specific heat capacity at constant volume, and $e_{e}$ the specific absorbed energy density $\left(\mathrm{J} / \mathrm{m}^{3}\right)$, which is a product of the absorption coefficient $\mu_{a}$ and the local optical fluence $F(\vec{r})$.

Combining equations 1.4 and 1.5 , we can write the initial photoacoustic pressure as [31,32]:

$$
p_{0}=\frac{\beta A_{e}}{\rho C_{V} \kappa}=\Gamma \mu_{a} F(\vec{r})
$$

where $\Gamma=\frac{\beta}{\rho C_{V} \kappa}$ denotes the Grueneisen parameter, a temperature-dependent physical characteristic that characterizes the thermoelastic efficiency of materials.

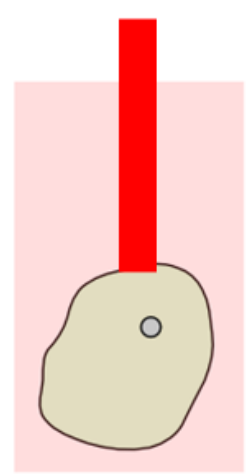

Pulsed EM (laser) irradiation

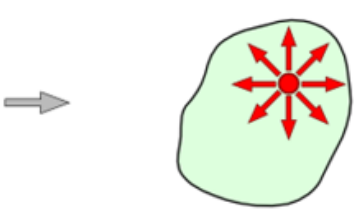

Optical absorption \& thermoelastic expansion

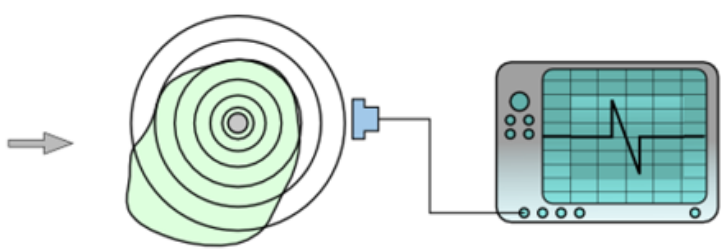

Ultrasonic wave generation and detection

Figure 1.8: A schematic illustrating the processes behind the Photoacoustic Effect. Modified from [34]. 


\subsubsection{Why Photoacoustic Imaging?}

Purely optical imaging modalities suffer from limited resolution and penetration depth due to high optical attenuation in biological tissue, limiting its use to low-diffusion regimes of superficial depths $<1 \mathrm{~mm}$ [32-34]. The modalities however, offer the potential of enhanced contrast due to the drastically different optical properties, particularly absorption, of different biological components. On the other hand, ultrasonic scattering is 2 to 3 orders of magnitude smaller than optical scattering, allowing imaging at higher resolution for depths beyond $1 \mathrm{~mm}$ [32-34]. Ultrasound imaging however does suffer from relatively poor contrast, as it is dependent on the mechanical properties of tissue, making it unsuitable for detecting subtle physiological or biochemical changes [32-34]. Photoacoustic imaging is based on both modalities while overcoming their shortcomings. One can indeed think of Photoacoustic Imaging as contrast-enhanced ultrasound imaging.

Similar to ultrasound imaging, the spatial resolution of PA imaging is limited by the bandwidth of the PA waves generated. A PA signal with a frequency of $1 \mathrm{MHz}$, for instance, can provide approximately a $1.5 \mathrm{~mm}$ spatial resolution, given that the average speed of sound in soft tissues $\approx$ is $1500 \mathrm{~m} / \mathrm{s}$. Higher frequencies can provide higher better resolution, but at the expense of higher acoustic attenuation. PA signals can be detected using regular ultrasonic transducers, whether linear, curved or tomographic [31-33].

\subsubsection{Light interactions in tissue}

The ability of EM waves to penetrate tissue and deposit its energy is crucial in Photoacoustic Imaging. This requires the characterization of optical properties of biological tissues. Given the very high absorption of water in the Terahertz range, the faesbile window for PA falls within the near-infrared and visible or NIR-VIS range of $400 \mathrm{~nm}$ to $1200 \mathrm{~nm}$, otherwise known as the optical window [31]. 
Light undergoes one of four interactions in biological tissues: transmission, absorption, reflection or scattering. Transmission is the absence of interactions, or forward elastic scattering of light wherein the light carries on the same path unattenuated. Reflection is defined as either diffuse or specular (mirror-like), and is a consequence of a mismatch in the refractive index of materials light travels through. Broadly speaking, reflection can be considered as a type of scattering. Approximately $4 \%$ to $7 \%$ of incident light is reflected off the skin, with less light being reflected at a lower incident angle [35]. Absorption and scattering are the most dominant and photoacoustically relevant types of light interactions in biological tissue.

Absorption refers to the mechanism by which the energy of a photon is absorbed by matter. Molecules that absorb light are called chromophores. The main absorbers in biological tissue within the NIR-VIS range are water, haemoglobin, melanin and lipids [36]. Scattering refers to the mechanisms by which light deviates from its straight path due to non-uniformities, such as mismatch in refractive index, in matter. Mitchondria and collagen fibers in tissue are two of the primary sources of light scattering in biological tissue [36].

\subsubsection{Biomedical Applications of Photoacoustics}

Given an appropriate ultrasound transducer bandwidth for detection, PAI enables the imaging of a wide array of sizes, ranging from organelles to organs [37], and with proper choice of single, or even multiple laser wavelengths, PA is capable of generating a wider array optical contrast due to the changes in optical absorption of different biological components with changing wavelengths.

One of the most common targets of PAI is Haemoglobin. This makes biological imaging targets heavily dependent on haemoglobin, such as cancerous tumors, a common target on PA imaging [38-40]. Hypoxic areas are one of the hallmarks features of tumors, as hypoxia is an important factor of tumor growth and angiogenesis (growth of new blood vessels)[38-40]. 
To sustain the growth of tumors, new oxygen and food must be supplied, forcing the formation of complex vascularization. The growth and malignancy of the tumor can be therefore determined based on the microvasculature structure and the oxygen saturation of the tumor. PAI can be used to do so given the unique optical signature of hemoglobin in the NIR region. The use of PAI goes beyond just the detection of tumors, as it can also provide timely evidence on the efficacy of treatments by detecting local post-treatment changes much earlier and much shorter time scales than in other non-invasive modalities [41,42]. Other clinical applications of PAI include the detection of red blood cell aggregation in diabetes, US-PA dual detection of study of human inflammatory arthritis, and US-PA dual interventional imaging of placentas for minimally invasive fetal surgeries [43-45].

PAI also demonstrates potential for imaging carotid arteries for detecting carotid artery disease. Using phantoms and in-vivo studies, Dima et al demonstrate the feasibility of detecting carotid in clinically relevant depths using Multi-spectral PA tomography [45]. They conclude that by using tomographic approaches with curved arrays, stronger SNR can be achieved as compared to traditional linear-array US transducers. Jansen et al demonstrated the use of intravascular PAI (IVPA) of carotid plaques using ex-vivo human specimens, while Wang et al performed IVPA for imaging carotid plaques in-vivo in rabbits $[47,48]$. The above attempts all focused primarily on detecting lipid-rich plaques as means for contrast. Alternatively, Arabul et al used ex-vivo PAI imaging of carotid endarterectomy plaque samples to confirm the presence of IPH that was otherwise invisible using US imaging [49]. There is no literature on the use of non-invasive use of PAI for detecting IPH, however. A more comprehensive review of recent progress in the overall PAI of atherosclerosis and vulnerable plaques is found in [50].

The two main obstacles limiting the wider usage of PAI, particularity for deeper tissue imaging, is the attenuation of light and weak target chromophore absorption. Techniques 

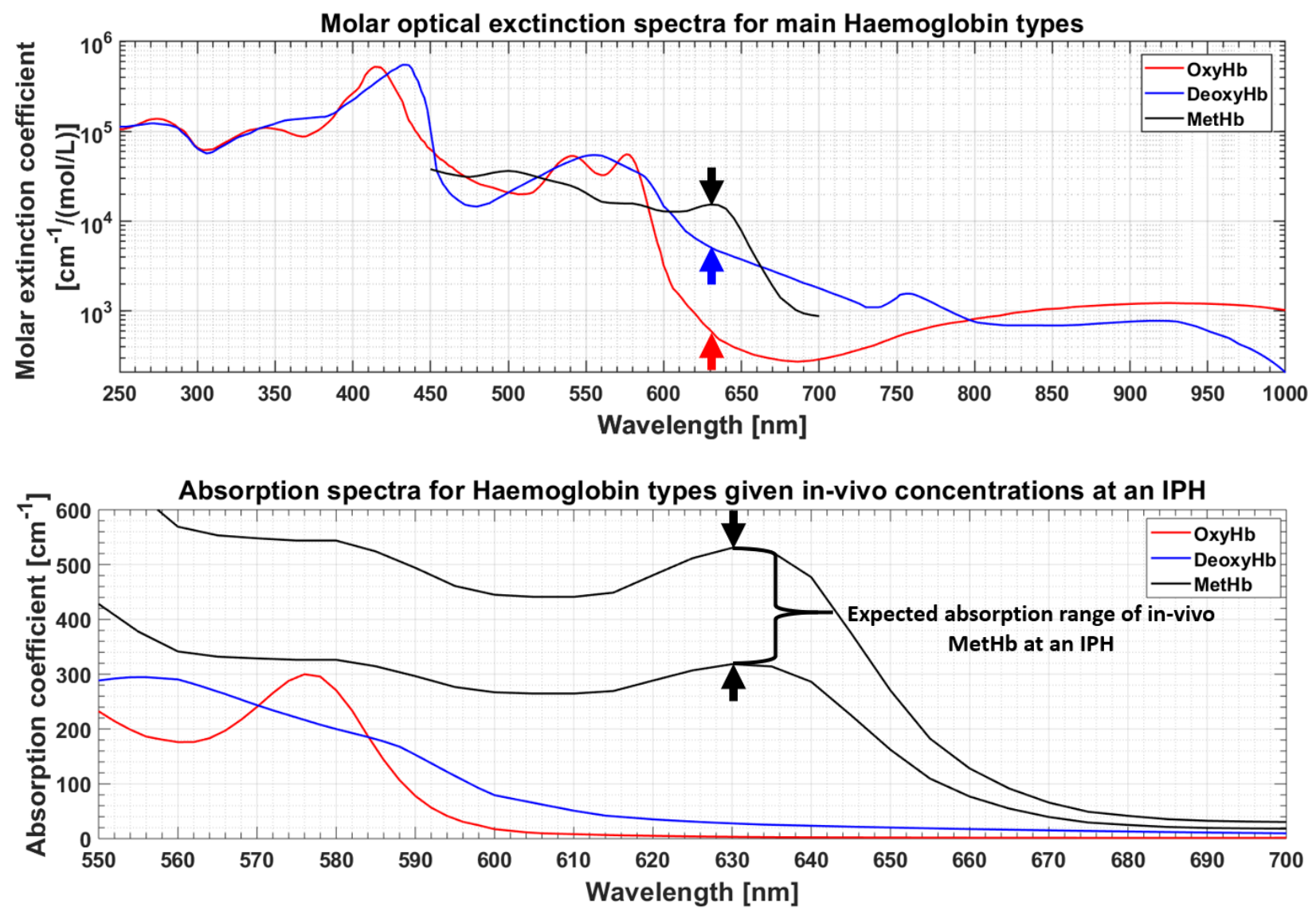

Figure 1.9: TOP: A semi-log plot of the Molar Extinction Spectra for the main blood Haemoglobin types: oxyHaemoglobin (OxyHb), deoxyHaemoglobin (DeoxyHb) and metHaemoglobin (MetHb). The wavelength of interest is $630 \mathrm{~nm}$, corresponding to the local peak of MetHb, presenting the potential for maximum contrast. BOTTOM: A zoomed-in linear plot of the absorption spectra at expected in-vivo concentrations.

such as tissue optical clearing (TOC) can potentially be used to improve the former, whereby topical or superficially injected clearing agents are used to reduce the optical scattering of top layer of skin [51]. Exogenous contrast agents such as gold nano-particles can be used to artificially enhance target absorption, and in doing so, improve contrast [52].

\subsection{Motivation, Hypothesis and Specific Aims}

MRI is presently the only way to noninvasively image and detect carotid IPH. In addition to being costly, access to MRI scanners can be limited in remote or rural areas which resources. MRI scanners are not as widely available in smaller clinics as X-Ray or Ultrasound machines. 
This is particularly relevant given the higher of stroke in developing countries, where access to advanced imaging machines is restricted [1].

The motivation behind this thesis is to investigate a readily accessible and economical method of non-invasively imaging carotid IPHs: PAI can be incorporated onto US machines. Fujifilms's Vevo LAZR systems, for instance, operate as hybrid US/PAI systems. This gives PAI systems the potential benefit of mobility and accessibility that current US systems enjoy. The end goal is to eventually develop an accessible and more economical means of earlier non-invasive detection of carotid IPH, which would help the patient and concerned physician(s) to take earlier preemptive measures against potential future stroke by detecting IPH in carotid plaques. We see PAI of carotid IPH being a an important component in carotid plaque imaging.

The imaging of carotid plaques in-vivo has been shown to be feasible [46-50]. However, current attempts of carotid plaques are either invasive (e.g intravascular) and/or target lipidrich cores in carotid plaques instead of IPH for contrast in carotid plaque. There currently is no literature on the non-invasive imaging of carotid IPH in-vivo. In this light, the the hypothesis guiding this thesis is that PA techniques can be used for the non-invasive imaging and detection of carotid IPH, and to that end, this thesis addresses three main specific aims:

1. Determine the relevant tissue types and associated optical absorption and scattering of the human neck. This will guide towards the creation of a neck tissue model to be used for theoretical (Monte Carlo) and experimental studies.

2. Conduct Monte Carlo simulation of light propagation, energy absorption and photoacoustic signal generation from MetHb in tissue mimicking models. This is carried out with varying depths (or tissue thicknesses) to study the SNR with increasing depth.

3. Conduct experimental measurements of PA signals generated from MetHb in tissue- 
mimicking phantoms. This will be carried out using different MetHb concentrations and tissue depths to study SNR with MetHb absorption and SNR with depth to estimate the expected maximum imaging depth for carotid IPH detection.

\subsection{Thesis Outline}

This thesis is organized into five chapters. The first chapter starts with an introduction of the health and economical impacts of strokes, types of strokes, vulnerable plaques, IPHs and non-invasive techniques for imaging strokes and IPH. It also presents an overview of PAI, the physics behind technique, limitations and and medical applications thereof. Chapter 2 describes the tools, methods and procedures followed in achieving the specific aims. Chapter 3 presents the results relevant to the specific aims, while Chapter 4 includes the discussion,

interpretation and relevance of results. Finally, Chapter 5 presents the thesis summary, conclusions and future work. 


\section{Chapter 2}

\section{Methods and Tools}

This section describes the methods, procedures and resources used in : (1) determining the neck tissue types and their relevant optical properties. (2) the Monte Carlo simulation of optical prorogation in tissue models, (3) tissue phantom fabrication and measurement of optical properties, and (4) experimental Photoacoustic measurements of tissue-mimicking phantoms.

\subsection{Determining Tissue Properties}

\subsubsection{Neck Tissues}

In order to create accurate tissue models for theoretical and experimental studies, the physical and optical properties of the neck must be first established. MR images were used to determine the average depth of the carotid bifurcation point. Published literature was used to determine the thicknesses of relevant tissue types and their optical absorption and scattering. The primary tissue types making up the human neck - to the carotid- are the skin and sternocleidomastoid muscle. Skin is typically divided into its three constituents layers, with the epidermis on the top, then the dermis and hypodermis. The hypodermis is often treated separately and called subcutaneous tissue. Sixteen patients between the ages of 46 
and 115 years, with a median age of 65 years, had an MR image of the neck taken. The axial plane at the bifurcation point, similar to Figure 2.1 was used to estimate the depth of both the right and left carotid bifurcations. The depths ranged between $9 \mathrm{~mm}$ and $31 \mathrm{~mm}$ averaging at $(21.3 \pm 5.4) \mathrm{mm}$. A table listing the depths as well as a sample of used MR images of can be found in Appendix A.

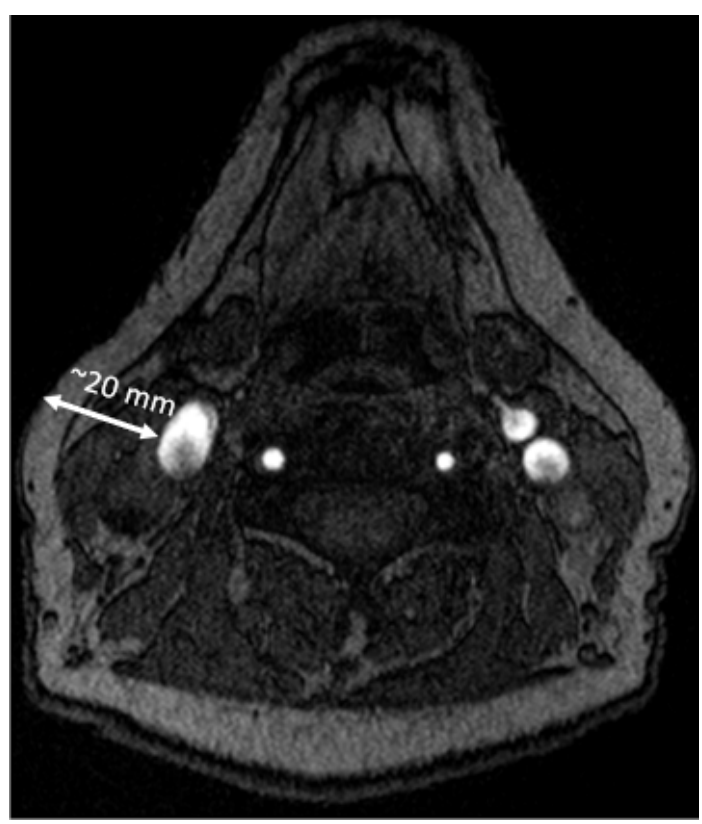

Figure 2.1: Axial MR image of a patient's neck at the carotid bifurcation point. The arrow marks the depth of the carotid bifurcation point below the skin surface. Image courtesy of Dr. General Leung

Epidermal and dermal thicknesses range from 60 to $100 \mu \mathrm{m}$ and 2 to $4 \mathrm{~mm}$, respectively $[49,50]$. The thickness of the subcutaneous layer shows more variance from to person, especially between thin and obese patients. Based on the MR images studied, the subcutaneous layer thickness ranged between between 5 and $20 \mathrm{~mm}$. The muscle thickness ranged between 5 and $10 \mathrm{~mm}$. While the skin is multi-layered, the dermal layer is combined with the subcutaneous to create a singular "fat" layer; unless otherwise stated, all reference to "fat" relating to phantom and tissue models implies a combination of both the dermis and subcutaneous layers. The epidermal layer, typically less than a millimeter thick, and for the 
sake of phantom fabrication practicality, is ignored in the phantom making process. The epidermis does have a disproportionately strong absorption, however, an will hence have contribution is corrected for in the discussions chapter. Figure 2.2 shows the absorption and reduced scattering spectra of fat and muscle tissues, as modified from [36,53,55,56]. All tissue types are taken to have an anisotropy factor of $\mathrm{g}=0.9[35,57]$.

A bi-layer rectangular cube tissue-mimicking model is proposed for both numerical and experimental measurements; a top layer defining the "fat" and a bottom layer defining the muscle. $\mathrm{A} \sim 3.1 \mathrm{~mm}$ diameter vessel representing a carotid lumen is positioned at the center below the muscle layer. Table 2.1 shows the dimensions and optical properties of the proposed tissue models. The absorption of "fat" was determined by a weighed combination of the dermal and fatty layer absorptions. Given the thicknesses of the "fat" layer of 5,10,15 and 20 , the average fraction a dermis constitutes is $\sim 30 \%$, giving it a weight of 0.3 and the subcutaneous fatty tissue a weight of 0.7 .

The tissue types discussed above describe the path up to the carotid artery wall. Given that the plaque and IPH would be located on the lumen's side of the wall, the carotid wall contributes to the attenuation of light arriving at the IPH. For practicality, the wall and epidermis are ignored in the simulation and the phantom fabrication processes. Their properties and contribution are discussed in Chapter 4, however.

\subsubsection{Plaque and IPH}

The average thickness of high-risk carotid plaque is $2.7 \mathrm{~mm}$, with its components being WBCs (macrophages), lipids, a fibrous cap, calcification (hydroxyapatite), IPH and microvascula-

ture [47,58-62]. The fibrous cap consists primarily connective tissue made up of elastin and collagen, covering the lipid core from the blood flow in the carotid lumen above it. 
At $630 \mathrm{~nm}$, the optical absorption coefficients of lipids, collagen, elastin and hydroxyapatite are $\sim 0.008 \mathrm{~cm}^{-1}, \sim 0.08 \mathrm{~cm}^{-1}, \sim 0.25 \mathrm{~cm}^{-1}$ and $\sim 0.01 \mathrm{~cm}^{-1}$ respectively, with macrophages having negligible absorption $[47,59,60,62]$. The microvasculature is assumed to carry primarily oxygenated blood (OxyHb) which has an absorption coefficient of $\sim 3-4 \mathrm{~cm}^{-1}$ at 630nm. The absorption of IPH is primarily due to MetHb, which is expected to be between $310 \mathrm{~cm}^{-1}$ and $518 \mathrm{~cm}^{-1}$.

Given the lower absorption of non-IPH plaque components at 630nm, the proposed model for an plaque with IPH ignores non-IPH components and assumes a vessel filled entirely with MetHb. Due to the strong absorption and optical attenuation of MetHb, it is expected that most of the energy absorption, and consequently PA generation, would occur at the boundary of the vessel, with weak to minimal contribution from regions deeper in the vessel. As such, the optical effects of the blood in the lumen is ignored. This, however, does assume that the plaque (and IPH) are located at the artery wall nearer to the light source rather than the wall further away. If the latter was the case, the blood in the lumen would act as an additional attenuator of light before reaching the IPH.

\begin{tabular}{|l|c|c|}
\hline & Fat & Muscle \\
\hline Thickness $(\mathrm{mm})$ & $5,10,15,20$ & 5,10 \\
\hline Absorption coefficient $\left(\mathrm{cm}^{-1}\right)$ & 0.15 & 1.2 \\
\hline Reduced Scattering coefficient $\left(\mathrm{cm}^{-1}\right)$ & 10.0 & 8.0 \\
\hline
\end{tabular}

Table 2.1: Dimensions and optical properties at $630 \mathrm{~nm}$ of the proposed tissue-mimicking $\operatorname{model}(\mathrm{s})$. 

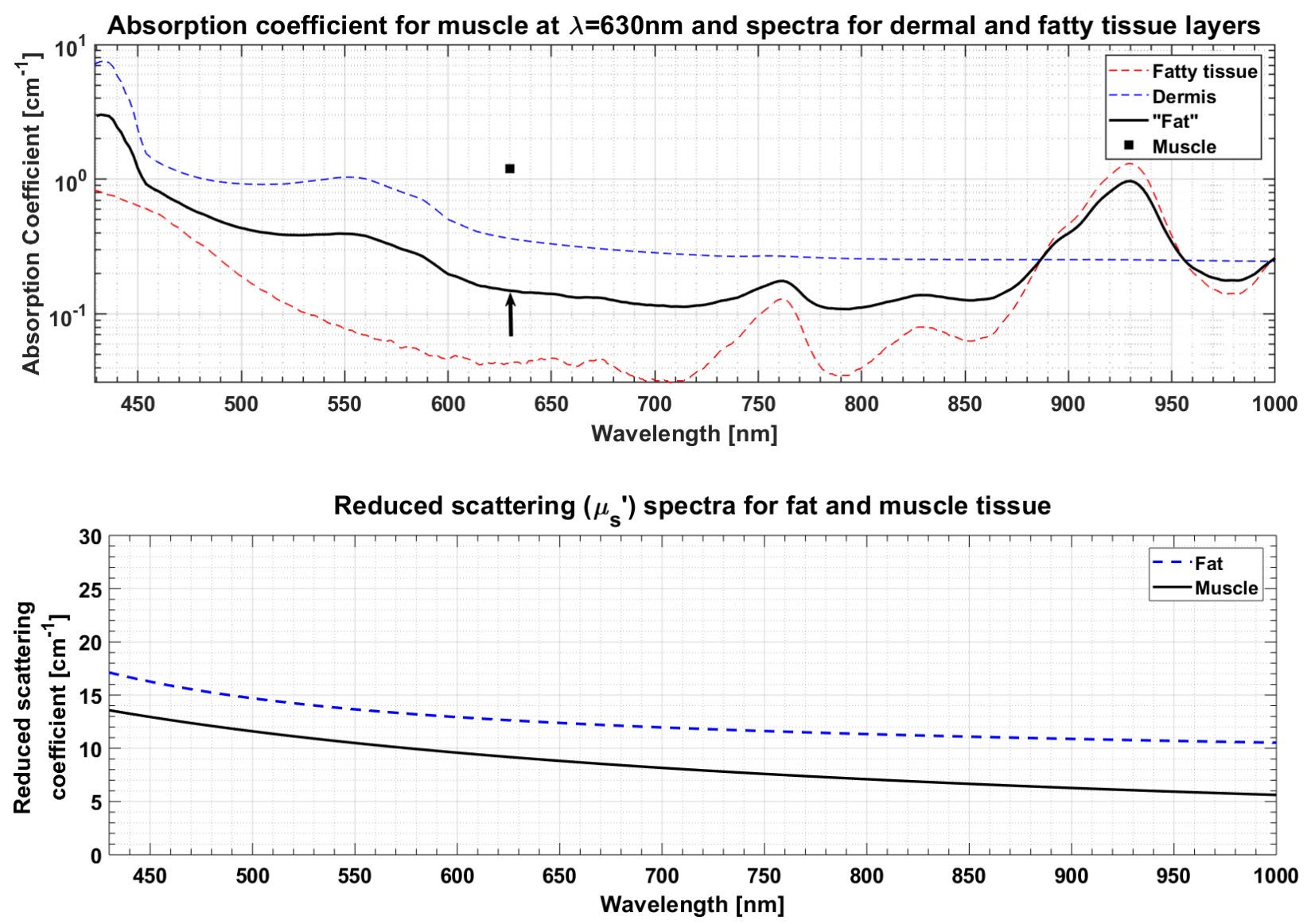

Figure 2.2: TOP: The square data point represents the absorption coefficient of muscle tissue [54]. The absorption spectra of the fatty tissue and dermal layers were plotted using reference values in $[35,52,54,55]$. The black line defining the fat(subcutaneous layer + dermis) is a weighted combination of the dermal $(1 / 3)$ and fatty layers $(2 / 3)$. The arrow marks the target "fat" absorption spectra used for simulation and phantom fabrication. BOTTOM: target reduced-scattering spectra for fat and muscle tissues [52,55]. 


\subsection{Monte Carlo Simulation}

\subsubsection{Simulation Parameters}

The numerical simulation of PA imaging is divided into two parts. The first involves using a Monte Carlo software package known as mcxyz.c software package developed by Steven Jacques, Ting Li and Scott Prahl [63]. The software used Monte Carlo methods to simulate light propagation and energy deposition in a 3D heterogeneous neck tissue model. The second part uses a Matlab code developed by Steven Jacques which uses as input the MC results of the first phase to calculate the photoacoustic signal generated [56]. All runs are carried out by simulating a total of 150 millions $630 \mathrm{~nm}$ photons. The simulations are run serially on an Intel(R)Core(TM)i7-2600 CPU @ 3.40 Ghz. The size of the voxels used in the simulation is $20 \mu m^{3}$. The numbers of voxels in the [x,y] dimensions was [300,300] for all models, and between 800 voxels for the $5 \mathrm{~m} 5 \mathrm{f}$ tissue model, and 1750 for the $10 \mathrm{~m} 20 \mathrm{f}$ model. The simulation times lasted between $\sim 100$ hours for the $5 \mathrm{~m} 5 \mathrm{f}$ model, and $\sim 180$ hours for the $10 \mathrm{~m} 20 \mathrm{f}$ model.

The user-defined $\mu_{a}$ values for the blood(metHb), muscle and fat layers are $100 \mathrm{~cm}^{-1}, 1.2 \mathrm{~cm}^{-1}$ and $0.15 \mathrm{~cm}^{-1}$, respectively. The $\mu_{s}^{\prime}$ values are $10 \mathrm{~cm}^{-1}, 8 \mathrm{~cm}^{-1}$ and $10 \mathrm{~cm}^{-1}$ respectively. An anisotropy factor of $g=0.9$ is used for all tissue types. The top layer of air preceding the skin was given negligible $\mu_{a}$ and $\mu_{s}$ of $10^{-3} \mathrm{~cm}^{-1}$ and $1 \mathrm{~cm}^{-1}$ respectively, and $\mathrm{g}=1.0$

\subsubsection{Data acquisition and analysis}

The mcxyz.c software generates a .bin file representing the light fluence and energy deposition profiles generated within the defined tissue model. The energy deposition profile is then used as input to calculate the PA signal in the tissue. A total of 8 tissue models are used for muscle thickness of $5 \mathrm{~mm}$ and $10 \mathrm{~mm}$ coupled with a top fat layer with thicknesses of 5,10 , 15 and $20 \mathrm{~mm}$. The light fluence, energy absorption and simulated PA signal is generated 
for each for the purpose of establishing trend of peak PA amplitude vs. tissue thickness.

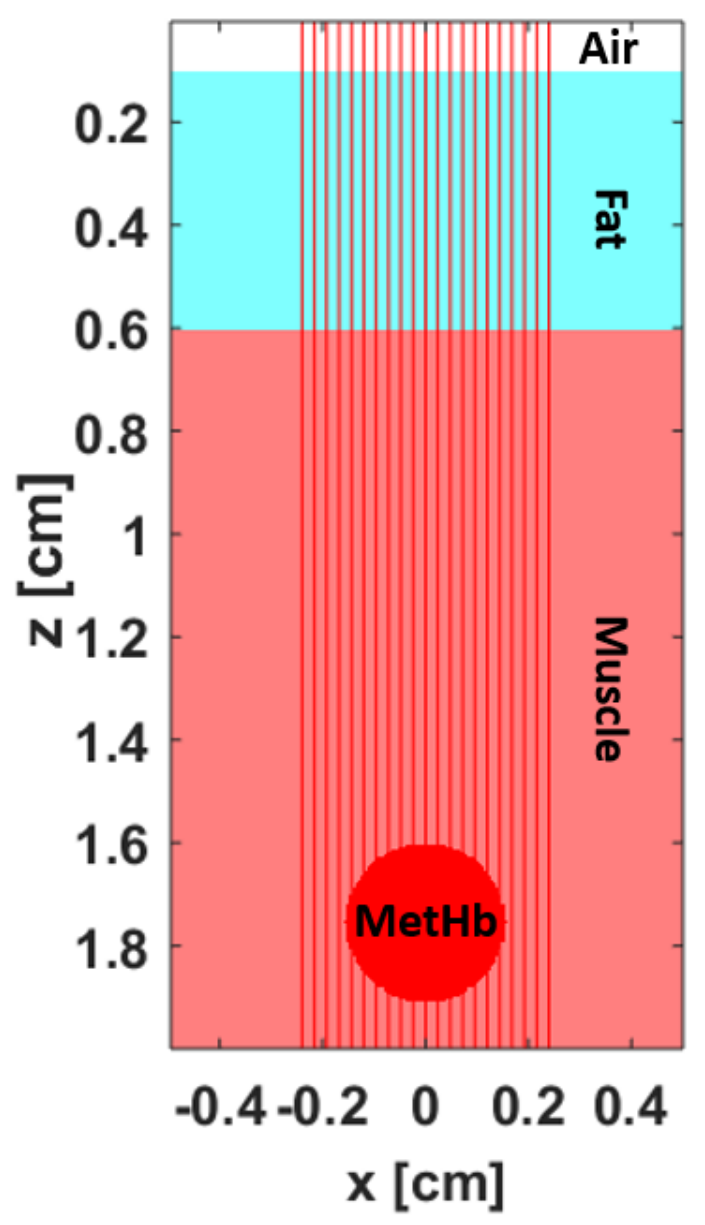

Figure 2.3: A image illustrating a sample neck tissue model used in the MC simulations and experimental PA measurements. The red lines represent unscattered paths of the incident light for illustration purposes. The image was generated using the maketissue.m Matlab file downloaded from [63]

\subsection{Experimental Photoacoustic Measurements}

\subsubsection{Tissue-mimicking phantoms}

Phantoms are commonly used in medical imaging to aid in the the development, testing and characterization of imaging systems or algorithms [64]. A tissue-mimicking phantom emulates important geometrical and/or physical (optical, acoustic etc.) properties of biolog- 
ical tissue for the purpose of providing a relevant imaging environment. PA imaging relies on both the acoustic and optical properties of matter. Compared to optical properties, the acoustic properties of the tissues -for a given frequency- in the skin-to-carotid path are relatively constant [64]. Optical attenuation represents the most significant barrier to adequate SNR detection, and as such, only optical properties are explicitly considered during phantom fabrication.

Hydrogels such as agarose and gelatin are commonly used as PA phantom materials [64]. Gelatin from porcine skin - strength 300 type A is chosen, mainly for its reasonable cost, satisfactory temporal stability, and straightforward fabrication. For background tissue absorption, a stock solution of $1.14 \mathrm{mM}$ Napthol Green B (NGB) dye is used. For tissue scattering, 20\% Intralipid (IL) emulsion is used; IL has negligible absorption ans strong scattering at 630nm [65]. Formaldehyde is used as a stabilizing agent. All the materials listed are from the Sigma-Aldrich brand [66].

\subsubsection{Phantom Fabrication process}

Ultrapure milli-Q water is heated up to $40^{\circ} \mathrm{C}$ in a glass beaker. Gelatin powder is gradually added at a $10 \% \mathrm{~m} / \mathrm{V}(0.1 \mathrm{mg} / \mathrm{ml})$ concentration, with constant stirring. An aluminum foil or saran wrap is used, as shown in Figure 2.4 to cover the top of beaker to minimize evaporation loss. Once the gelatin completely dissolves, the required volume of the $10 \%$ gelatin solution is transferred into a separate beaker, with the top covered at $38^{\circ} \mathrm{C} .1 .14 \mathrm{mM}$ NGB and 20\% IL are slowly added per the concentrations listed for the muscle layer in Table 2.2. Care is taken during this process to minimize air bubble formation. With the beaker covered, the Gelatin+NGB+IL solution is stirred vigorously at $6000-7000 \mathrm{rpm}$ for $25-30$ minutes at $38^{\circ} \mathrm{C}$. The solution is then placed in a $38^{\circ} \mathrm{C}$ water bath, and degassed for 10 minutes to remove trapped air bubbles. Any remaining surface air bubbles are removed using a pipette or a thin napkin. Formaldehyde is then gradually added to the solution, gently stirred for 1 to 2 minutes at $38^{\circ} \mathrm{C}$, and finally poured into a phantom mould - or a small petri dish 
for spectrophotometric measurements (Figure 2.7). The phantom solution is then allowed to cool down and solidify at room temperature for 30 minutes. In the meanwhile, using a clean beaker, the above steps are repeated for the skin/fat layer to be then poured on top of the already solidified muscle layer. If the muscle layer has not yet properly solidified, it is allowed to cool in the fridge for 5 minutes. This is to ensure the liquid skin/fat solution does not mix with any unsolidified muscle tissue. Once the fat layer is poured, the top of the phantom mould is covered with damp tissue, and tightly covered with a saran wrap to conserve humidity and minimize dehydration. The phantom is then allowed to settle at room temperature for 10 minutes before placing it in a refrigerator for 24-48 hours.

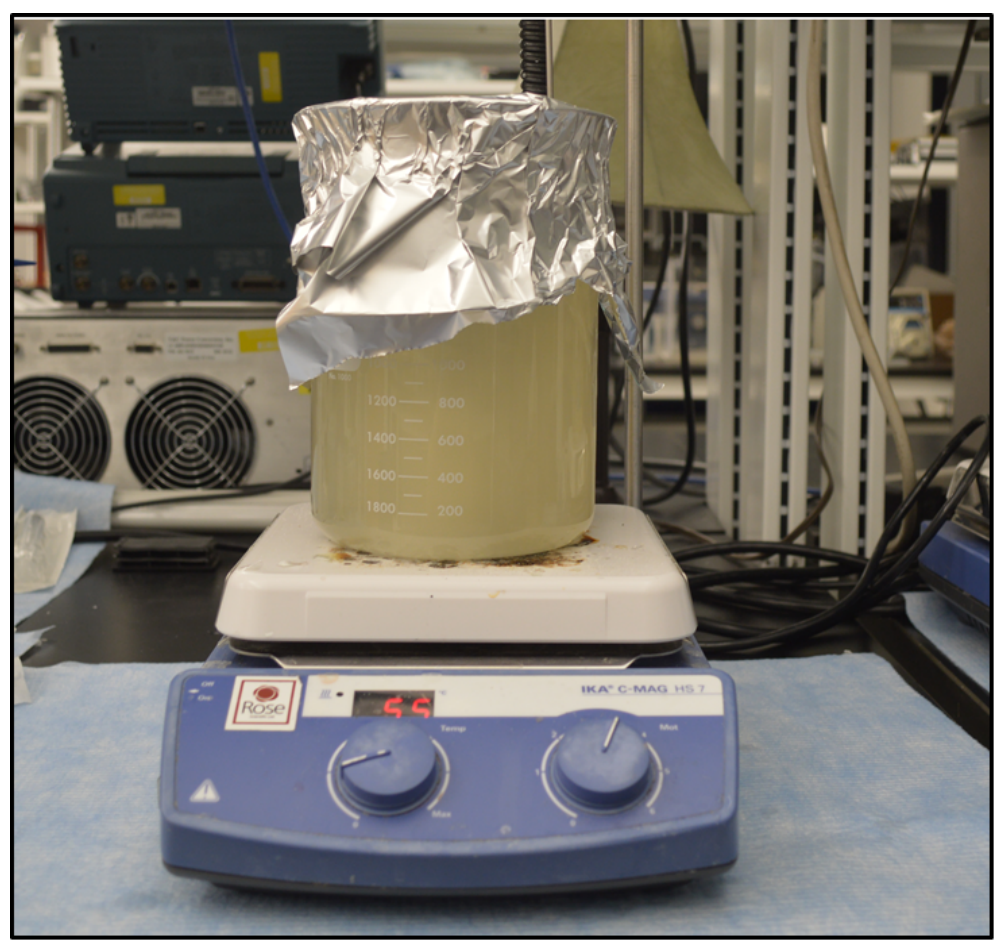

Figure 2.4: Image illustrating the setup for creating the base gelatin solution.

\subsubsection{Validation of optical properties}

Before a phantom is used for PA measurements, spectrophotometric measurements are taken to measure its optical properties. Using a double beam integrating sphere (Shimadzu 3600 


\begin{tabular}{|l|c|c|}
\hline & Fat & Muscle \\
\hline 1.14 mM NGB & $0.35 \%$ & $8.0 \%$ \\
\hline 20\% Intralipid & $10.0 \%$ & $8.0 \%$ \\
\hline Fomraldehyde & $2.0 \%$ & $2.0 \%$ \\
\hline
\end{tabular}

Table 2.2: Volume fractions defined as volume of ingredient $V_{i}$ divided by the total volume of solution $V_{T}: \frac{V_{i}}{V_{T}} \%$ of the ingredients used to create the skin/fat and muscle layers in the tissue-mimicking phantoms.
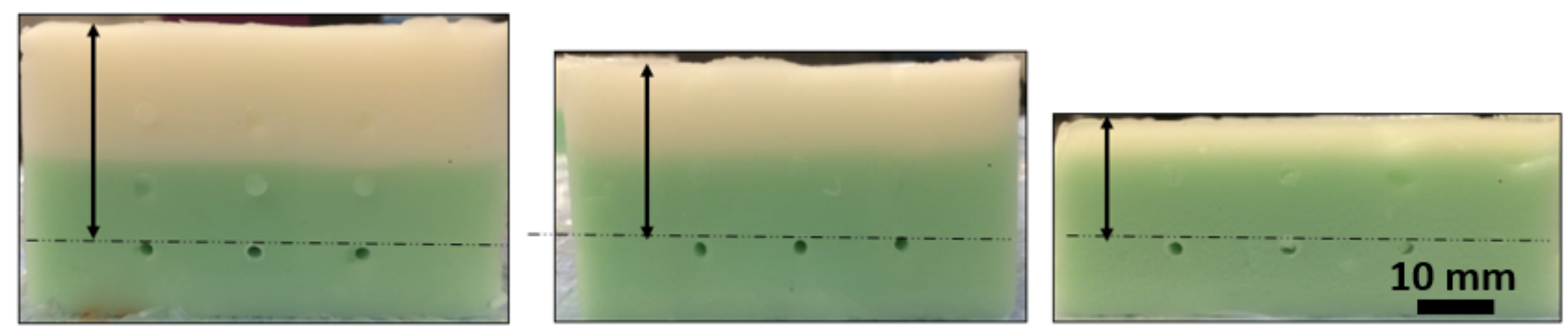

Figure 2.5: Left to Right: completed tissue-mimicking phantoms with $3.1 \mathrm{~mm}-$ diamter vessels at 25, 20 and $15 \mathrm{~mm}$ depths. All have 10mm thick musclemimicking tissue (green) with 15,10 , and $5 \mathrm{~mm}$ thick skin/fat-mimicking tissue (white) on top. The middle vessel is typically used to inject the metHb; the other two are for redundancy.

UV-VIS-NIR Spectrophotometer), diffuse reflectance (DR) and total transmittance (TT) spectra measurements are taken of small phantom samples in the 400-800 $\mathrm{nm}$ range. The phantom samples, as shown in Figure 2.5 were circularly-shaped, able to adhere to the walls of integrating sphere while covering the entirety of the sample port seen in the schematic Figure 2.6. This removed the need for using glass microscope slides to sandwich the samples for measurements. Using the Beer-Lambert law and Inverse Adding Double (IAD) technique, the measured DR and/or TT values were used to calculate absorption and reduced scattering coefficients [67-69].

\begin{tabular}{|c|c|c|c|c|c|}
\hline \multicolumn{2}{|c|}{} & \multicolumn{4}{|c|}{ Fat $\mathbf{( m m})$} \\
\cline { 3 - 6 } \multicolumn{2}{|c|}{} & $\mathbf{5}$ & $\mathbf{1 0}$ & $\mathbf{1 5}$ & $\mathbf{2 0}$ \\
\hline \multirow{2}{*}{ Muscle } & $\mathbf{5}$ & $5 m 5 f^{1,2}$ & $5 m 10 f^{1,2}$ & $5 m 15 f^{1,2}$ & $5 m 20 f^{1,2}$ \\
\cline { 2 - 6 } & $\mathbf{1 0}$ & $10 m 5 f^{1,2}$ & 10m10f $^{1,2}$ & $10 m 15 f^{1}$ & $1^{10 m 20 f^{1}}$ \\
\hline
\end{tabular}

Table 2.3: A list of the phantom/tissue models created for ${ }^{1}$ Monte Carlo and ${ }^{2}$ experimental studies. 
The extinction coefficient $\mu_{t}=\mu_{a}+\mu_{s}$ simplifies to $\mu_{a}$ in a low-scattering regimez $\mu_{s} \sim 0$, allowing the use Beer-Lambert law to calculate $\mu_{a}$ from the TT measurements [71,72]. The phantom base, made from $10 \%$ gelatin, has negligible scattering. Given the negligible absorption of intralipid and formaldehyde at $630 \mathrm{~nm}$ [73], the optical absorption of the phantoms was hence approximated to that of the NGB dye in gelatin, i.e $\mu_{t} \approx \mu_{a}$ where $\mu_{a: \text { Tissue }} \approx \mu_{a: N G B}$ [71]. The TT spectra of gelatin mixed with NGB in a 1.0-cm path quartz cuvette is mea-

sured, and the optical absorption coefficient is then calculated by: $\mu_{a}=\frac{\ln (T T \%)}{-d}$, where $d=1.0 \mathrm{~cm}$ defines the thickness of the sample. The IAD method developed by Scott Prahl was then used to take DR and TT spectra and iteratively calculate the scattering spectra for the phantom samples [68]. More details on the method and the user-defined settings used for the IAD software can be found in Appendix B.
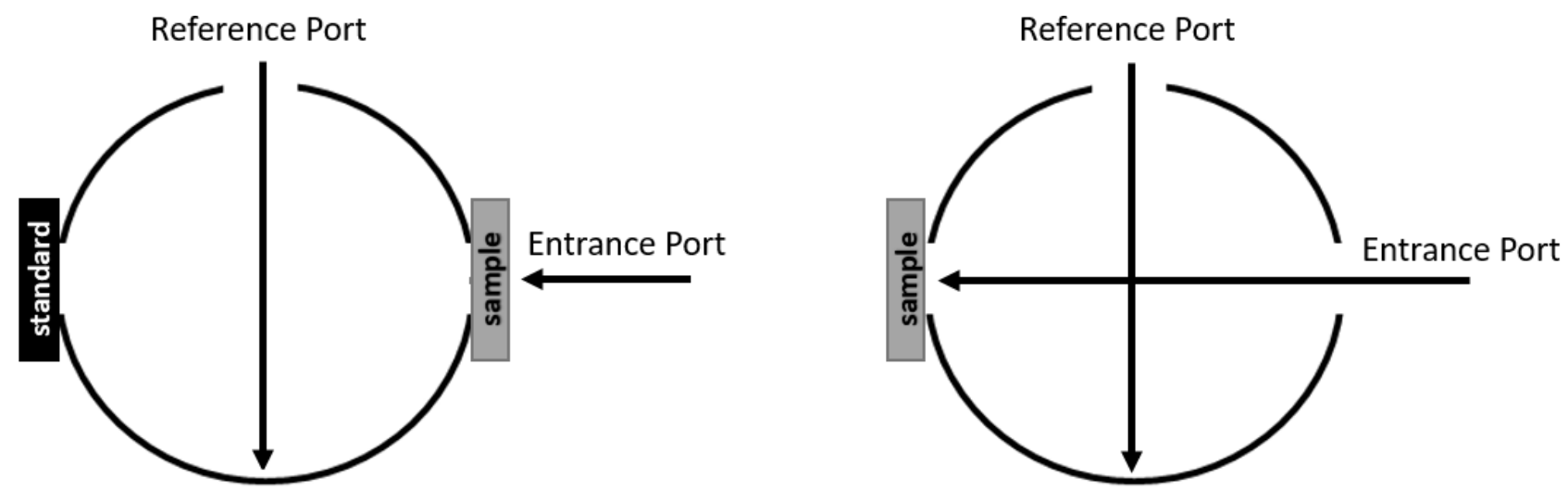

Figure 2.6: The integrating sphere setup used for the Diffuse Reflectance (left) and Total Transmittance (right) measurements.

\subsubsection{Methaemoglobin as absorbing target}

Methemoglobin (MetHb) is the primary source of contrast in MR imaging of IPH, and is the proposed target of contrast for PA imaging of IPH. The observed of concentrations of in-vivo metHb in IPH are between 9mmol. $L^{-1}$ and 15 mmol. $L^{-1}$ [27]. Assuming that the linearity between absorbance and concentration is maintained at these concentrations, we 


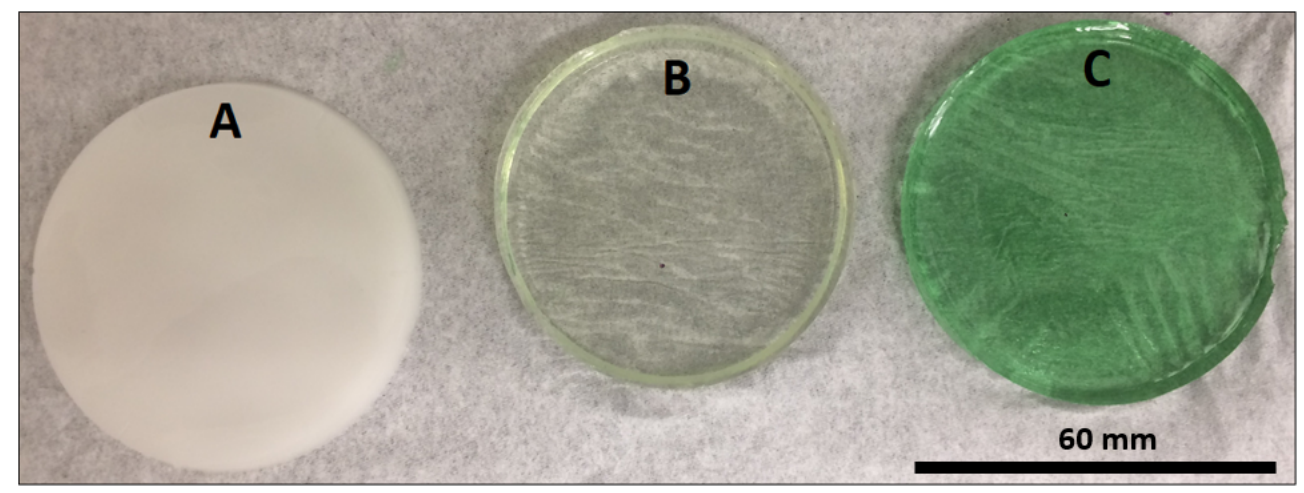

Figure 2.7: Phantom samples used for spectrophotometric measurements. A:Fat mimicking, B: Fat absorption (only) mimicking. C: Muscle absorption (only) mimicking.

estimate the resulting absorption coefficients by $\mu_{\mathbf{a}}=\ln (\mathbf{1 0}) \cdot \mathbf{C} \cdot \varepsilon$ where $C$ is the concentration of the absorber and $\varepsilon$ is the molar extinction -or absorption- coefficient. Given the reported range of $C$ between $9 m M$ and $15 \mathrm{mM}$, and the molar extinction coefficient of at $15000 \mathrm{~cm}^{-1} \mathrm{mmol}^{-1} \mathrm{~L}$ at $630 \mathrm{~nm}$ (Figure $\mathbf{1 . 7}$ ), the resulting absorption coefficients are between $310 \mathrm{~cm}^{-1}$ and $518 \mathrm{~cm}^{-1}$.

Human blood provided by the Canadian Blood Services was used as the source of blood. The blood initially consists predominantly of oxyHaemoglobin (OxyHb), which was then converted to MetHb. This was done via a two-step process. First, the blood was centrifuged at $1300 \mathrm{~g}$ for 10 minutes to fractionate the blood into its three main constitutes; red blood cells (RBCs), plasma and a buffy coat, as shown in Figure 2.8. The RBCs layer was carefully separated and gently mixed with Sodium Nitrite $\left(\mathrm{NaNO}_{2}\right)$ to convert the present oxyHb to MetHb [74]. The Nitrite anion $\mathrm{NO}_{2}^{-}$acts as an oxidizing agent as it converts the iron in the heme group from its ferrous $\left(\mathrm{Fe}^{2+}\right)$ form to its ferric $\left(\mathrm{Fe}^{3+}\right)$ form, forming Nitrogen dioxide $\left(\mathrm{NO}_{2}\right)$ as a result [74]. To confirm the presence and absorption strength of MetHb, the TT spectra were measured and used to calculate the absorption spectra. A lowscattering regime needed for the Beer-Lambert approximation is ensured by using optically thin $(100-\mu m)$ thick quartz cuvette. 


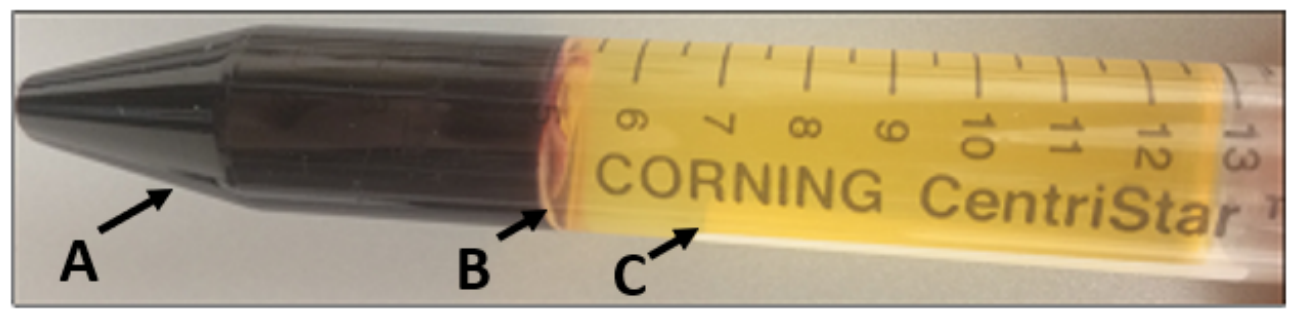

Figure 2.8: Fractionated whole blood. The supernatant Plasma layer (C) is separated from the precipitate RBCs layer (A) via a buffy coat (B).

\subsubsection{Experimental PA setup}

There are three components to the experimental setup; laser generation, phantom placement, and Photoacoustic measurement. An OPOTEK INC. Radiant series tunable laser system is used to provide a laser beam with a spot size with a diameter of $4.78 \mathrm{~mm}$ at the phantom surface. The laser pulse widths were between $4 \mathrm{~nm}$ and $6 \mathrm{~nm}$ fired at a repetition rate of $10 \mathrm{~Hz}$. The power output ranged between $20 \mathrm{~mW}$ and $25 \mathrm{~mW}$ at $630 \mathrm{~nm}$, giving an irradiance $E_{e}$ ranging from $111 \mathrm{~mW} / \mathrm{cm}^{2}$ to $138 \mathrm{~mW} / \mathrm{cm}^{2}$. The laser system was synced to Vantage 128 Verasonics system used to record and display the measured PA signals acquired via a 128-element linear L7-4 transducer, with a field-of-view of 38mm and central frequency of $\sim 5$ Mhz. Figure 2.9 shows the experimental setup used; the phantom was positioned on its side laterally with respect to the vessels, with the fat layer oriented to face the laser beam, whereas the transducer is placed laterally behind the phantom along the length of the vessel. The transducer is not fixed as shown in Figure 2.9, but is rather guided with the hand for free hand guided measurements. The height at which the phantoms are positioned was adjusted such that center of the laser spot strikes the vertical mid-point of thes center vessel. The phantom itself was moved horizontally across the length of the vessel between different measurement trials such that laser irradiated 3 points equidistant form each other along the length of the vessel, labeled by the $\mathbf{B}_{\mathbf{1}}, \mathbf{B}_{\mathbf{2}}$ and $\mathbf{B}_{\mathbf{3}}$ in $\mathbf{F i g u r e} \mathbf{2 . 1 0}$.

It is noted that the proposed setup is not a clinically viable one. In a clinical setting, the transducer would be on the skin surface, i.e. on the laser side. 


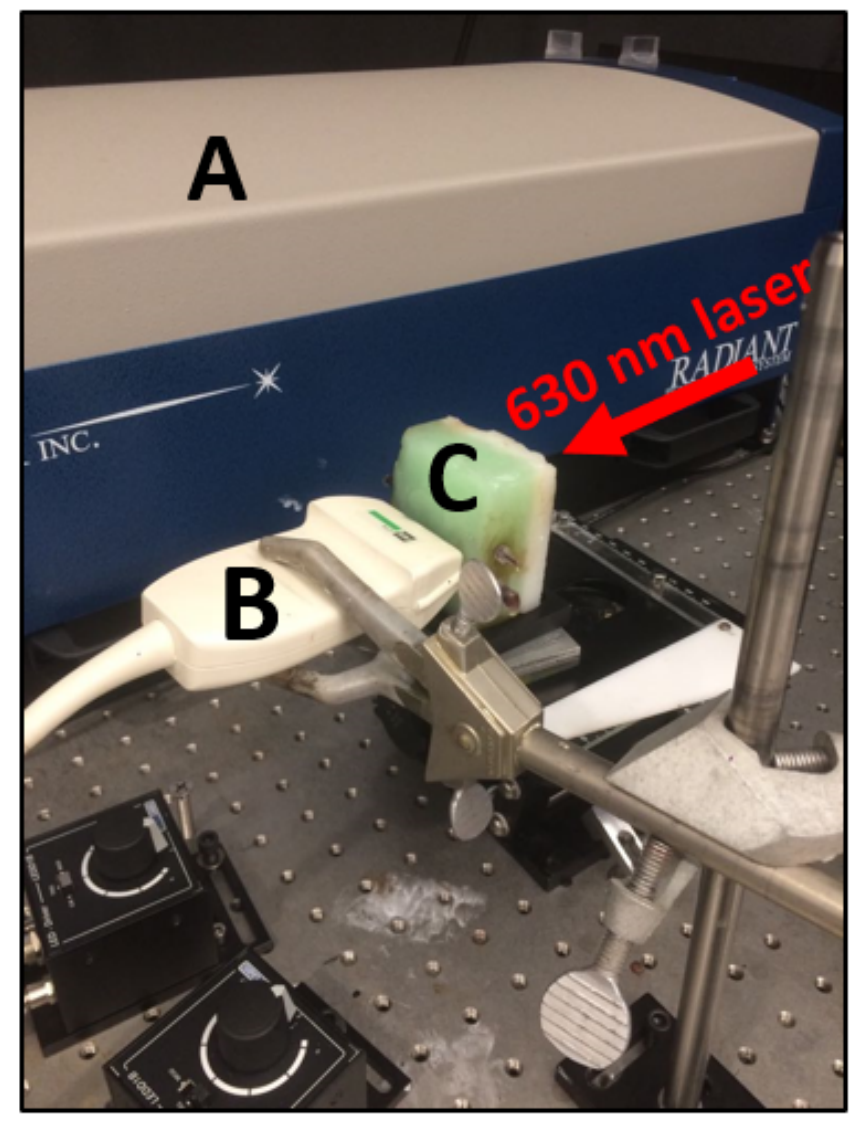

Figure 2.9: The experimental setup for PA measurements of the tissue-mimicking phantoms. A - Opotek INC. Radiant tunable laser system. B- L7-4 transducer connected to a Vantage 128 Verasonics system. C- Tissue-mimicking phantom. The red arrows illustrate the direction of the laser beam.

\subsubsection{Measurement and Analysis}

\subsubsection{Data Acquisition}

Once a MetHb sample is injected into the center vessel, and phantom placed in position, the laser is turned on and allowed to reach its maximum energy level. The Verasonics Photoacoustics Matlab script is then initiated, and with the transducer in hand, the live 2D image display generated by the Matlab script is used to guide the free-hand PA measurements to probe for regions of high SNR. $\mathbf{N}=\mathbf{1 0}$ measurements (or trial) are taken per phantom, wherein each trial constitutes of $\mathbf{F}=\mathbf{2 0 0}$ frames for the $\mathbf{E}=\mathbf{1 2 8}$ transducer elements, i.e. each phantom measurement records a total of $\mathbf{N} \times \mathbf{F} \times \mathbf{E}=\mathbf{1 0} \times \mathbf{2 0 0} \times \mathbf{1 2 8}=\mathbf{2 5 6 0 0 0 \mathrm { RF }}$ 


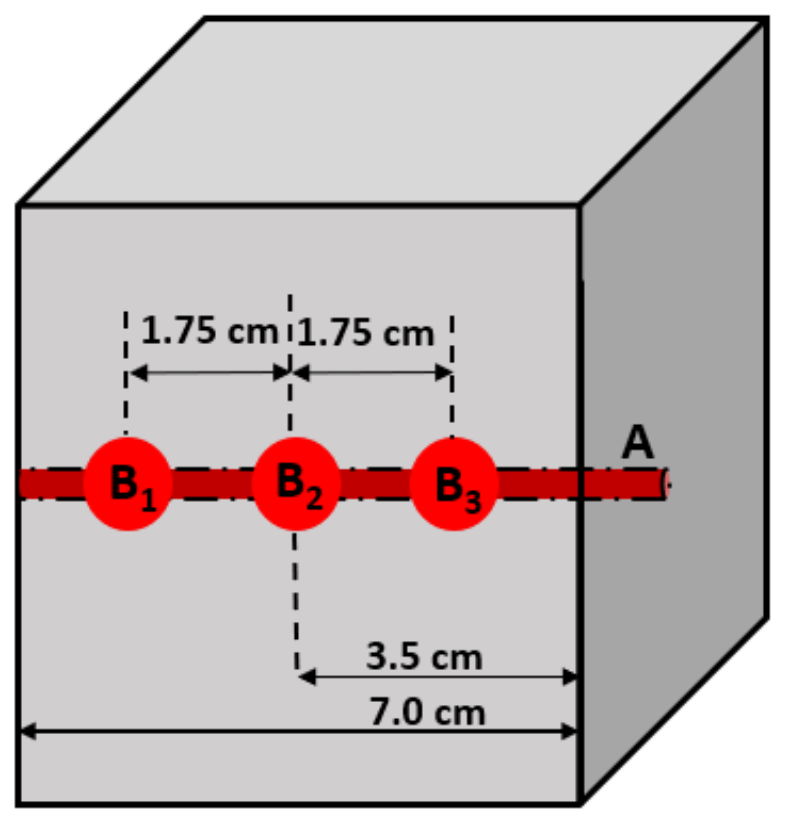

Figure 2.10: An model illustration of the position of the phantom with respect to the laser spot. The phantom is positioned such that the laser spot strikes the vertical mid-point of the vessel, marked by $\mathbf{A}$. Ten measurement trials are completed per phantom for a given MetHb sample, where 3 trials are taken at positions $\mathbf{B}_{\mathbf{1}}$ and $\mathbf{B}_{\mathbf{3}}$, and 4 trials are taken at mid-position marked $\mathbf{B}_{\mathbf{2}}$.

lines. Referring to Figure $\mathbf{2 . 1 0}, 3$ trials are taken at each of the positions $\mathbf{B}_{\mathbf{1}}$ and $\mathbf{B}_{\mathbf{3}}$, and 4 trials are taken at mid-position marked $\mathbf{B}_{\mathbf{2}}$. Figure $\mathbf{2 . 1 1}$ shows an example of two frames from the live $2 \mathrm{D}$ display. The purpose of the free-hand approach is to mimic the approach used in the clinical setting, where a live-feed display is used to help the user guide the transducer by hand towards the high SNR regions of interest.

\subsubsection{Data Analysis}

Each measurement event or trial has 256000 RF lines. A sample RF line is shown in Figure 2.12. Given the motivation to capture high SNR events, the signal RF lines are sorted in order of the peak amplitude corresponding to the signal from the MetHb in the vessel, labeled by $D$ in Figure 2.12. A background noise region is defined within region labeled by $B$. The SNR in decibels $(\mathrm{dB})$ is then calculated as the ratio of 20 times the common 

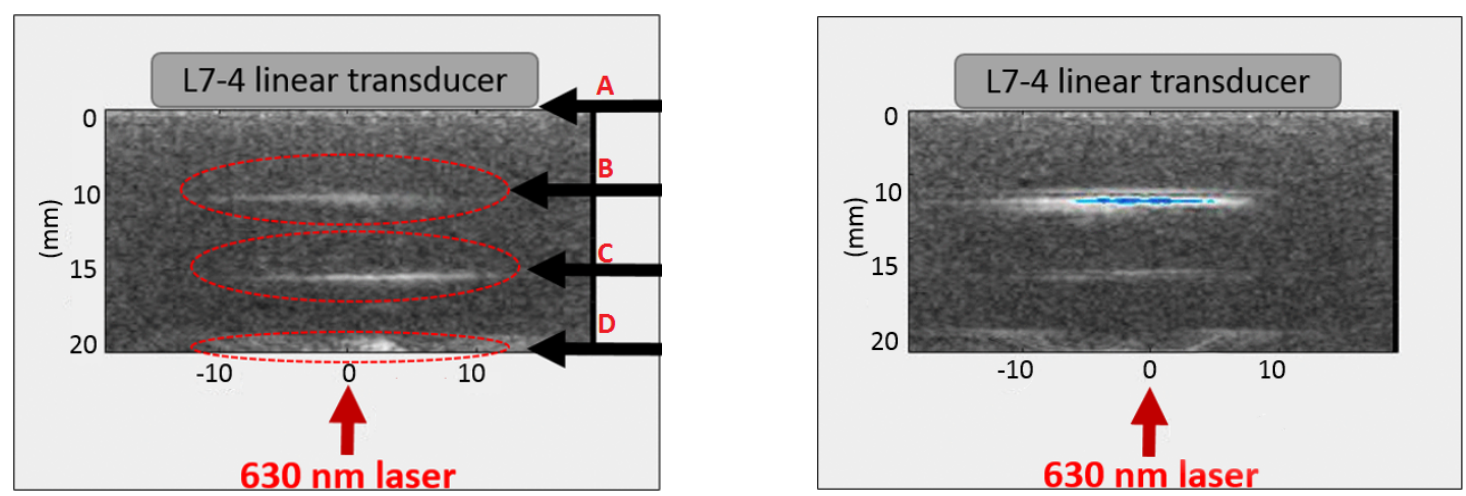

Figure 2.11: A snapshot showing two individual frames (out of a total of 200 per trial) of the live $2 \mathrm{D}$ image view used to guide the free-hand measurement of the PA signals. A- Bottom surface of phantom, i.e. transducer side shown in Figure 2.9. B- The PA signal from the upper boundary of the vessel, i.e. the boundary closer to the laser. C- The PA signal from the fat/muscle boundary. D- Signal from the top surface of the phantom, i.e. the laser side.

logarithm of the ratio of $D$ to the standard deviation of $B$ of the analytic signal where $\operatorname{SNR}(\mathbf{d B})=\mathbf{2 0} \log \left(\frac{\mathbf{D}}{\sigma_{\mathrm{B}}}\right)$. A value $\mathbf{n}$ is used to define the top SNR measurements out of the 256000 SNRs. The value $\mathbf{n}$ is defined as the maximum number of measurements needed to maintain a coefficient of variation $\mathrm{CV} \leq 10 \%$. The calculated SNRs are then used to plot SNR against MetHb absorption, as well as SNR against Fat Thickness. The former allows for estimating SNR at desired MetHb absorptions ranging between $310 \mathrm{~cm}^{-1}$ and $518 \mathrm{~cm}^{-1}$. The latter allows for estimating the maximum fat thickness (and imaging depth) for a minimum detectable SNR of $\sim 12 d B$. 


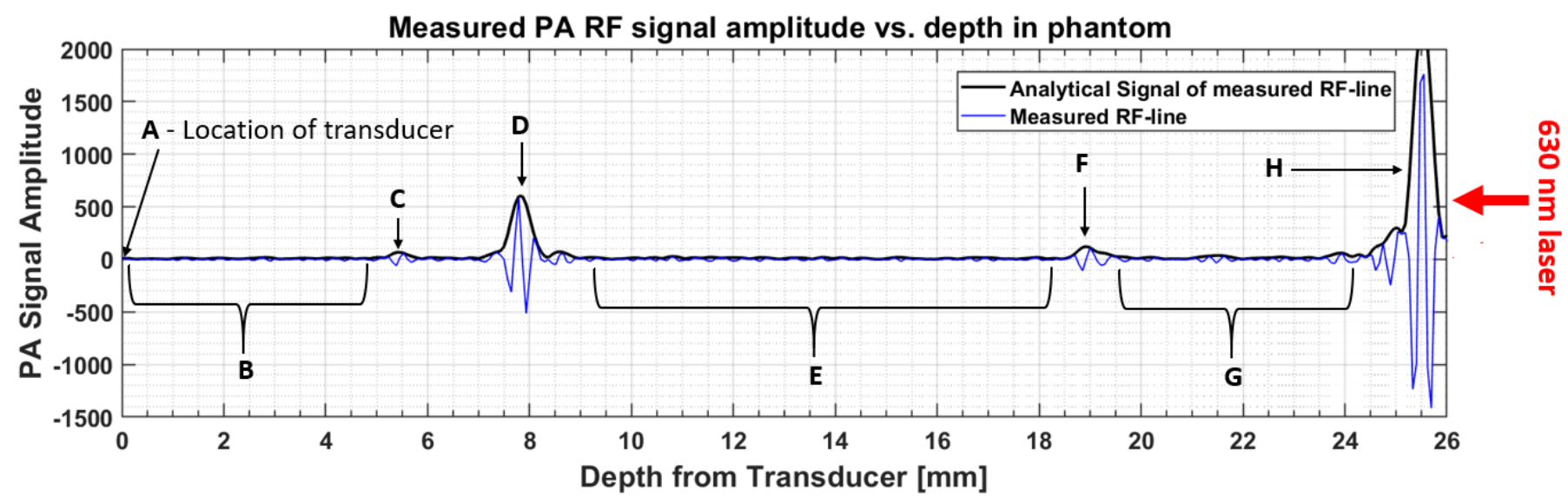

Figure 2.12: A labeled example of an experimentally measured PA RF line. (A)- Location of transducer, (B)- Background noise, (C)- Signal from the lower vessel boundary, (D)Signal from the upper vessel boundary, (E)- Muscle region, (F)- Signal from the fat/muscle boundary, (G)- Fat region and (H)- Signal from the phantom surface. The SNR per measurement is taken as 20 times the natural logarithm of ratio of the peak signal at the upper boundary of vessel to the standard deviation of background noise: $\mathbf{S N R}=\mathbf{2 0} \log \left(\frac{\mathbf{D}}{\sigma_{\mathrm{B}}}\right)$ 


\section{Chapter 3}

\section{Results}

\subsection{Properties of Phantoms and MetHb}

\subsubsection{Absorption spectra of Whole Blood and MetHb}

The absorption spectra for human whole blood pre-and post-centrifugation, and after its final conversion to methaemoglobin are displayed in Figure 3.1. The haemoglobin in the whole blood is initially mostly $\mathrm{OxyHb}$, as inferred by the characteristic double peaks between 540nm and 580nm. After centrifugation, cellular deformation might have occurred, leading to the flattening of the oxyhaemoglobin peaks, with some possible associated MetHb formation, albeit in small concentrations. This was inferred from the formation of a peak at $\sim 630 \mathrm{~nm}$. Finally, after the conversion to MetHb, the absorption coefficient at $630 \mathrm{~nm}$ increases over twofold.

Making MetHb blood samples with reproducible absorptions was challenging, due to factors such as the age of the blood. The absorption coefficients $\mu_{a}$ were 12, 30, 90, 132 and $175 \mathrm{~cm}^{-1}$. Producing MetHb samples of stronger absorption was not possible; attempts at promoting further MetHb conversion would ultimately lead to excessive clotting and coagulation of the blood into a semi-solid state, making it challenging to inject. 


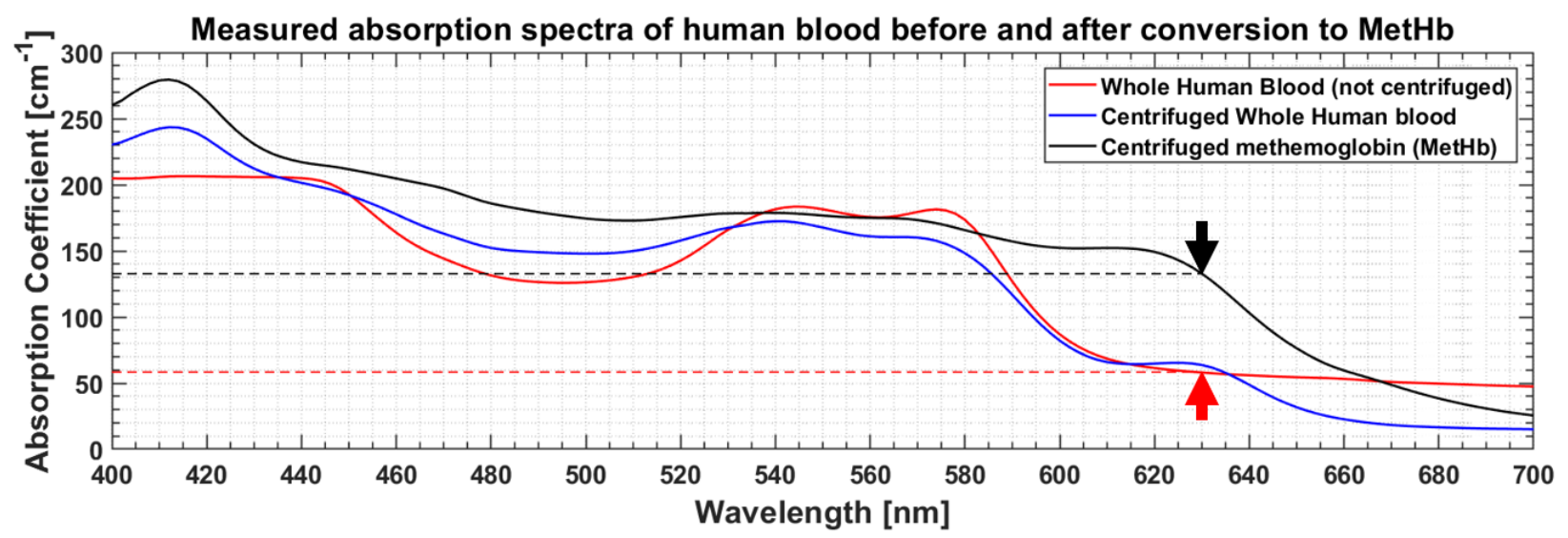

Figure 3.1: The measured absorption spectra of human whole blood. Initially, the whole blood (RED) is predominantly oxygenated, as noted by the characteristic OxyHb double peak between $540 \mathrm{~nm}$ and $580 \mathrm{~nm}$. The presence of MetHb (BLACK) can be inferred by the characteristic peak at $\sim 630 \mathrm{~nm}$. The absorption of MetHb at $630 \mathrm{~nm}$ (black dotted line) is approximately 2.3 times greater than that of the oxygenated whole blood (red dotted line).

\subsubsection{Measured spectra of tissue-mimicking phantoms}

The measured TT and DR Spectra (Appendix D) of the tissue mimicking phantoms were used via the IAD method to calculate the the absorption and reduced scattering spectra shown in

Figure 3.2. The calculated absorption and reduced scattering coefficients for the fat layer at $630 \mathrm{~nm}$ were $\mu_{a}=0.168 \pm 0.0438 \mathrm{~cm}^{-1}$ and $\mu_{s}^{\prime}=11.0 \pm 2.95 \mathrm{~cm}^{-1}$, whereas the measured absorption and reduced scattering coefficients for the muscle layer are $\mu_{a}=1.19 \pm 0.163$ $\mathrm{cm}^{-1}$ and $\mu_{s}^{\prime}=9.51 \pm 4.26 \mathrm{~cm}^{-1}$. For context, the target absorption and reduced scattering coefficients for fat at $630 \mathrm{~nm}$ are $\mu_{a} \approx 0.15 \mathrm{~cm}^{-1}$ and $\mu_{s}^{\prime} \approx 10.0 \mathrm{~cm}^{-1}$, and for the muscle $\mu_{a}=1.2 \mathrm{~cm}^{-1}$ and $\mu_{s}^{\prime}=8.0 \mathrm{~cm}^{-1}$. Table 3.1 lists the measured optical properties against the target values, and the $\%$ difference between the two.

\subsubsection{US Images of tissue-mimicking phantoms}

Before PA measurements and injection of MetHb, the dimensions (thicknesses) of the phantoms were verified. This was done via B-mode (2D) ultrasound imaging; Figure 3.3 shows the photo of a fabricated 10m10f phantom and its corresponding US B-mode image. A total 

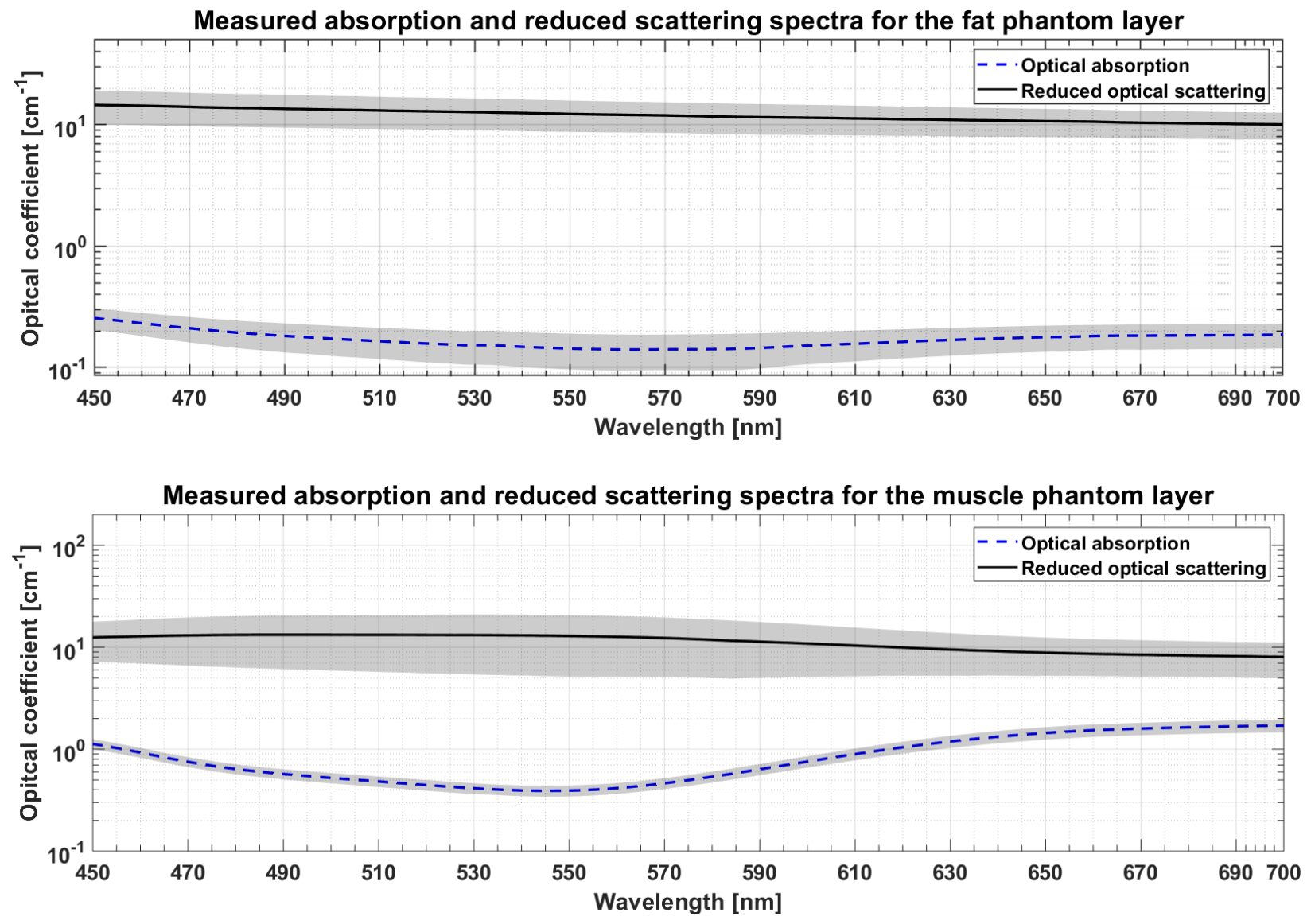

Figure 3.2: TOP: Measured average absorption and reduced scattering spectra of the fat phantom layer. The shaded area represents the standard deviation for 14 scattering measurements, and 10 absorption measurements of different phantom samples. Bottom: Measured average absorption and reduced scattering spectra of the muscle phantom layer. The shaded area represents the standard deviation for 5 scattering measurements and 10 absorption measurement of different phantom samples. 


\begin{tabular}{|l|c|c|c|}
\hline & Target $\left(\mathbf{c m}^{-1}\right)$ & Measured $\left(\mathbf{c m}^{-1}\right)$ & $\mid$ Percentage Difference $\% \mid$ \\
\hline Muscle $\mu_{a}$ & 1.2 & 1.19 & 0.833 \\
\hline Muscle $\mu_{s}^{\prime}$ & 8.0 & 9.51 & 18.9 \\
\hline Fat $\mu_{a}$ & 0.15 & 0.168 & 12.0 \\
\hline Fat $\mu_{s}^{\prime}$ & 10.0 & 11.0 & 10.0 \\
\hline
\end{tabular}

Table 3.1: A summary of the measured average optical properties compared to the target optical properties of fat and muscle tissue layers. The absolute $\%$ difference off the reference values range between $0.83 \%$ for muscle absorption and $18.9 \%$ for muscle reduced scattering.

of 21 phantoms defining 6 different tissue combinations (Table 2.3) were produced for the experimental PA measurements.
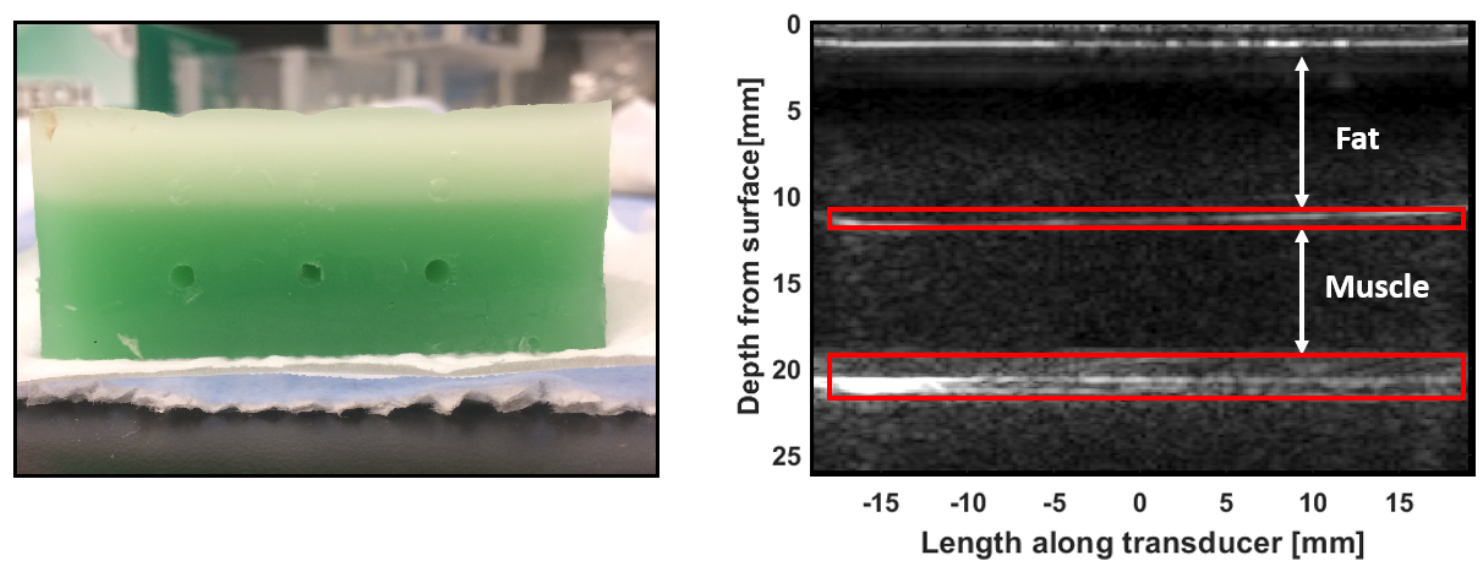

Figure 3.3: A photographed imaged of a fabricated 10m10f phantom, and the corresponding US B-Mode image on the right. The highlighted regions demonstrate the fat-to-muscle transition on the top, and the $\sim 3.1 \mathrm{~mm}$ wide vessel on the bottom. 


\subsection{Monte Carlo Simulation Results}

The mcxyx Monte Carlo software produces 2D profiles of light fluence and energy distribution within a tissue model. An example is shown in Figure 3.4. On the top right side of the same figure are line plots of light fluence and energy distribution vs. depth along the central axis $(\mathrm{x}=0 \mathrm{~cm})$. The bottom of Figure 3.4 plots the simulated pressure signal amplitude vs. depth normalized to the maximum amplitude at the vessel boundary. Note that the $2 \mathrm{D}$ profiles start from $\mathrm{z}=0$ with the skin surface being at $0.1 \mathrm{~cm}$, or $1 \mathrm{~mm}$. The corresponding line plots, however, start from $-0.1 \mathrm{~cm}$ or $-1 \mathrm{~mm}$, with the skin or "fat" surface defining the zero point. The relevant regions of interest, marked by arrows on the plots, are the boundary points where transitions in absorption coefficients occur, i.e. the fat-to-muscle boundary, the vessel's upper boundary (muscle-to-MetHb), and the vessel's lower boundary. These regions demonstrate local changes in energy deposition, and consequently, pressure generation.

A visual side-by-side comparison between the 2D fluence and energy deposition profiles for the $5 \mathrm{~m} 5 \mathrm{f}$ and $5 \mathrm{~m} 10 \mathrm{f}$ phantoms is shown in Figure 3.5. Distribution profiles for other tissue models, including 10mm-muscle phantoms, can be found in Appendix C. A comparison between the fluence, energy and PA amplitude vs. depth for the 5m5f, 5m10f, 5m15f and 5m20f simulations is shown in Figure 3.6. All plots are taken at the central axis $(\mathrm{x}=0 \mathrm{~cm})$. The fluence and energy lines follow a linear decay on the log-scale due the exponential attenuation of light in tissue. In all simulations, a small increase in energy deposition is observed at boundaries between fat and muscle. A larger increase is observed between the muscle and vessel boundary (marked by solid arrows in Figure 3.5). It is at this muscle/vessel boundary that the target PA pressure signal is generated. In the 2D energy deposition profiles, this boundary is marked by a red ring-like boundary around the vessel. Past the boundary, fluence and energy deposition progressively decrease to a minimum at the mid-vessel point, where the fluence and energy deposition are $\sim 8$-10 orders of magnitude lower relative to the maximum at the vessel's top boundary. Past the mid-vessel point, the fluence and energy 
deposition increase up until the vessel's lower boundary. At the lower vessel boundary, the signals $\sim 2$ orders of magnitude lower relative to the upper boundary.

The bottom plot in Figure 3.6 shows a semi-log plot of PA pressure signal amplitudes generated at the vessel in different tissue models, normalized to the maximum in the $5 \mathrm{~m} 5 \mathrm{f}$ model. The solid arrows mark the upper vessel boundaries, while the dotted arrow lines mark the lower vessel boundaries. With increasing tissue thickness, the PA amplitudes decrease exponentially, as implied (linearly in the log-scale). Table 3.2 lists the PA peak amplitudes for the different tissue models normalized to the maximum in $5 \mathrm{~m} 5 \mathrm{f}$ in Figure 3.7. The normalized PA peak amplitudes are plotted against fat thickness for 5mm-muscle and 10mm-muscle phantoms. The values for attenuation (per mm-fat) in the 5mm-muscle and the 10mm-muscle phantoms is defined by the slopes of the linear beset-fit lines in Figure 3.7: $1.63 d B . \mathrm{mm}^{-1}$ and $1.75 d B . \mathrm{mm}^{-1}$ respectively.

Higher degrees of numerical error and fluctuations are observed for fluence and energy deposition within the vessel. This is possibly due to the lower fluence within the vessel, as well as the much lower free mean path $1 / \mu_{t}$ of the photons due to the higher $\mu_{t}^{\prime}=\mu_{a}+\mu_{s}^{\prime}=110 \mathrm{~cm}^{-1}$ relative to $\mu_{t}^{\prime}=10.15 \mathrm{~cm}^{-1}$ for fat, and $\mu_{t}^{\prime}=9.2 \mathrm{~cm}^{-1}$ for muscle. A greater $\mu_{t}$ would require smaller simulation steps (voxel sizes) [70].

\begin{tabular}{|l|c|c|c|c|c|}
\hline \multicolumn{2}{|c|}{} & \multicolumn{4}{|c|}{ Fat $\mathbf{( m m})$} \\
\cline { 3 - 6 } \multicolumn{2}{|c|}{} & $\mathbf{5}$ & $\mathbf{1 0}$ & $\mathbf{1 5}$ & $\mathbf{2 0}$ \\
\hline \multirow{2}{*}{ Muscle (mm) } & $\mathbf{5}$ & 0 & -7.34 & -15.34 & -24.29 \\
\cline { 2 - 6 } & $\mathbf{1 0}$ & -19.79 & -28.96 & -37.71 & -46.12 \\
\hline
\end{tabular}

Table 3.2: Monte Carlo simulated PA amplitudes (dB) in 8 tissue models normalized to the maximum in the $5 \mathrm{~m} 5 \mathrm{f}$ simulation. 

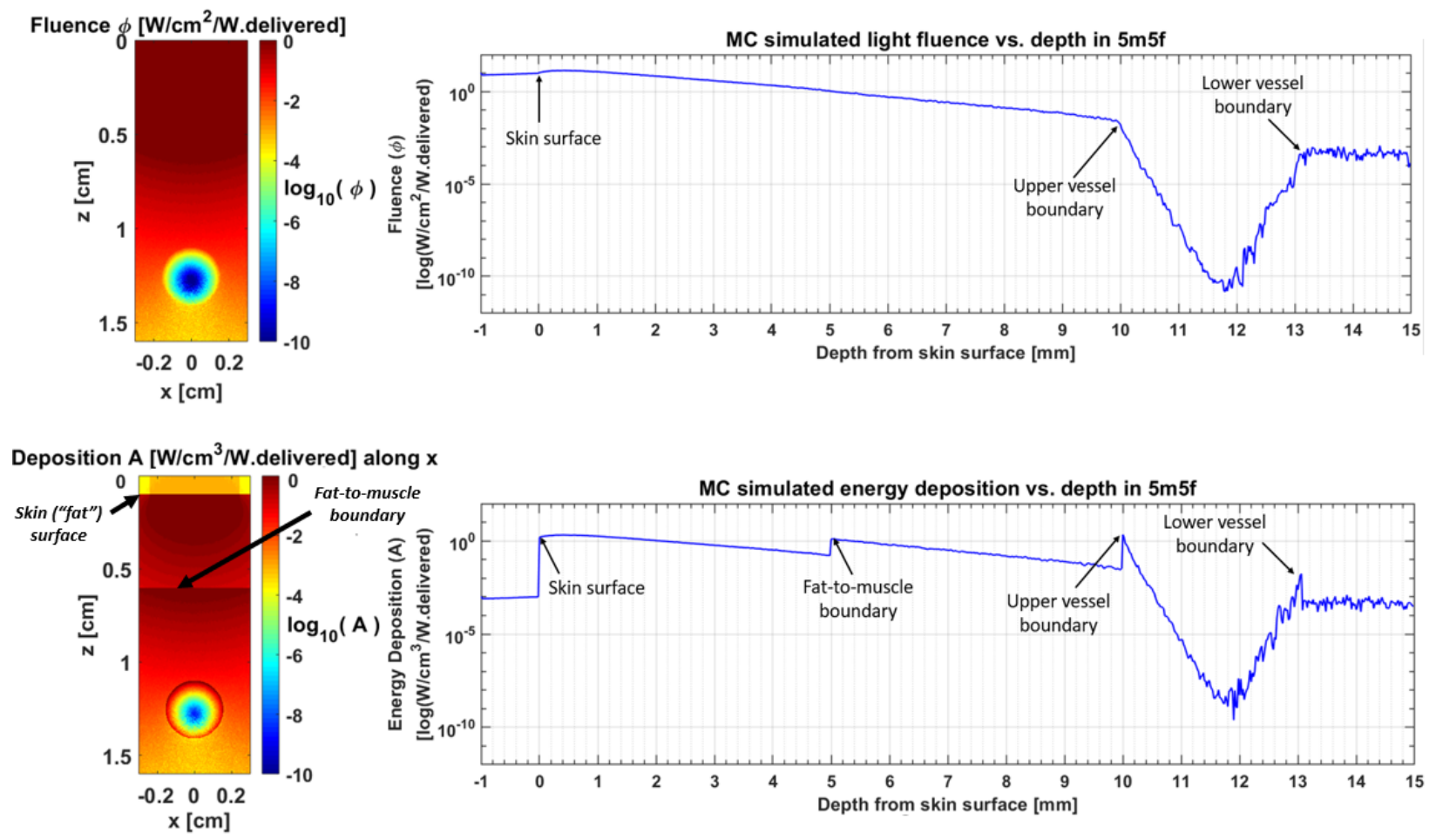

Normalized MC simulated PA signal ampltiude vs.depth in $\mathbf{5 m 5 f}$

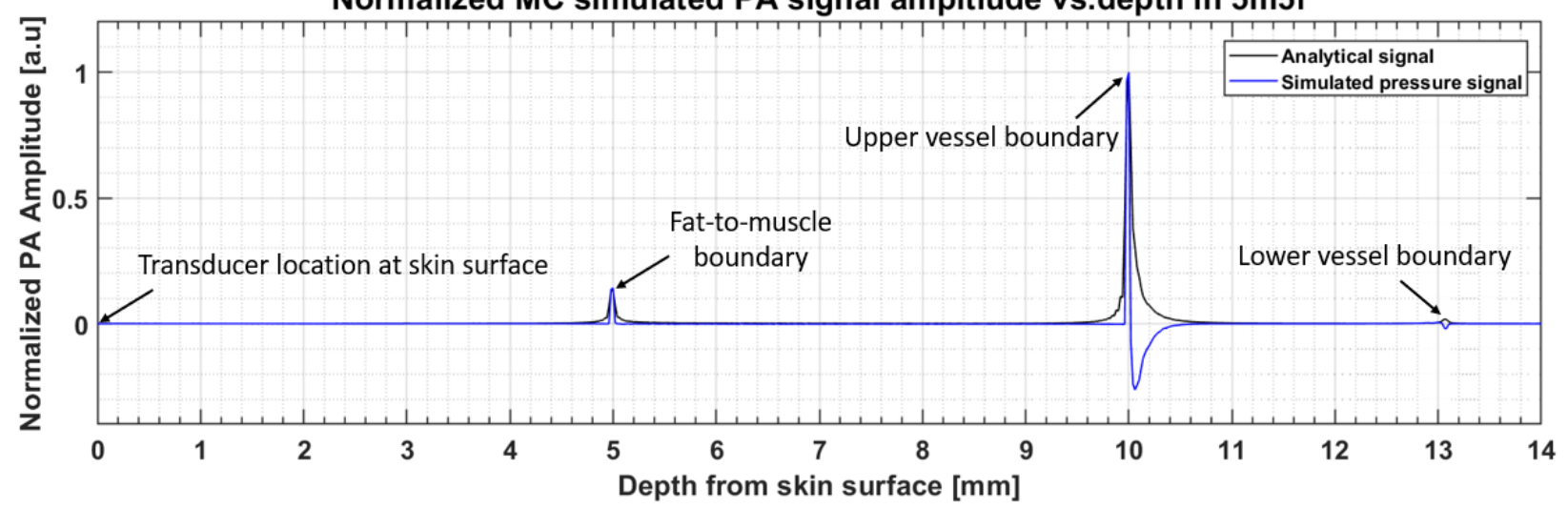

Figure 3.4: TOP-MC simulation generated 2D light light fluence distribution and the corresponding line plot vs depth at $\mathrm{x}=0 \mathrm{~cm}$ in the $5 \mathrm{~m} 5 \mathrm{f}$ simulation. MIDDLE- MC simulation generated 2D light energy absorption distribution and the corresponding line plot vs depth at $\mathrm{x}=0 \mathrm{~cm}$ in the $5 \mathrm{~m} 5 \mathrm{f}$ simulation. BOTTOM-MC generated PA signal amplitude vs. tissue depth at $\mathrm{x}=0 \mathrm{~cm}$. 

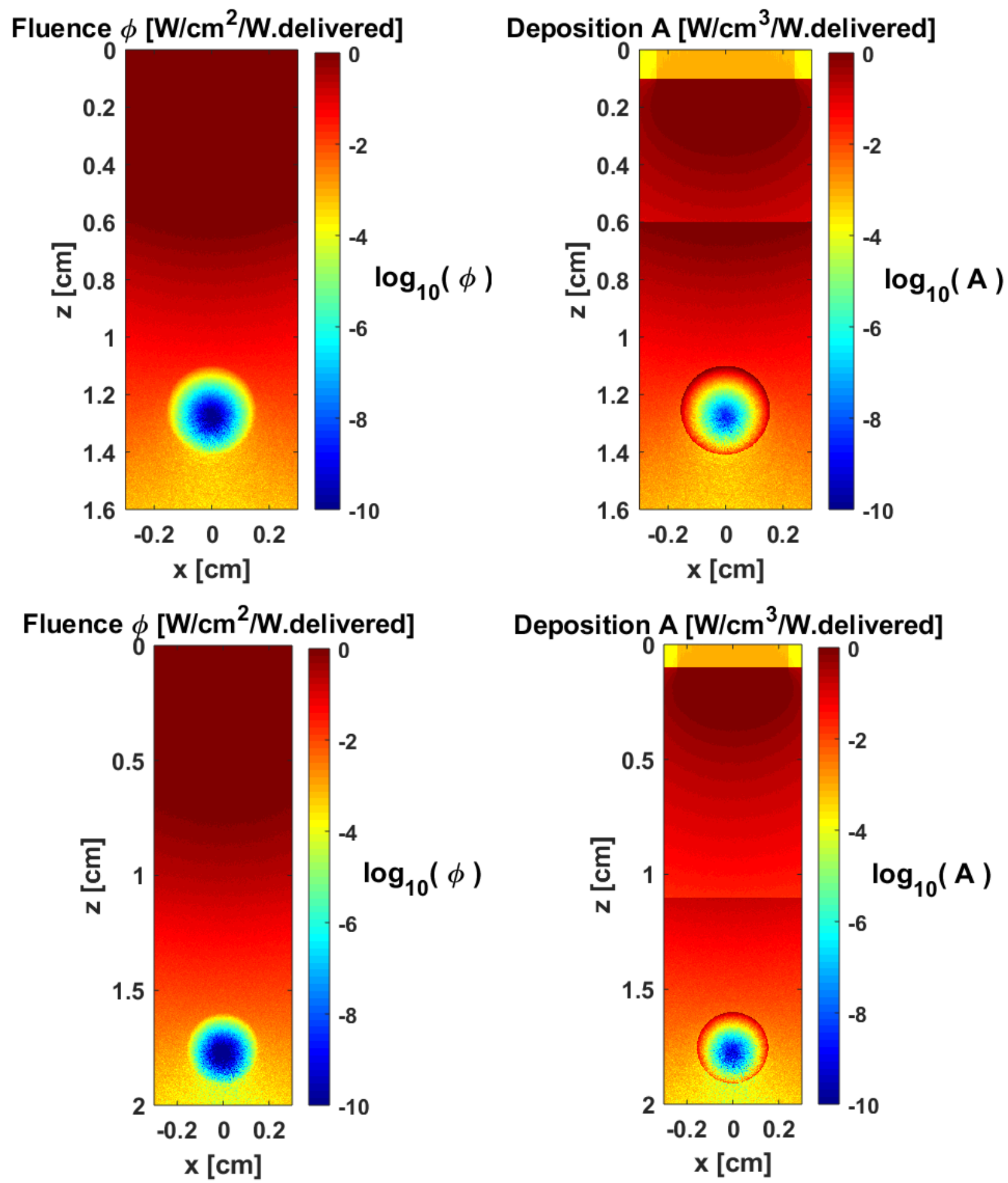

Figure 3.5: The MC generated 2D distribution profiles of light fluence (LEFT) and energy deposition (RIGHT) for the 5m5f (TOP) and 5m10f (BOTTOM) simulations. 

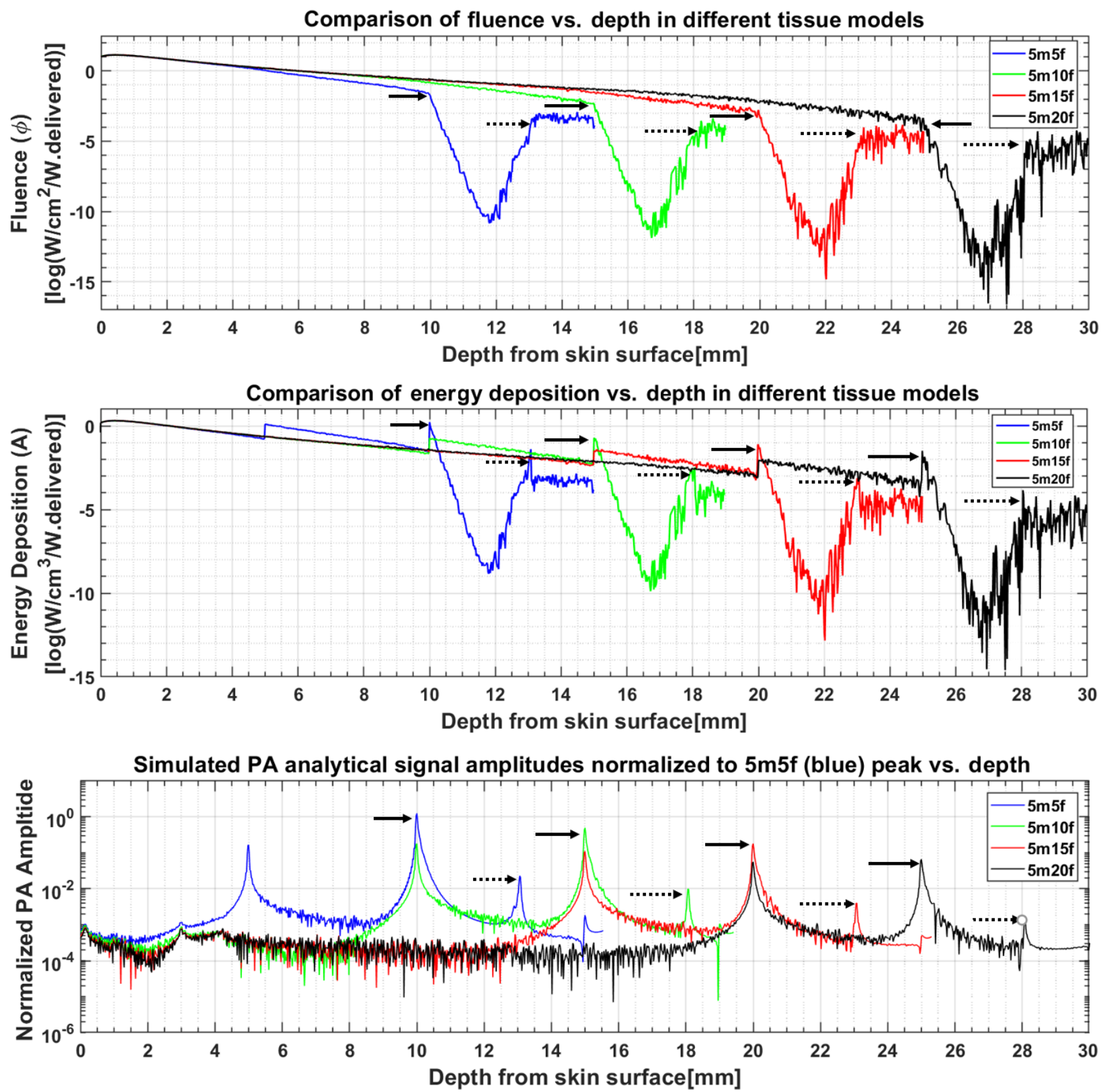

Figure 3.6: TOP- Fluence vs. tissue depth, MIDDLE- Energy deposition vs. tissue depth and BOTTOM- Normalized PA amplitude vs. tissue depth for the $5 \mathrm{~m} 5 \mathrm{f}, 5 \mathrm{~m} 10 \mathrm{f}, 5 \mathrm{~m} 15 \mathrm{f}$ and $5 \mathrm{~m} 20 \mathrm{f}$ tissue models. All measurements are taken at $\mathrm{x}=0 \mathrm{~cm}$. The solid arrows mark the positions of the top vessel boundary (muscle-to-vessel) where most of the energy is deposited, while the dotted arrows mark the positions of the bottom vessel boundary (vessel-to-muscle) where relatively smaller energy and PA peaks are observed. 


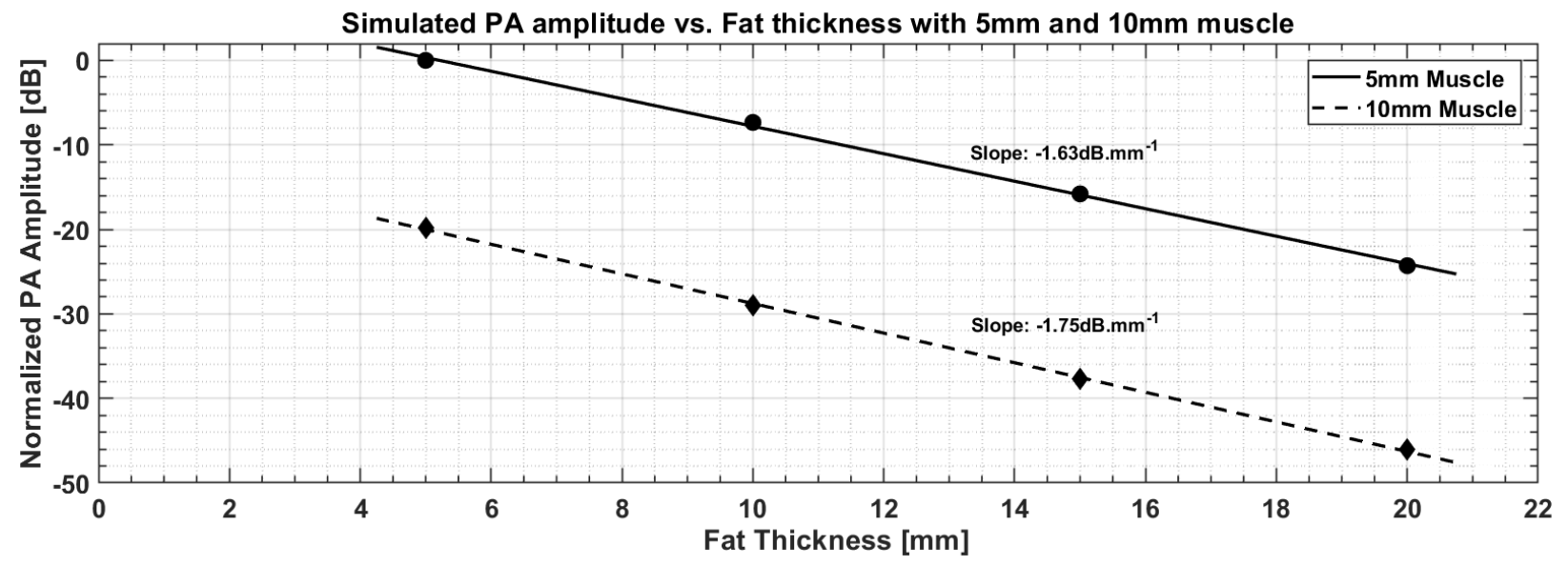

Figure 3.7: MC simulated attenuation of peak PA amplitude(dB) normalized to the maximum in $5 \mathrm{~m} 5 \mathrm{f}$. 


\subsection{Experimental Measurements}

Figure 3.8 shows two representative 2D B-mode PA images in a 10m5f (left) and a $5 \mathrm{~m} 20 \mathrm{f}$ (right) phantom. As in the MC simulations, the strongest signals are observed at the boundaries. The strongest signal is observed at the phantom surface and vessel boundary, due to the strong in the $5 \mathrm{~m} 20 \mathrm{f}$ phantom is due to lower fluence relative to the fat/muscle boundary in the $10 \mathrm{~m} 5 \mathrm{f}$ phantom.

Figure 3.9 shows a a total of six sample experimental measurements of PA amplitude. For the 5m5f, 5m10f and 5m15f phantoms with MetHb absorptions of $\mu_{a}=90 \mathrm{~cm}^{-1}$ (red) and $\mu_{a}=175 \mathrm{~cm}^{-1}$ (blue). The largest PA signal peak is observed at at phantom surface. The target region of interest lays within the vessel between 6 and $9 \mathrm{~mm}$, particularly at the upper vessel boundary at $\sim 7 \mathrm{~mm}$ to $\sim 9 \mathrm{~mm}$. Given the relatively strong absorption of MetHb within the vessel, not enough light reaches the lower boundary, where a second peak is potentially expected. A low peak is observed at the fat-to-muscle boundary in the $5 \mathrm{~m} 5 \mathrm{f}$ phantom at a depth of $\sim 13-14 \mathrm{~mm}$ or $\sim 5-6 \mathrm{~mm}$ below the surface. The SNR at the vessel detected with a $175 \mathrm{~cm}^{-1}$ MetHb sample are higher than with the $90 \mathrm{~cm}^{-1}$, and both decrease with increasing phantom thickness given the increased light attenuation.

The measured peak SNRs as defined in Chapter 2 are listed in Table 3.3. The values in the parenthesis represent the number $n$ defined in Chapter 2 as the maximum number of SNR values averaged to achieve a coefficient of variation $C V \leq 10 \%$. Figure $\mathbf{3 . 1 0}$ shows plots of SNR vs. Fat Thickness and MetHb absorption. The top plot, SNR vs. MetHh absorption extrapolates the SNRs for different phantoms (5m5f, 5m10f, 5m15f, 5m20f, 10m5f, 10m10f) to the range of desired MetHb absorptions expected in an IPH, i.e. $\mu_{a}$ between $310 \mathrm{~cm}^{-1}$ and $518 \mathrm{~cm}^{-1}$. SNRs at these absorption values are used to estimate a best linear fit for SNR vs. Fat thickness for $\mu_{a}=310 \mathrm{~cm}^{-1}$ and $518 \mathrm{~cm}^{-1}$ in the bottom plot (black lines). The extrapolated maximum fat thickness for either absorption is found to be $33 \mathrm{~mm}$ and 


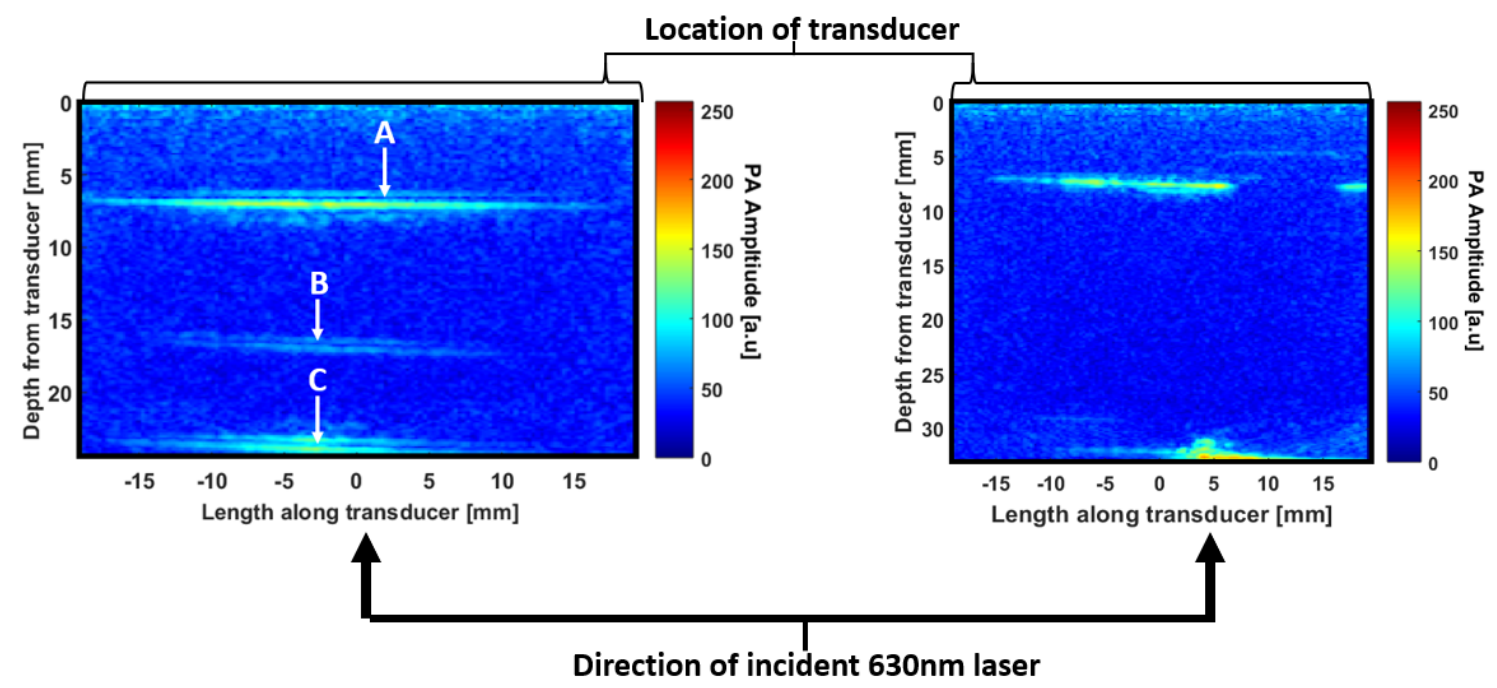

Figure 3.8: Representative B-mode (2D) PA images in a 10m5f (left) and 5m20f (right) phantoms. A- Signal form MetHb at the vessel boundary. B- Signal from the fat/muscle boundary. C-Signal from the phantom surface. Note the absence of a discernible signal at fat/muscle boundary in the $5 \mathrm{~m} 20 \mathrm{f}$ phantom on the right.

$38 \mathrm{~mm}$ for $\mu_{a}=310 \mathrm{~cm}^{-1}$ and $518 \mathrm{~cm}^{-1}$ respectively. The lines $\mathbf{A}, \mathbf{B}, \mathbf{C}, \mathbf{D}, \mathbf{E}$ are best fits to the experimental data with absorptions $\mu_{a}=175 \mathrm{~cm}^{-1}, 132 \mathrm{~cm}^{-1}, 90 \mathrm{~cm}^{-1}, 30 \mathrm{~cm}^{-1}$ and $12 \mathrm{~cm}^{-1}$. Table 3.4 lists the slope of the lines A, B, C, D, E and their respective linear fitted slope, standard deviation of the slope and R-squared value. The average slope $(-1.45 \pm 0.07) d B . m^{-1}$ describes the attenuation of SNR in fat independent of MetHb absorption. Note that is does not properly account for tissue acoustic attenuation.

\begin{tabular}{|c|c|c|c|c|c|}
\hline \multirow{2}{*}{ Phantom } & \multicolumn{5}{|c|}{ Absorption coefficient $\left(\mathrm{cm}^{-1}\right)$ of injected MetHb } \\
\cline { 2 - 6 } & $\mathbf{1 2}$ & $\mathbf{3 0}$ & $\mathbf{9 0}$ & $\mathbf{1 3 2}$ & $\mathbf{1 7 5}$ \\
\hline $\mathbf{5 m 5 f}$ & $31.11(38)$ & $35.12(28)$ & $43.00(38)$ & $51.00(22)$ & $54.75(28)$ \\
\hline $\mathbf{5 m 1 0 f}$ & $23.09(58)$ & $28.56(28)$ & $37.19(100)$ & $40.93(83)$ & $48.48(68)$ \\
\hline $\mathbf{5 m 1 5 f}$ & $16.94(88)$ & $17.50(50)$ & $35.04(10)$ & $34.54(100)$ & $42.18(19)$ \\
\hline $\mathbf{5 m 2 0 f}$ & & & $27.31(100)$ & & $32.85(93)$ \\
\hline $\mathbf{1 0 m 5 f}$ & & & $25.27(100)$ & & $39.47(100)$ \\
\hline $\mathbf{1 0 m 1 0 f}$ & & & $22.28(100)$ & & $33.00(100)$ \\
\hline
\end{tabular}

Table 3.3: Averaged experimentally measured peak SNR(dB) for different phantoms and MetHb absorptions. The values in parentheses define the maximum number of peak SNRs per measurement averaged to achieve a coefficient of variation $C V \leq 10 \%$. The blank table cells indicate an absence of valid measurement. 

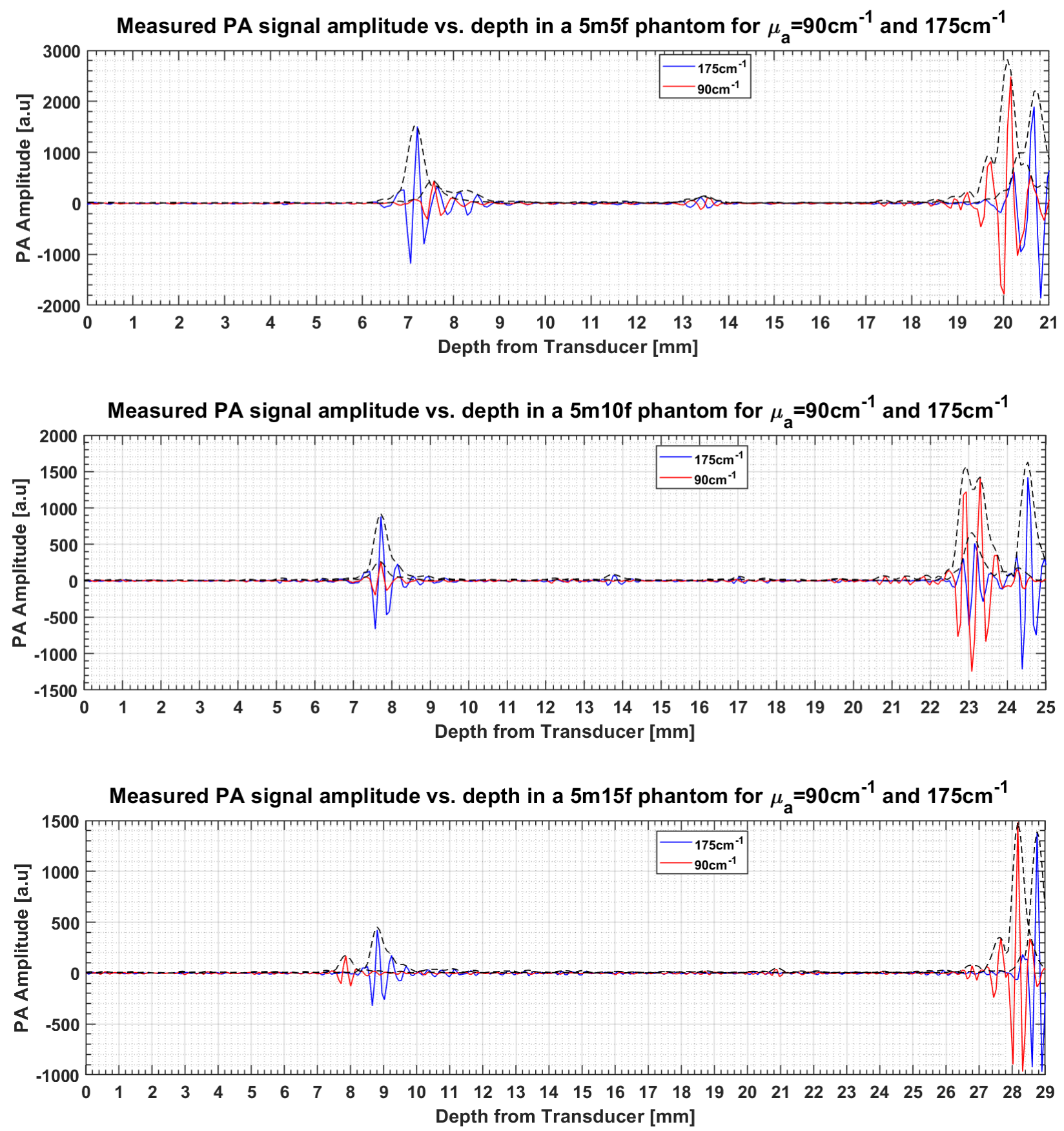

Figure 3.9: Representative examples of experimental measurements of PA RF lines in 5m5f (top), $5 \mathrm{~m} 10 \mathrm{f}$ (middle) and $5 \mathrm{~m} 15 \mathrm{f}$ (bottom) phantoms with injected metHb samples with absorption coefficients of $90 \mathrm{~cm}^{-1}$ (RED) and $175 \mathrm{~cm}^{-1}$ (BLUE). 

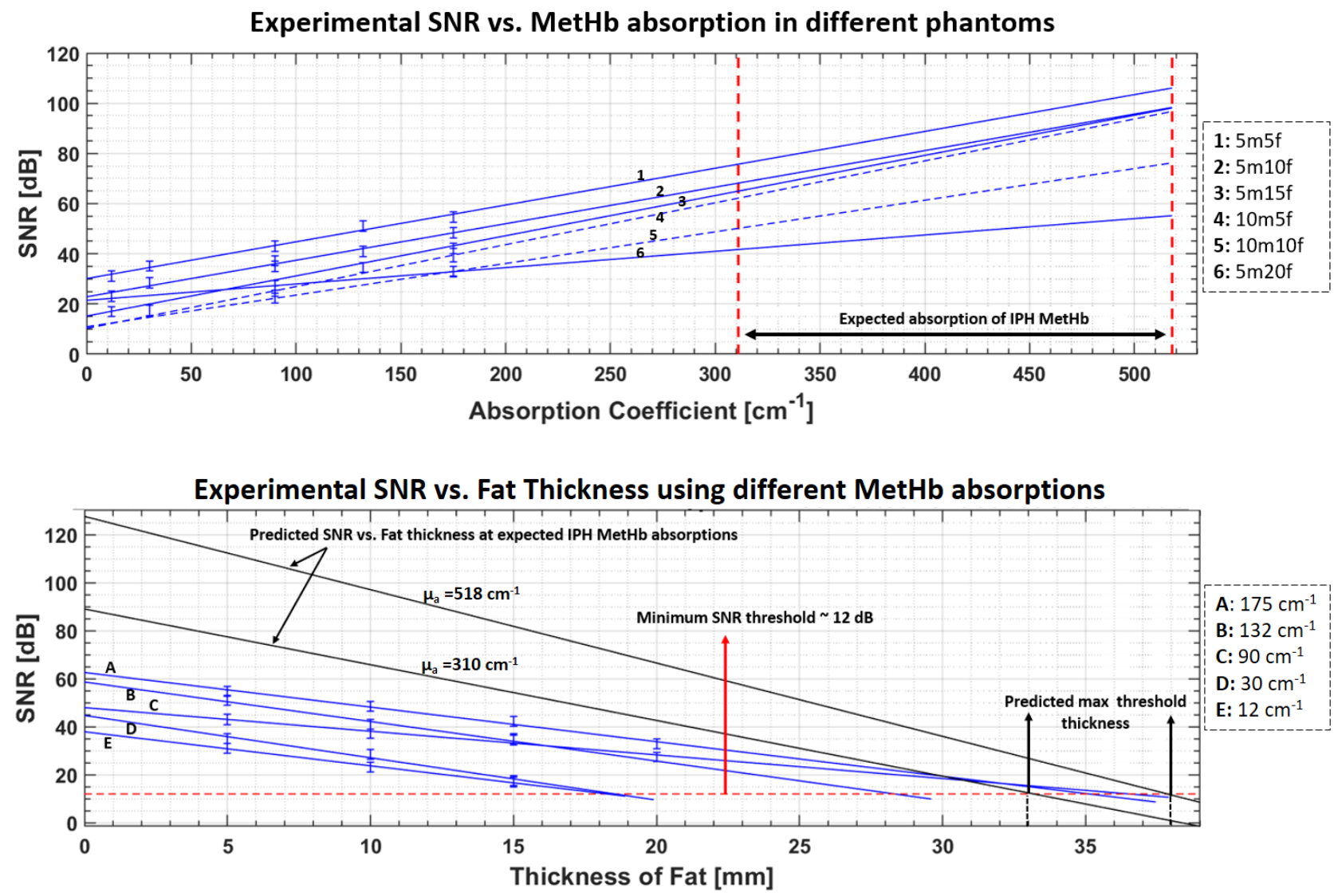

Figure 3.10: TOP- Measured SNR(dB) vs. measured MetHb absorption. The red lines define the range of MetHb absorptions in IPH. BOTTOM- Measured SNR(dB) vs. fat thickness for a fixed muscle thickness of $5 \mathrm{~mm}$, with varying injected MetHb absorption strengths. The red line defines the SNR threshold for detection.

\begin{tabular}{|c|c|c|c|}
\hline $\begin{array}{l}\text { Absorption coefficent } \\
\text { of MetHb }\left(\mathrm{cm}^{-1}\right)\end{array}$ & $\begin{array}{c}\text { Slope } \\
\left(\mathrm{dB} \cdot \mathrm{mm}^{-1}\right)\end{array}$ & $\begin{array}{c}\text { Standard Deviation } \\
\text { of Slope }\end{array}$ & R-squared \\
\hline 12 & -1.42 & 0.108 & 0.994 \\
\hline 30 & -1.76 & 0.260 & 0.979 \\
\hline 90 & -0.984 & 0.144 & 0.959 \\
\hline 132 & -1.65 & 0.213 & 0.984 \\
\hline 175 & -1.44 & 0.106 & 0.989 \\
\hline
\end{tabular}

Table 3.4: The attenuation of SNR with fat thickness given by the experimental slopes in dB. $\mathbf{m} \mathbf{m}^{-\mathbf{1}}$ for different MetHb absorptions. 


\section{Chapter 4}

\section{Discussion}

\subsection{Assumptions and Limitations}

The results in the previous chapter demonstrate an exponential attenuation of simulation calculations and experimental measurements of SNR. This is evident from the linear slopes in the logarithmic plots of SNR vs. Fat thickness in Figures 3.7 and 3.10. The magnitude of the simulation SNR attenuation in fat was calculated to be $1.63 \mathrm{~dB} . \mathrm{mm}^{-1}$ and $1.75 \mathrm{~dB} . \mathrm{mm}^{-1}$ in $5 \mathrm{~mm}$ and $10 \mathrm{~mm}$ of muscle, respectively. The magnitude of the experimental measurement

was $1.45 \mathrm{~dB} . \mathrm{mm}^{-1}$. The lower experimental measurement relative to simulation is expected to be lower given the setup of acoustic detection; simulation calculations had a detector located at the skin surface, resulting in an increasing source-to-detector distance with increasing tissue thickness, leading to signal decay as a result of the inverse-square law. This is in contrast to the experimental setup, where the source-to-detector distance was fixed at $\sim 7-9 \mathrm{~mm}$ for all phantoms.

Measured SNR values between $42 d B$ and $65 d B$ were predicted at the average carotid depth of $\sim 20 \mathrm{~mm}$ given IPH MetHb absorptions between $310 \mathrm{~cm}^{-1}$ and $518 \mathrm{~cm}^{-1}$, with an SNR up to $12 d B$ expected at depths up to 38 and $\sim 43 \mathrm{~mm}$ in the that absorption range. 
It is important to note that neither simulation nor experimental models properly account for acoustic attenuation. The simulations assumed negligible acoustic attenuation, while the experimental phantoms had an attenuation of $\sim 0.2 d B . \mathrm{mm}^{-1}$ at $5 \mathrm{Mhz}[75]$. As such, this chapter discusses the results presented in the previous chapter in the context of relevant experimental and/or theoretical assumptions and limitations, and applies relevant corrections to improve the accuracy of the experimental and theoretical predictions.

\subsubsection{Tissue properties}

The tissues models used in the MC simulations and experimental phantoms are simplifications of biological tissue. The skin's topmost layer, the epidermis was not explicitly modeled. Despite being relatively thin $(60-100 \mu m)$, the epidermis does have substantial optical absorption [36]. This is due primarily to the presence of melanin. Melanin has strong absorption within the UV-VIS range[36], and is the substance responsible for giving skin, hair and eyes their color. Higher levels of melanin results in darker skin pigmentation, and hence different skin types (tones) have different absorption. The second tissue type not explicitly modeled in the MC simulations and experimental phantoms is the carotid artery wall. The thickness of the carotid artery wall is $\sim 1-2 \mathrm{~mm}$ and is assumed to have an optical absorption similar

to the aorta where $\mu_{a} \sim 0.52 \mathrm{~cm}^{-1}$ [76,77]. Including both tissue types would result in lower SNR measurements for a given depth and MetHb concentration.

\subsubsection{Plaque, IPH and MetHb}

The tissue models assume a $3.1 \mathrm{~mm}$ vessel. The actual diameter of the carotid ranges between $4-7 \mathrm{~mm}$ [78]. Non-IPH plaque components have negligible absorption at $630 \mathrm{~nm}$ relative to MetHb, and are therefore neglected; the vessel model is assumed to be entirely filled with MetHb. The model also assumes the IPH is located at the wall closer to the light source. 
The target absorption of MetHb ranging between $310 \mathrm{~cm}^{-1}$ and $518 \mathrm{~cm}^{-1}$ were calculated using the MRIIPH in-vivo reported MetHb concentrations [23]. This calculation assumes a linear relationship between absorption and concentration [79]. There is no consideration for any saturation effects arising from approaching high chromophore concentrations, wherein PA signal strength plateaus with increasing chromophore concentration [80].

\subsubsection{Acoustic Attenuation, Noise and Detection}

\subsubsection{Acoustic attenuation}

The MC and experimental tissue models do not explicitly model acoustic tissue properties, most importantly, acoustic attenuation. Acoustic attenuation is both medium (tissue type) and frequency dependent, at about $\alpha_{F a t} \sim 0.063 \mathrm{~dB} \cdot \mathrm{mm}^{-1} \mathrm{MHz} z^{-1}$ and $\alpha_{M u s c l e} \sim$ 0.33dB. $\mathrm{mm}^{-1} \mathrm{MHz}^{-1}$ [78]. Given the central frequency of the linear L7-4 transducer used is $\sim 5 M h z$, this gives $\alpha_{f a t} \sim 0.315 \mathrm{~dB} . \mathrm{mm}^{-1}$ and $\alpha_{\text {Muscle }} \sim 1.65 \mathrm{~dB} . \mathrm{mm}^{-1}$. The acoustic

attenuation of the experimental phantoms is $\alpha_{\text {phantom }} \sim 0.2 d B . \mathrm{mm}^{-1}$ [75], while simulation models had negligible attenuation. As such the measured SNR values are overestimated, and the resulting SNR vs. Fat attenuation slopes are underestimated.

\subsubsection{Detector location}

The theoretical PA signal simulation assumes a point-like single-element detector at the central axis $(\mathrm{x}=0 \mathrm{~cm})$ on top the skin surface with negligible acoustic attenuation. The experimental measurements use a 128-element transducer, with element having a a width $\sim 0.3 \mathrm{~mm}$, placed at the bottom behind the target vessel at a fixed distance of $\sim 9 \mathrm{~mm}$ in all phantoms. The varying acoustic depths in the PA simulations result in increasing acoustic attenuation with increasing tissue depth. This effect is lost in the experimental measurements, as vessel-to-transducer distance is fixed for all tissues, which underestimates the SNR vs. Fat attenuation values measured. 


\subsubsection{Anatomical noise and artifacts}

Noise and artifacts due to anatomical structures were not explicitly considered in the simulation and experimental models. Acoustic waves generated from strongly absorbing structures can undergo strong acoustic reflections from hyperechoic anatomical structures, leading to increased noise and possible artifacts $[82,83]$. This is especially relevant given that the tissue models did not account for the epidermis. Given its strong absorption, especially in dark skin types, the epidermis would generate secondary acoustic waves, effectively making it a secondary ultrasound transmitter. While PA imaging is relatively speckle-free, speckle is inherent in ultrasound imaging and typically leads to degradation of boundaries in images (e.g. vessel boundary) [83,84]. The secondary acoustic generation and reflection from different anatomical structures such as the epidermis can lead to acoustic clutter and ultimately degrade signal and/or image SNR.

\subsubsection{Detection limits}

The SNR detection limit of $12 \mathrm{~dB}$ is taken to be an estimate of the signal SNR required for the imaging detection of IPH using PA. IPH detectability in imaging would depend on many factors such as IPH size, imaging device spatial and contrast resolution and noise (see 4.1.3.3 above). It should be noted that SNR calculations in this thesis are applied to signals (RF lines) and not images. The peak signal amplitude used for SNR calculations represents peak pixel intensity in an image.

The models used ignored detectability limits from geometric factors such as IPH size. Detection of an IPH is limited to only the parts of the IPH undergoing light-excitation; the model assumes complete illumination -and excitation- of an IPH target 


\subsubsection{Laser setup}

The OPO laser used was a single $630 \mathrm{~nm}$ beam with a spot size diameter of $\sim 4.8 \mathrm{~mm}$ with pulse width of $4 \mathrm{~nm}$ to $6 \mathrm{~nm}$, and a power output ranging between $20-25 \mathrm{~mW}$, or $111-138 \mathrm{~mW} \cdot \mathrm{cm}^{-2}$. With a repetition rate of $10 \mathrm{~Hz}$, this equates to an radiant energy fluence $H_{e}$ between $11.1 \mathrm{~mJ} . \mathrm{cm}^{-2}$ and $13.8 \mathrm{~mJ}_{\mathrm{cm}}^{-2}$. The exposure times per measurement ranged between 5 and 10 seconds. According to the the American National Standard Institute (ANSI), the above specs and exposure times limit the Maximum Permissible Exposure (MPE) to between $6.3 \mathrm{~mJ} . \mathrm{cm}^{-2}$ and $7.5 \mathrm{~mJ} . \mathrm{cm}^{-2}$ for 10 and 5 second-long exposures, respectively [85]. For exposure times of 5 seconds, the MPE $7.5 \mathrm{mJ.cm} \mathrm{cm}^{-2}$ is between 1.48 and 1.84 times less than the experimental energy fluence outputs. Given the linearity between PA pressure generation and fluence from $p_{0}=\Gamma \mu_{a} F(\vec{r})$, we predict experimental SNR values to be lower by at least a factor between $20 \log (1.48)=3.4 d B$ and $20 \log (1.84)=5.3 d B$ if $\mathrm{MPE}$ limits were considered.

\subsection{Corrections for Maximum Imaging Depth}

The measured concentrations of IPH MetHb predict absorption coefficients ranging between $310 \mathrm{~cm}^{-1}$ and $518 \mathrm{~cm}^{-1}$. Figure 3.10 shows the SNR vs. Fat thickness lines for both MetHb concentrations (black) down to the $12 d B$ threshold (dotted red). This gives an estimated maximum fat thickness between 33 and $38 \mathrm{~mm}$. Considering the $5 \mathrm{~mm}$ of muscle already present, the projected maximum imaging depth is between $38 \mathrm{~mm}$ and $43 \mathrm{~mm}$. No similar prediction was done for 10mm-muscle phantoms due to a lack of sufficient data points; only two MetHb absorptions for two fat thicknesses.

The main factors meriting correction are epidermal optical attenuation, carotid wall optical attenuation and tissue (muscle and fat) acoustic attenuation, all which would lead to measurement of lower SNR. 


\subsubsection{Optical attenuation corrections}

Epidermal absorption can be accounted for by approximating its effective absorption thickness -calculated by the product of the absorption coefficient by physical thickness $\mu_{a} \cdot t$ to an equivalent effective fat absorption thickness. The thickness of the epidermis is $t_{\text {Epidermis }}=$ $60 \mu m$ [53]. The absorption coefficient of fat is $\mu_{a-F a t}=0.15 \mathrm{~cm}^{-1}$. This allows for the calculation of an equivalent physical thickness $t_{F a t}=\frac{\mu_{a-E \text { Eidermis }} \cdot t_{\text {Epidermis }}}{\mu_{a-F a t}}$. Table 4.1 lists the range of epidermal absorptions in different skin types. Lightly pigmented skins (Type I and II) have a correction equating to an physical fat thickness of $2 \mathrm{~mm}$ to $8 \mathrm{~mm}$, whereas for darkly-pigmented skins (Types $\mathrm{V}$ and VI) the equivalent fat thickness is from $23 \mathrm{~mm}$ to $54 \mathrm{~mm}$. Carotid wall absorption correction is carried out using a similar approach used for epidermal absorption correction. The carotid wall's absorption is approximated by that of the aorta, with $\mu_{a} \sim 0.52 \mathrm{~cm}^{-1}$ at $633 \mathrm{~nm}$ or $\sim 630 \mathrm{~nm}$ [77]. Given the carotid wall thickness ranges between $1.13 \mathrm{~mm}$ and $1.58 \mathrm{~mm}$ [69], the equivalent fat thickness is $3.9 \mathrm{~mm}$ to $5.8 \mathrm{~mm}$.

Applying both epidermal and carotid wall corrections, any given fat thickness $\mathbf{t}_{\mathbf{f}}$ can approximated to a $1.13 \mathrm{~mm}$-thick carotid wall in addition to $60 \mu \mathrm{m}$ epidermis and an equivalent fat thickness $\mathbf{t}_{\mathbf{e q}}=\mathbf{t}_{\mathbf{f}}-\mathbf{2}-\mathbf{3 . 9} \mathbf{m m}$ with the $2 \mathrm{~mm}$ and $3.9 \mathrm{~mm}$ being the lower-end corrections for epidermal and carotid-wall optical accentuation, respectively. For example a 25mm-thick $5 \mathrm{~m} 20 \mathrm{f}$ phantom would approximate to a $\sim 20$-thick phantom consisting of 1.1-mm carotid wall, 5mm-muscle, $14.1 \mathrm{~mm}$-fat and a $60 \mu \mathrm{m}$-epidermis.

Note that the above corrections only consider optical attenuation of the epidermis and carotid artery wall. They do not consider other effect such as secondary photosensitive waves generated by the epidermis. 


\begin{tabular}{|l|c|c|}
\hline \multicolumn{1}{|c|}{ Skin Type } & $\begin{array}{c}\text { Absorption of } \mathbf{6 0} \boldsymbol{\mu} \mathbf{m} \\
\text { epidermis } \mathbf{c m}^{-1}\end{array}$ & $\begin{array}{c}\text { Maximum Imaging } \\
\text { Depth (mm) }\end{array}$ \\
\hline Lightly-pigmented & 4 to 20 & 32 \\
\hline Moderately-pigmented & 35 to 51 & 26 \\
\hline Darkly-pigmented & 57 to 135 & 9 \\
\hline
\end{tabular}

Table 4.1: Different skin types with the corresponding $60 \mu \mathrm{m}$ epidermis absorption and predicted maximum imaging depth. Per the Fitzpatrick scale, lightly-pigmented skins are referred to as types I and II , moderately-pigmented as types III and IV and darkly-pigmented as types $\mathrm{V}$ and VI. The epidermal absorption values were reproduced from [53].

\subsubsection{Acoustic attenuation corrections}

Acoustic attenuation was not properly accounted for in the MC simulations and experimental measurements. The MC simulations assumed no acoustic attenuation, while the experimental phantoms had an acoustic attenuation $\sim 0.2 \mathrm{~dB} . \mathrm{mm}^{-1}$ at $5 \mathrm{MHz}$. The primary sources of acoustic attenuation in tissue are fat and muscle tissues, with acoustic coefficients at 5Mhz of $\alpha_{\text {Fat }}=0.315 \mathrm{~dB} \cdot \mathrm{mm}^{-1}$ and $\alpha_{\text {Muscle }} \sim 1.67 \mathrm{~dB} \cdot \mathrm{mm}^{-1}$ [67]. Given the small thickness and attenuation of the epidermis, and the small acoustic attenuation of arterial wall, their contribution was neglected.

The acoustic attenuation of the experimental phantoms is $\sim 0.2 d B . \mathrm{mm}^{-1}$ [71]. This is lower than the fat and muscle attenuation, and given the fixed vessel-to-transducer distance of $\sim$ $8 \mathrm{~mm}$, the phantom produces constant acoustic attenuation of $\mathbf{0 . 2} \mathbf{d B} \cdot \mathbf{m} \mathbf{m}^{\mathbf{- 1}} \cdot \mathbf{8} \mathbf{m m}=\mathbf{1 . 6} \mathbf{d B}$.

The SNR vs. Fat thickness plots (Figure 3.10) are for a fixed $5 \mathrm{~mm}$ of muscle. This results in constant muscle acoustic attenuation of $\alpha_{\text {Muscle }}=1.67 \mathrm{~dB} \cdot \mathrm{mm}^{-1} \cdot \mathbf{5 m m}=8.35 \mathrm{~dB}$. The fat-dependent attenuation of SNR is equal to the sum of the slope of the SNR vs. Fat lines and the fat acoustic attenuation of $\alpha_{\text {Fat }}=\mathbf{0 . 3 1 5} \mathrm{dB} \cdot \mathbf{m m}^{-\mathbf{1}}$. The average slope calculated from Table 3.4 is $-1.45 \mathrm{~dB} . \mathrm{mm}^{-1}$. Accounting for fat's acoustic attenuation, the total fat-dependent SNR attenuation is $-(1.45+0.315) d B . m^{-1}=1.765 d B . m^{-1}$. The total attenuation experienced and and SNR would then be $\alpha_{\text {Total }}=1.765 \mathrm{~dB} . \mathrm{mm}^{-1}+8.35 \mathrm{~dB}$. 
To estimate a corrected maximum imaging depth, must apply $\alpha_{\text {Total }}$ to an an attenuated signal, i.e. the phantom's attenuation of $1.6 d B$ is neglected. For example, consider the $518 \mathrm{~cm}^{-1}$ line in bottom plot of Figure 3.10. To estimate the maximum imaging depth we solve for thickness of fat in $\mathbf{m m}$ in:

$$
12 d B=-(3.03+0.315) d B . m m^{-1}+(127+1.6-8.35) d B
$$

where on the LHS, $12 d B$ is the detection threshold. On the RHS, $-3.03 d B . m^{-1}$ is the slope of the $518 \mathrm{~cm}^{-1}$ line, $-0.315 \mathrm{~dB} \cdot \mathrm{mm}^{-1}$ the fat acoustic attenuation, $+127 \mathrm{~dB}$ the $\mathrm{y}$-intercept value, $+1.6 d B$ the correction for phantom acoustic attenuation and $-8.35 d B$ the muscle acoustic attenuation. The estimated maximum fat thickness is calculated to be $\sim 32 \mathrm{~mm}$. Accounting for the already $5 \mathrm{~mm}$ of muscle, the total depth is $37 \mathrm{~mm}$. Applying the same approach to the $310 \mathrm{~cm}^{-1}$ line gives an estimated total depth of $\sim 32 \mathrm{~mm}$. Finally, epidermal and carotid wall corrections equate to at least $5 \mathrm{~mm}$ of fat for Type I skin, i.e. the maximum imaging depth would be $5 \mathrm{~mm}$ less: $27 \mathrm{~mm}$ to $32 \mathrm{~mm}$. Table 4.1 lists the different skin types with the corresponding epidermis absorption and predicted maximum imaging depth.

Applying the same acoustic and optical attenuation corrections for SNRs measured at the average carotid depth of $\sim 20 \mathrm{~mm}$, we get an SNR of $50 \mathrm{~dB}$ for a MetHb absorption of $518 \mathrm{~cm}^{-1}$.

\subsection{Simulation vs. Experiment}

The Monte Carlo and experimental measurements of signal amplitude versus increasing tissue thickness demonstrate an exponential decay of SNR, as evidenced by the negative linear slopes in the logarithmic plots in Figures 3.7 and 3.10. This behavior is expected and in line with the Beer-Lambert Law of exponential light attenuation [65]. 
The measured SNR vs. Fat thickness slope ranges between $-0.98 \mathrm{~dB} / \mathrm{mm}$ (for $\mu_{a}=90 \mathrm{~cm}^{-1}$ ) and $-1.76 \mathrm{~dB} . \mathrm{mm}^{-1}$ for $\left(\mu_{a}=30 \mathrm{~cm}^{-1}\right)$, with an average of $-1.45 \mathrm{~dB} / \mathrm{mm}$ measured over 5 MetHb absorptions with varying fat thickness given a fixed $5 \mathrm{~mm}$ of muscle. Table 4.2 lists the SNR vs. Fat thickness slopes calculated in the Monte Carlo based simulations compared to experimental results. The errors listed are the standard deviation of the best fit slope. The magnitude of the experimental slope of $1.45 \mathrm{~dB} . \mathrm{mm}^{-1}$ for $5-\mathrm{mm}$ muscle phantoms is smaller than the simulation slope of $1.63 \mathrm{~dB} \cdot \mathrm{mm}^{-1}$. This is due to the experimental setup of a fixed vessel-to-transducer distance as SNR attenuation effects due to the inverse-square are neglected.

However, we suspect that the the slope of $-0.98 \mathrm{~dB} \cdot \mathrm{mm}^{-1}$ for $\mu_{a}=90 \mathrm{~cm}^{-1}$ might be a outlier, as it is over 6 standard deviations away from the mean. More measurements over a wider array of absorptions are warranted to bolster the claim, however. If the outlier is ignored, the observed average slope over 4 MetHb absorptions is $(-1.57 \pm 0.08) \mathrm{dB} . \mathrm{mm}^{-1}$, which is closer to the simulations results. Finally, applying acoustic attenuation corrections to the simulation and experimental slopes gives a simulation attenutation of $(1.63+0.315) d B . \mathrm{mm}^{-1}=$ $1.945 \mathrm{~dB} . \mathrm{mm}^{-1}$ and an experimental slope of $1.765 \mathrm{~dB} . \mathrm{mm}^{-1}$. If the outlier is ignored, it would be $1.885 \mathrm{~dB} \cdot \mathrm{mm}^{-1}$.

The purpose of the simulations were to provide a qualitative study of SNR vs. tissue (fat) thickness. Given a reference experimental SNR (e.g. 5m5f), the simulation results allow us to predict experimental SNR with increasing tissue thickness. Though comparable, the difference between corrected simulation and experimental attenuation values of $1.945 \mathrm{~dB} . \mathrm{mm}^{-1}$ and $1.765 \mathrm{~dB} . \mathrm{mm}^{-1}$ (or $1.885 \mathrm{~dB} . \mathrm{mm}^{-1}$ if the outlier is dropped) indicate a need for more rigorous simulations and experimental setup identical detector setup, and more experimental data points. 


\begin{tabular}{|c|c|c|}
\hline & Monte Carlo (dB/mm) of Fat & Experiment (dB/mm) of Fat \\
\hline $\mathbf{5} \mathbf{~ m m}$ Muscle & $-1.63 \pm 0.04$ & $-1.45 \pm 0.07$ \\
\hline $\mathbf{1 0} \mathbf{~ m m}$ Muscle & $-1.75 \pm 0.02$ & - \\
\hline
\end{tabular}

Table 4.2: The MC simulation and experimental calculations of the SNR attenuation in Fat tissue. The errors are the standard deviations for 4 data points per slope measurement.

Finally, we note that the experimental results discussed were produced using a $5 \mathrm{MHz}$ ATL L7-4 128-element linear transducer connected to a Vantage Verasonics $128^{\mathrm{TM}}$ system. The results are likely to vary between different transducers and systems. Stronger SNR can be expected with curved array transducers, for instance [42]. Higher frequency transducers would detect lower SNR as a result of higher acoustic attenuation experienced at higher frequencies [81]. 


\section{Chapter 5}

\section{Conclusions and Future Work}

\subsection{Thesis Conclusions}

The hypothesis presented in the opening chapter stated that PA techniques can be used for the imaging and detection of carotid IPH. To that end, the specific aims focused on developing tissue mimicking models, simulating light propagation, energy deposition, PA signal generation, and finally experimentally measuring PA signals generated from MetHb samples in tissue mimicking phantoms using a widely available ultrasound imaging device. The experimental results demonstrated that PAI can be potentially used for detecting carotid IPH. More specifically, in the context of assumptions, limitations and corrections discussed in Chapter 4, the experimental results demonstrated that using a commonly available 5 MHz L7-4 linear transducer, Verasonics system, and an in-vivo IPH MetHb concentration of $15 \mathrm{mmol} . \mathrm{L}^{-1}$, SNR values of $\sim 50 \mathrm{~dB}$ are possible at the average carotid depth of $\sim 20 \mathrm{~mm}$ in people with Type I skin. Furthermore, it was predicted in that IPH can be detected in depths ranging from $9 \mathrm{~mm}$ for dark-skin types to $32 \mathrm{~mm}$ for light-skin types (see Table 4.1).

Analysis of MR images of the neck helped determine the thickness and composition of the neck tissues. Published literature provided target optical properties of the tissue types used 
for tissue-mimicking models for experimental measurements and $\mathrm{MC}$ simulations. A total of eight neck tissue models of varying muscle and fat thicknesses (5m5f, 5m10f, 5m15f, 5m20f, 10m5f, 10m10f, 10m15f and 10m20f) were used in MC simulations of light propagation, energy deposition and PA signal generation from a MetHb target with absorption of $100 \mathrm{~cm}^{-1}$ - A total of 21 tissue-mimicking phantoms defining six different geometries were used for experimental measurements. The optical absorption and scattering of the phantoms were compared against target values, and verified with spectrophotometric measurements. Five MetHb absorptions were used for experimental measurements. While the desired MetHb absorptions between $310 \mathrm{~cm}^{-1}$ and $518 \mathrm{~cm}^{-1}, 5$ phantoms were made with absorptions between $12 \mathrm{~cm}^{-1}$ and $173 \mathrm{~cm}^{-1}$ to predict the maximum imaging depth for the desired absorption range. It was shown that detectable signals can be potentially detected up to depths of $27 \mathrm{~mm}$ and $32 \mathrm{~mm}$, assuming $\leq 5 \mathrm{~mm}$ of muscle. Furthermore, the experimental results demonstrated an average magnitude of fat-dependent SNR attenuation of $1.45 \mathrm{~dB} . \mathrm{mm}^{-1}$. This is comparable to the MC simulation theoretical value of $1.63 \mathrm{~dB} \cdot \mathrm{mm}^{-1}$. Accounting for acoustic attenuation, the values are $1.565 \mathrm{~dB} . \mathrm{mm}^{-1}$ and $1.94 \mathrm{~dB} . \mathrm{mm}^{-1}$ The theoretical calculation can help predict experimental SNR in thicker phantoms provided a measured SNR for some reference phantom is known, e.g. $5 \mathrm{~m} 5 f$. Note that the fixed source-to-transducer distances in the experimental setup potentially underestimate SNR attenuation. As such, we cautiously state that the predicted maximum imaging depth could be an overestimate.

Finally, the experimental results discussed were produced using a 5MHz ATL L7-4 128element linear transducer connected to a Vantage Verasonics $128^{\mathrm{TM}}$ system. The results are likely to vary between different transducers and systems. Stronger SNR can be expected with curved array transducers, for instance [42]. Higher frequency transducers would detect lower SNR as a result of higher acoustic attenuation experienced at higher frequencies [74]. 


\subsection{Future Directions}

The main scope of future work would focus on overcoming the limits presented in this thesis with the ultimate goal of creating as clinically relevant experimental and simulations procedures as possible. In that light, we suggest the following for future work:

1. Make use of superior computing capabilities or algorithms (e.g. CUDA parallel computing [86]) to improve the resolution in deep tissue simulations. This is pertinent to simulating optical propagation at large depths with high target absorption coefficient; stronger absorption requires higher resolution (smaller voxel sizes).

2. Utilize photoacostic phantoms that mimic both optical and acoustic tissue properties, while modeling as many tissue types as possible (epidermis of different skin types, dermis, fat, muscle, carotid artery wall and plaque).

3. Investigate the effects of practical limits of utilizing different laser geometries and setup (spot size, number of beams) on SNR. Larger spot sizes can potentially improve light fluence into deep targets [87]. The use of multiple beams irradiating the skin from different angles at different spots can also potentially improve fluence into deeper targets.

4. Conduct experimental PA measurements using stronger concentrations (absorptions) of MetHb that would ideally be withing the range of target concentrations found in carotid IPH in-vivo. Investigate the linearity of absorption (and PA amplitude) with MetHb concentration.

5. Utilize hybrid US-PA systems to mimic clinical measurement setups.

6. Conduct multi-spectral photoacoustic imaging by utilizing multiple laser wavelengths over the VIS-NIR range instead of just the 630nm used in the thesis. By capitalizing on multiple features unique to the MetHb absorption spectra (multiple peaks, slope, 
shape) a spectroscopic approach can be used for better specificity in identifying MetHb from among other chromophores. This can be investigated via Monte Carlo as well as experimental studies. 


\section{Appendix A}

\section{MR Images at the carotid bifurcation}

Table A.1 lists a range of patient ages and the corresponding measured depths of their left and right carotid bifurcation points. The average age was 65.7 age and the median was 65 yrs old. The average depth of both left and right carotid bifurcation points was found to be $(21.27 \pm 5.43) \mathrm{mm}$.

Figures A.1 and A.2 show some examples of the reference axial MR images of the carotid bifurcation point for 8 different subjects. The labeled distance define the the depth from the skin surface to the edge of the carotid artery. 


\begin{tabular}{|c|c|c|c|}
\hline & \multicolumn{3}{|c|}{ Depth of bifurcation point (mm) } \\
\hline Age (yrs) & Left & Right & Average \\
\hline 46 & 26 & 31 & 28.5 \\
\hline 52 & 27 & 21 & 24 \\
\hline 54 & 20 & 19 & 19.5 \\
\hline 57 & 29 & 32 & 30.5 \\
\hline 62 & 18 & 28 & 23.0 \\
\hline 65 & 19 & 21 & 21.5 \\
\hline 65 & 11 & 16 & 13.5 \\
\hline 65 & 19 & 21 & 20.0 \\
\hline 65 & 12 & 16 & 14.0 \\
\hline 65 & 18 & 23 & 20.5 \\
\hline 65 & 9 & 14 & 11.5 \\
\hline 70 & 20 & 20 & 20.0 \\
\hline 70 & 26 & 23 & 24.5 \\
\hline 70 & 21 & 20 & 20.5 \\
\hline N/A & 28 & 27 & 27.5 \\
\hline
\end{tabular}

Table A.1: A list of patient ages and the corresponding measured depths of their left and right bifurcation points using axial MR images at the carotid bifurcation point. The bottom age (N/A) was anonymized and does not reflect the actual age of the patient.
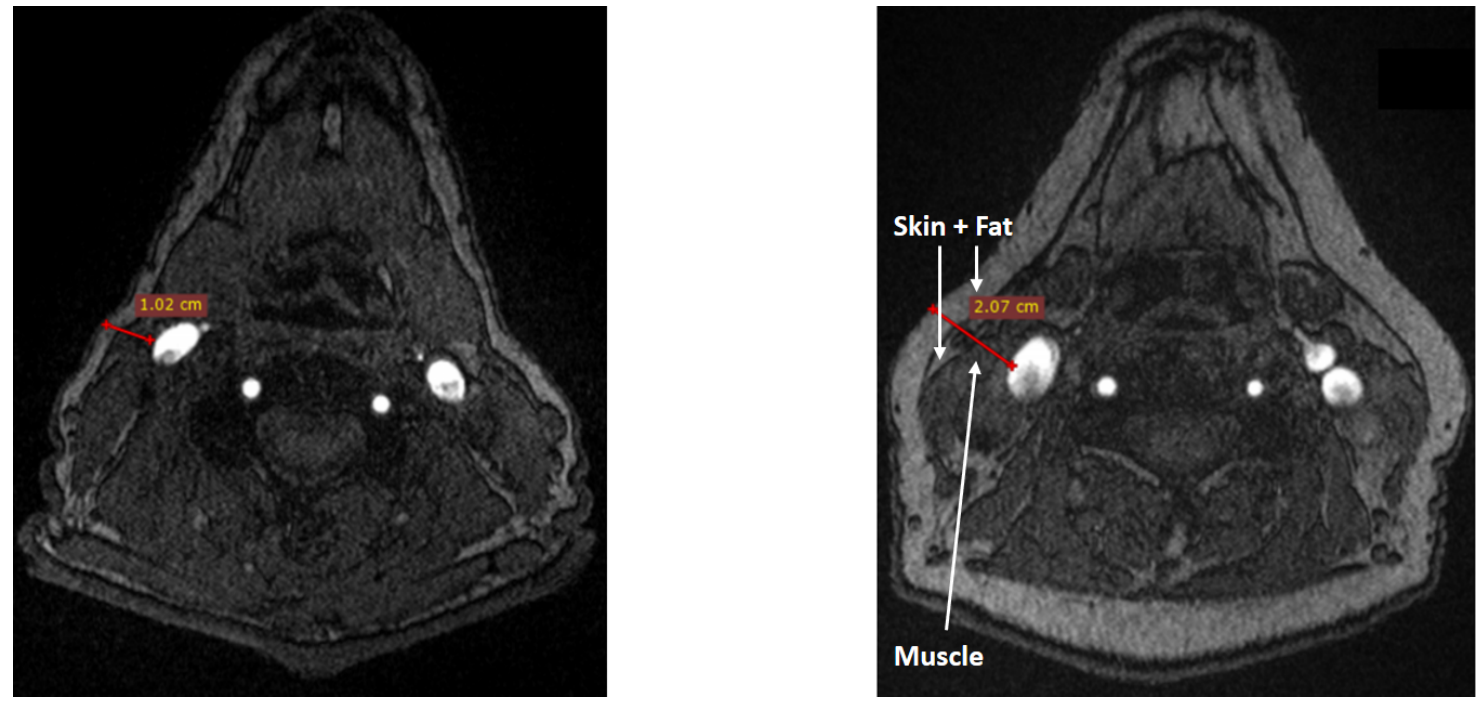

Figure A.1: Axial MR images at the carotid bifurcation point. 

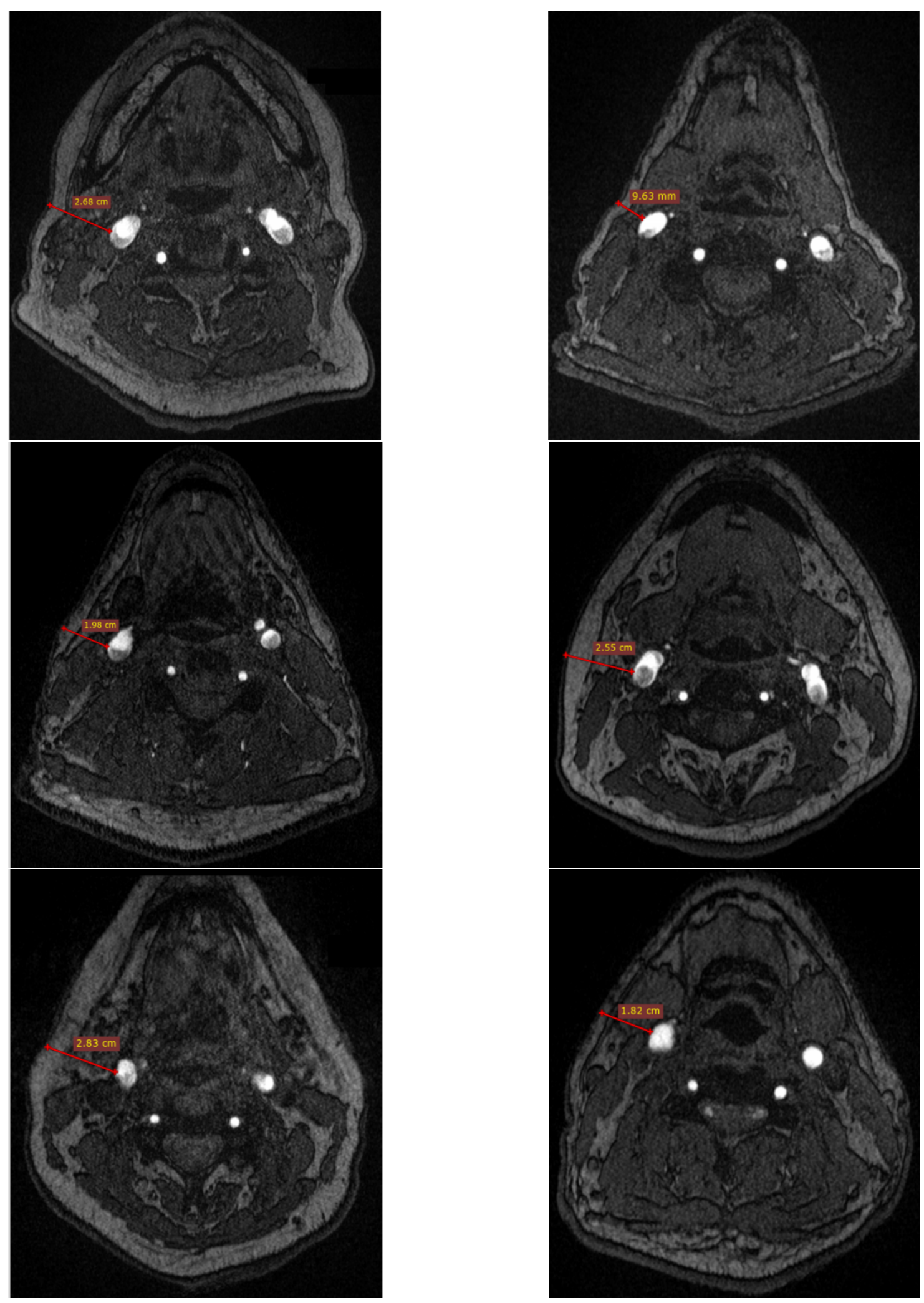

Figure A.2: Axial MR images at the carotid bifurcation point. 


\section{Appendix B}

\section{The Inverse Adding-Doubling Method}

The Inverse Adding-Double method, developed by Dr. Scott Prahl, takes spectrophotometric measurements of Total Trasmittence and Diffuse Reflectance to calculate to determine the optical properties of a homogeneous slab of material [64-67]. Parameters such as optical scattering, optical absorption and anisotropy can be determined via iteratively solving the radiaitve transport equation (RTE) until the calculated Transmittance and Reflectance match the observed values. The RTE describes the transport of light in a medium that absorbs, scatters and emits radiation, and can be defined as follows [67]:

$$
(\hat{s} \cdot \nabla) L(\boldsymbol{r}, \hat{s})=-\mu_{t} L(\boldsymbol{r}, \hat{s})+\mu_{s} \int_{4 \pi} P\left(\hat{s}, \hat{s}^{\prime}\right) L\left(\boldsymbol{r}, \hat{s}^{\prime}\right) d \omega^{\prime}
$$

where $L(\boldsymbol{r}, \hat{s})$ defines the radiance or specific intensity in units of energy per area per solid angle $W . \mathrm{cm}^{-2} s r^{-1}$ in the position denoted by $\boldsymbol{r}$ and direction specified by the unit vector $\hat{s}$ [67]. The total attenuation coefficient $\mu_{t}$ is the sum of both the absorption coefficient and scattering coefficient coefficients, $\mu_{a}$ and $\mu_{s}$ respectively [67]:

$$
\mu_{t}=\mu_{a}+\mu_{s}
$$

The left hand side of B.1 $(\hat{s} \cdot \nabla) L(\boldsymbol{r}, \hat{s})$ defines the rate of change of radiance at point $\boldsymbol{r}$ in the 
direction of $\hat{s}$, whereas the phase function $P\left(\hat{s}, \hat{s}^{\prime}\right)$ on the right hand side defines the fraction of light scattered from the direction $\hat{s}^{\prime}$ to $\hat{s}$ which is normal to the solid-angles $d \omega^{\prime}$. Finally, the rate of change on the left gives the net change in radiance resulting from attenuation losses due to absorption and scattering, i.e. $-\mu_{t}$ and gains resulting from isotropic scattering into $\hat{s}$, i.e. : $\int_{4 \pi} P\left(\hat{s}, \hat{s}^{\prime}\right) L\left(\boldsymbol{r}, \hat{s}^{\prime}\right) d \omega^{\prime}$.

Normally, the adding-doubling method attempts to solve RTE to calculate Transmission and Reflection from known absorption and scattering coefficients. The inverse adding-doubling method works "inversely" in that $\mu_{a}$ and $\mu_{s}$ are approximated by iteratively making new estimates until the calculated transmittance and reflectance match the experimentally observed values. Figure B.1 shows a flow-chart of the IAD process, whereas Table B.1 lists the used-defined parameters used in the IAD software.

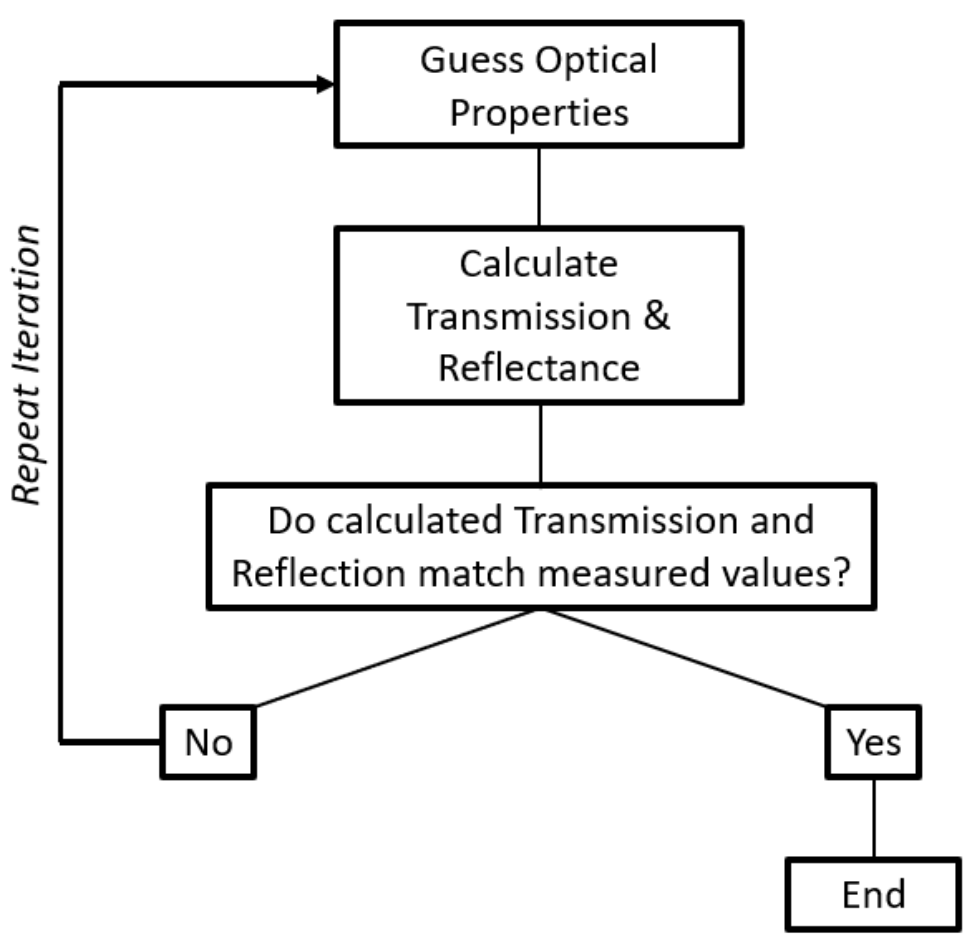

Figure B.1: Flowchart for IAD, modified from [67]. The method starts with an initial estimate of the the optical properties to calculate Transmission and Reflection values which are comparing the calculated with the measured values. The process is repeated iteratively with different optical properties until the calculated and measured transmission reflection values match. 


\begin{tabular}{|l|l|}
\hline \multicolumn{1}{|c|}{ Paramter } & \multicolumn{1}{c|}{ Value } \\
\hline Index of refraction of sample (Gelatin-based phantom) & 1.35 (measured) \\
\hline Index of refraction of the top and bottom slides (BK-7) & 1.5138 \\
\hline Thickness of sample [mm] & $2 \mathrm{~mm} \mathrm{to} \mathrm{3mm}$ \\
\hline Thickness of slides [mm] & 0 \\
\hline Diameter of illumination beam [mm] & 7.14 \\
\hline Reflectivity of the reflectance calibration standard & 0.98 \\
\hline Number of spheres used during each measurement & 1 \\
\hline Sphere diameter [mm] & 60 \\
\hline Sample port diameter [mm] & 18.75 \\
\hline Entrance port diameter [mm] & 16.74 \\
\hline Detector port diameter [mm] & 13.13 \\
\hline Reflectivity of the sphere wall & 0.98 \\
\hline Sphere diameter & 60 \\
\hline Number of Measurements & 2 \\
\hline
\end{tabular}

Table B.1: The parameter values used in the IAD software. No slides were used during the measurements -the edges of the samples were adhered to the wall of the integrating sphere's sample ports- and as such the thickness is defined as zero. 


\section{Appendix $\mathrm{C}$}

\section{Monte Carlo Simulation Results}

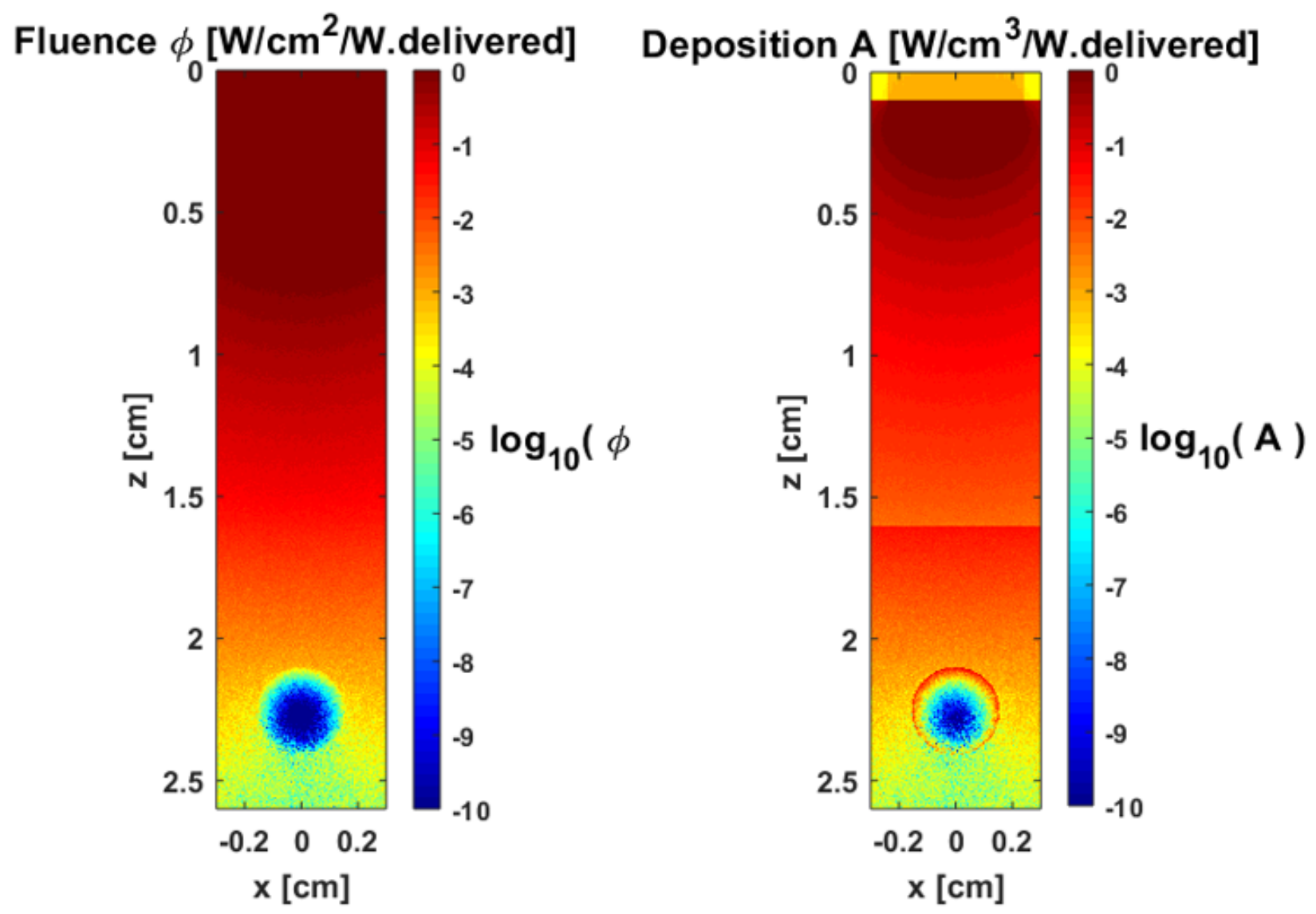

Figure C.1: 2D Fluence and Energy deposition profiles for the $5 \mathrm{~m} 15 \mathrm{f}$ simulation. 


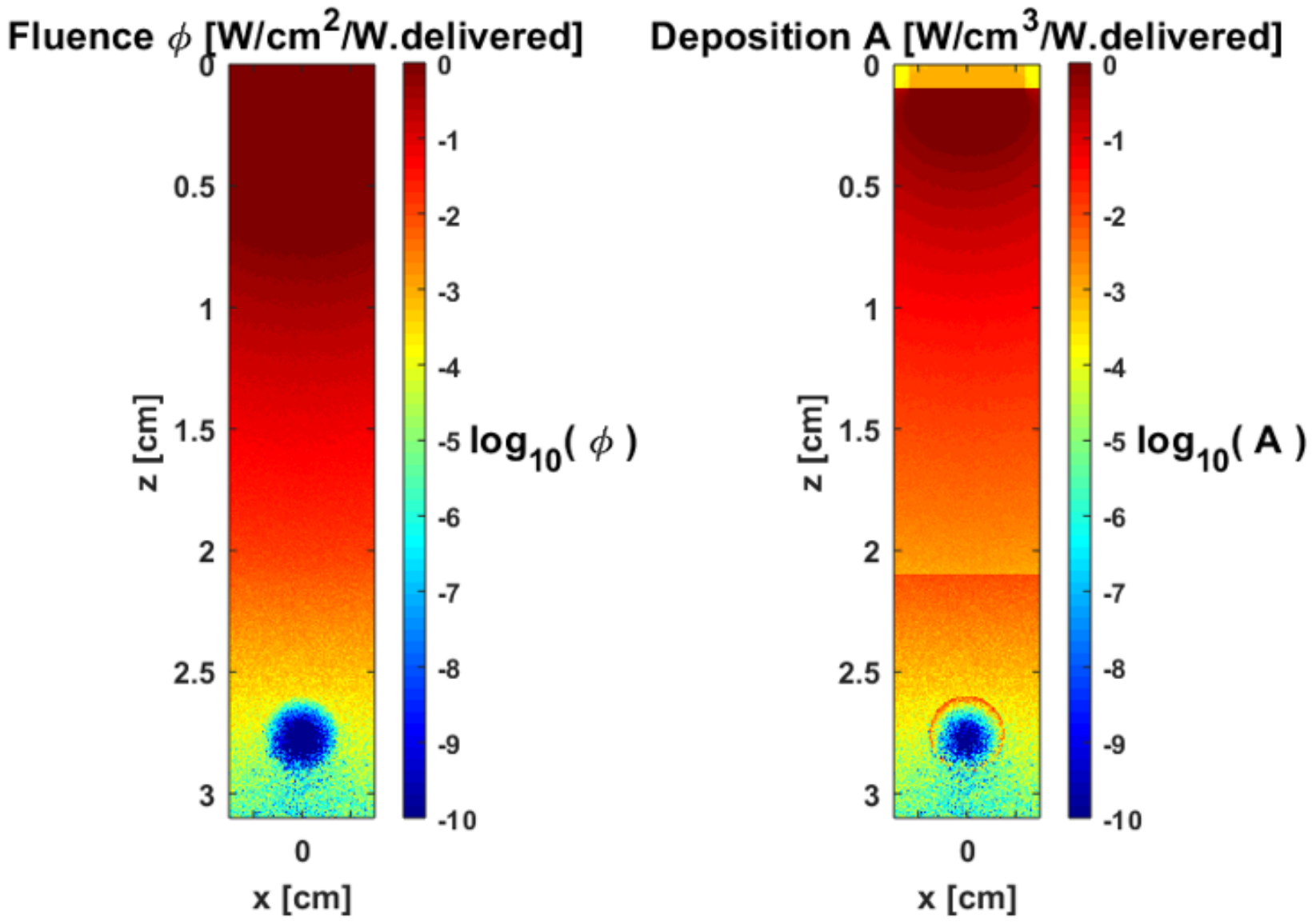

Figure C.2: 2D Fluence and Energy deposition profiles for the $5 \mathrm{~m} 20 \mathrm{f}$ simulation. 


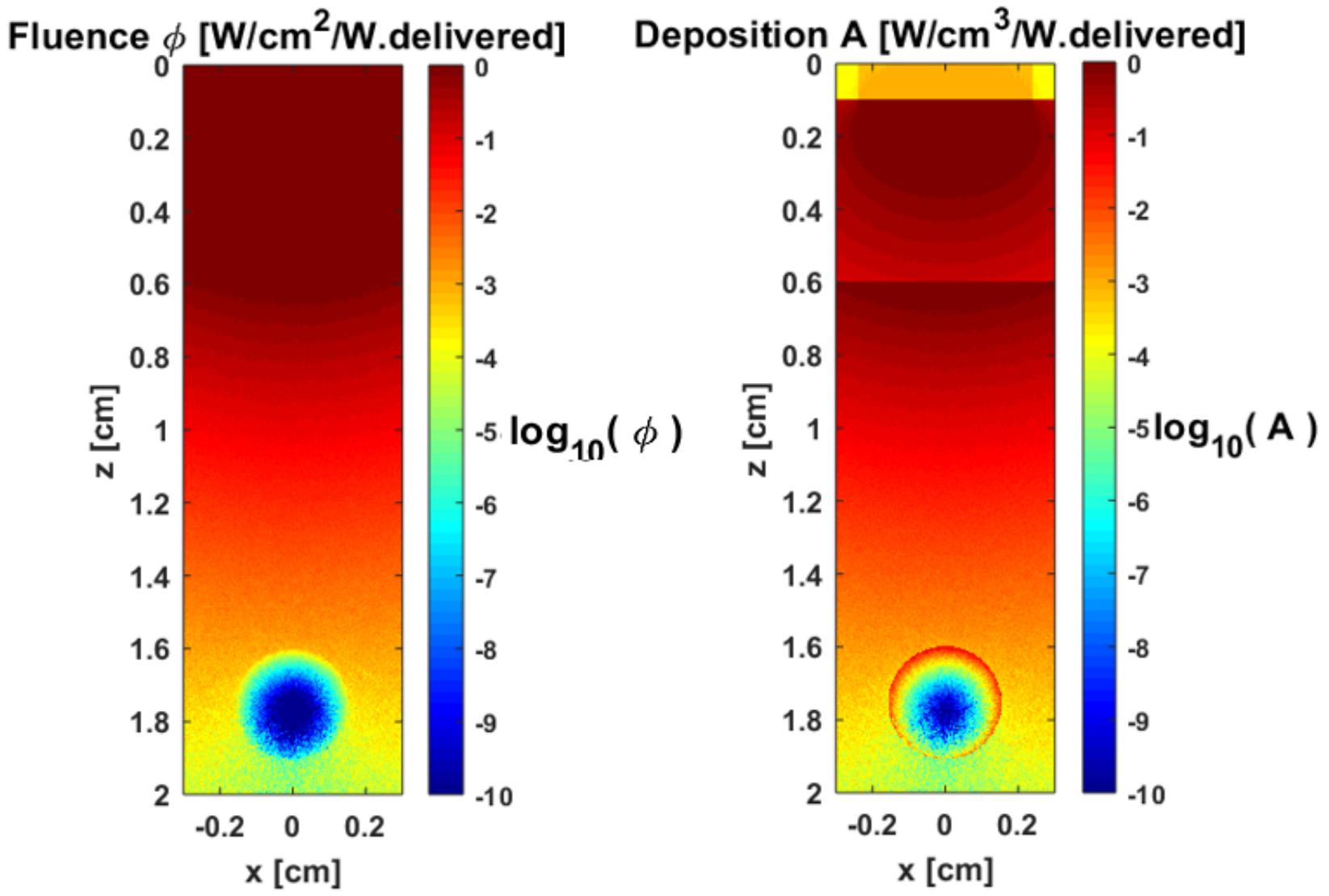

Figure C.3: 2D Fluence and Energy deposition profiles for 10m5f simulation. 


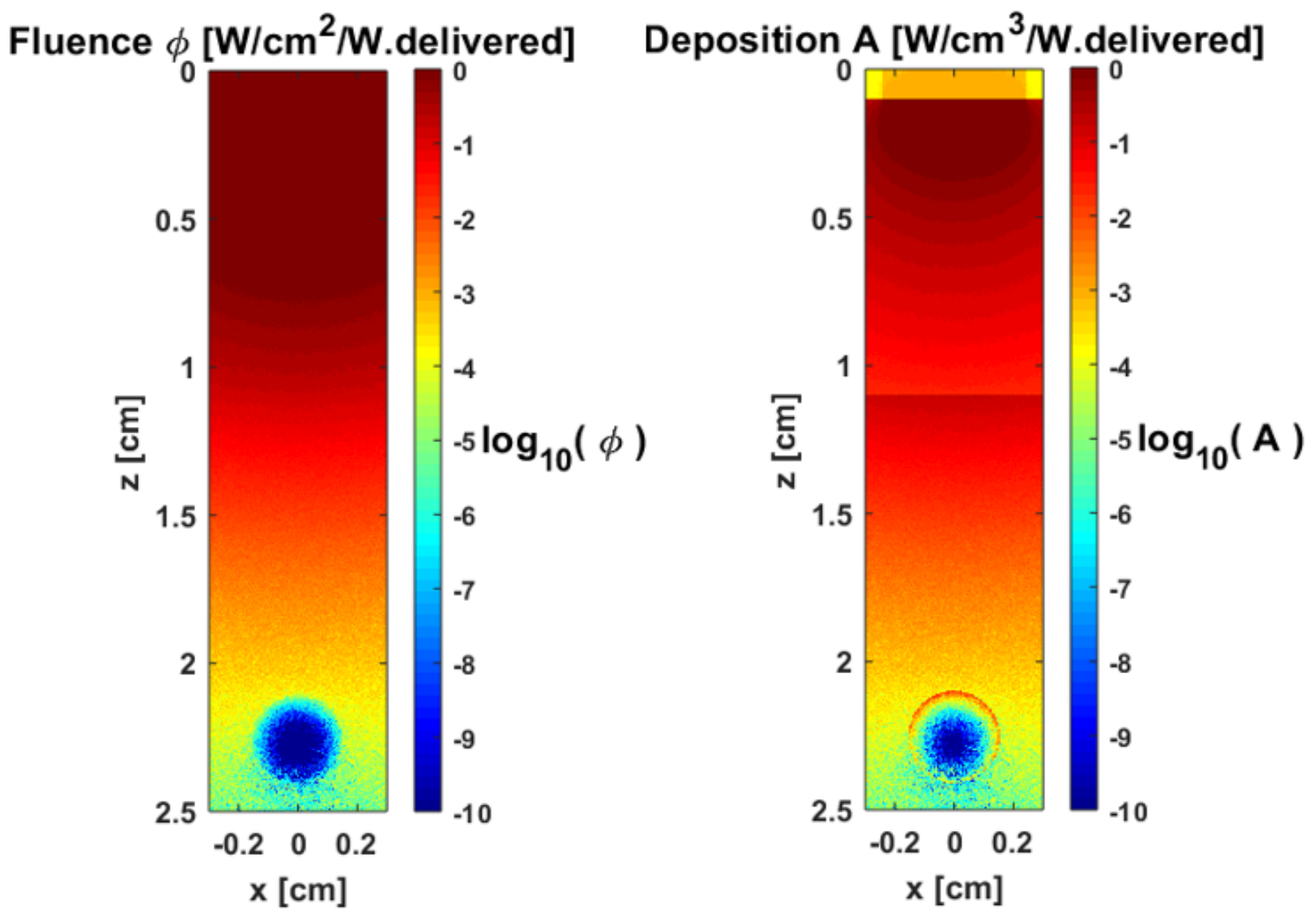

Figure C.4: 2D Fluence and Energy deposition profiles for the 10m10f simulation. 


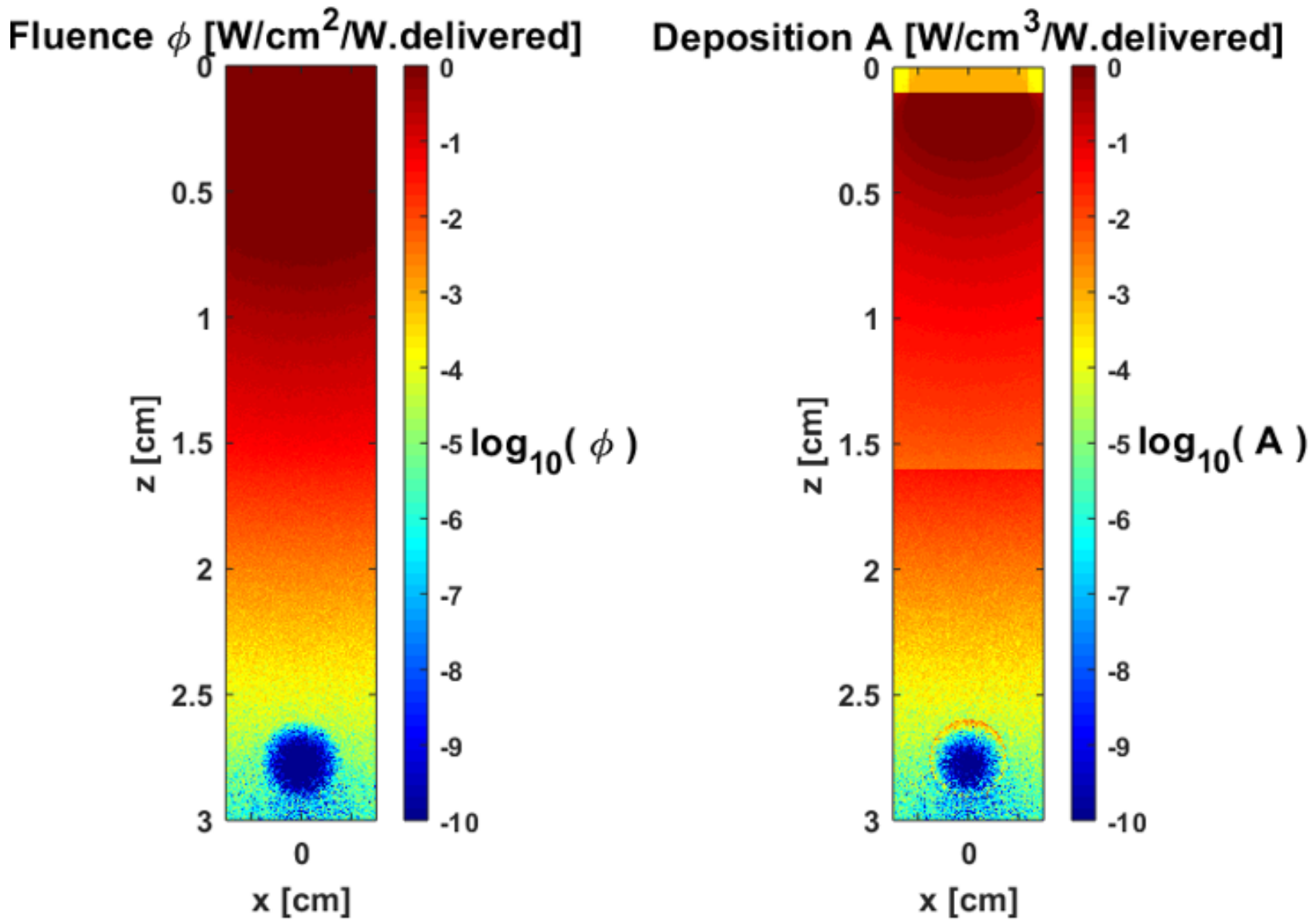

Figure C.5: 2D Fluence and Energy deposition profiles for the $10 \mathrm{~m} 15 \mathrm{f}$ simulation. 


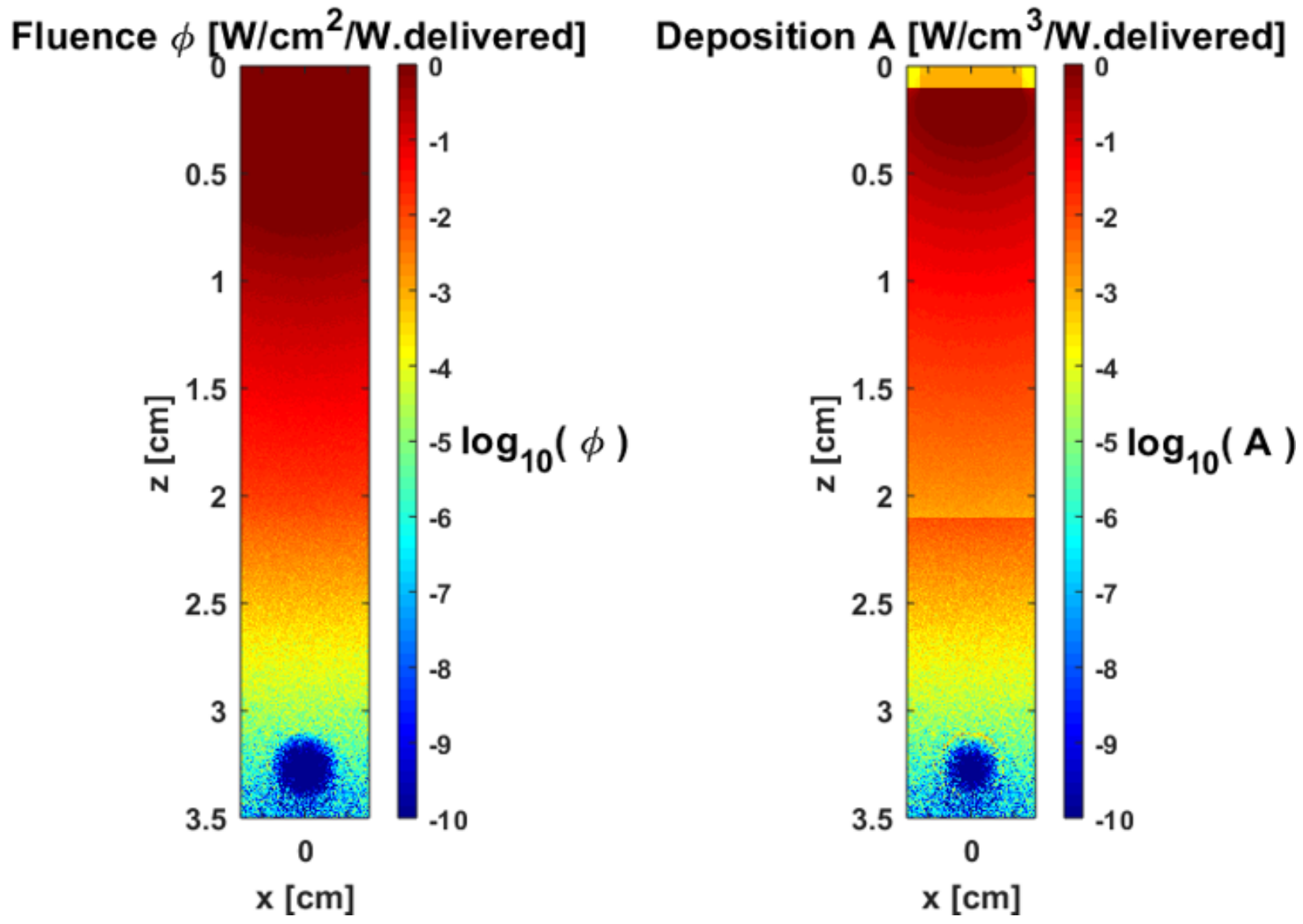

Figure C.6: 2D Fluence and Energy deposition profiles for the 10m20f simulation. 


\section{Appendix D}

\section{Sample measurements of TT and DR}

Figure D.1 plots the measured total transmittance (TT) and diffuse reflection (DR) spectra using the Shimadzu's UV-3600 UV-VIS-NIR spectrophotometer [].

TT spectra as in the top plot were used to estimate the absorption coefficient of the muscle

phantom: $\mu_{a}=-\frac{\ln (T T \%)}{d}$ where $d$ is the thickness of the phantom. TT and DR spectra as in the middle and bottom plots were used as inputs for the IAD method to estimate the reduced scattering coefficients. 

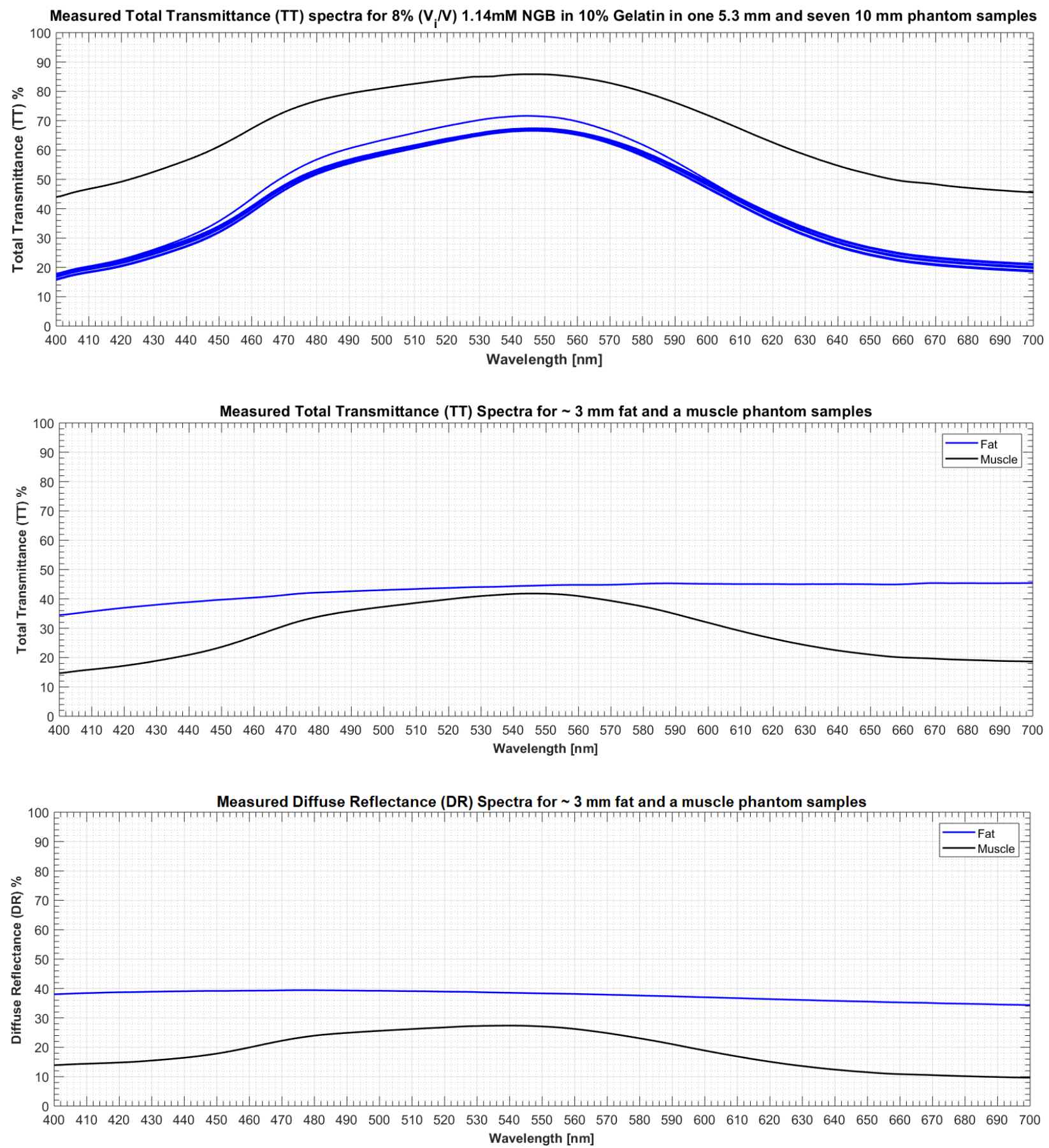

Figure D.1: TOP: Measured Total Transmittance spectra (TT)of $8 \% \frac{V_{i}}{V}$ Napthol Green (dye used for muscle absorption) in 10\% Gelatin for one $5.3 \mathrm{~mm}$ (BLACK) and seven 10 $\mathrm{mm}$ (BLUE) phantom samples. These measured spectra, via Beer-Lambart Law, were used to calculated the absorption spectra of the muscle phantom. MIDDLE: Measured TT spectra of the $\approx 3 \mathrm{~mm}$-thick fat and muscle phantom samples. BOTTOM: Measured Diffuse Reflectance $(\mathrm{DR})$ spectra of the $\approx 3 \mathrm{~mm}$-thick fat and muscle phantom samples. 


\section{Bibliography}

[1] Mensah, G., Norrving, B. and Feigin, V. (2015). The Global Burden of Stroke. Neuroepidemiology, 45(3), pp.143-145.

[2] Benjamin EJ, Blaha MJ, Chiuve SE, et al. on behalf of the American Heart Association Statistics Committee and Stroke Statistics Subcommittee, "Heart Disease and Stroke Statistics-2017 Update: A Report From the American Heart Association", Circulation. 2017;135:e229-e445.

[3] Mittmann, Nicole et al. "Impact Of Disability Status On Ischemic Stroke Costs In Canada In The First Year". The Canadian Journal Of Neurological Sciences, vol 39, no. 06,2012 , pp. 793-800.

[4] Rajsic, S., Gothe, H., Borba, H., Vujicic, J., Toell, T. and Siebert, U. (2016). Economic Burden of Stroke: A Systematic Review on Post-Stroke Care. Value in Health, 19(7), p.A633.

[5] Who.int. (2018). Ageing and health. [online] Available at: https://www.who.int/newsroom/fact-sheets/detail/ageing-and-health. [Accessed 01 Nov. 2018].

[6] Mozzafarian D, Benjamin EJ, Go AS, Arnett DK, Blaha MJ, Cushman M, et al., on behalf of the American Heart Association Statistics Committee and Stroke Statistics Subcommittee. Heart disease and stroke statistics2016 update: a report from the American Heart Association. Circulation 2016;133(4):e38360. 
[7] Yamada, Kiyofumi et al. "Asymptomatic Moderate Carotid Artery Stenosis With Intraplaque Hemorrhage: Onset Of New Ischemic Stroke". Journal Of Neurointerventional Surgery, vol 8, no. 2, 2014, pp. 130-134.

[8] Ninds.nih.gov. (2018). Stroke: Hope Through Research , National Institute of Neurological Disorders and Stroke. Available at: https://www.ninds.nih.gov/Disorders/PatientCaregiver-Education/Hope-Through-Research/Stroke-Hope-Through-Research [Accessed 29 Oct. 2018].

[9] Hobson, R., Mackey, W., Ascher, E., Murad, M., Calligaro, K., Comerota, A., Montori, V., Eskandari, M., Massop, D., Bush, R., Lal, B. and Perler, B. (2008). Management of atherosclerotic carotid artery disease: Clinical practice guidelines of the Society for Vascular Surgery. Journal of Vascular Surgery, 48(2), pp.480-486.

[10] Romero, Jos Rafael et al. "Stroke prevention: modifying risk factors Therapeutic advances in cardiovascular disease vol. 2,4 (2008): 287-303.

[11] Strokeassociation.org. (2018). Stroke Treatments. Available at: http://www.strokeassociation.org/STROKEORG/AboutStroke/BLS/StrokeTreatments_UCM_310892_Article.jsp [Accessed 01 Dec. 2018].

[12] Masoomi, Reza et al. "Progression Of External And Internal Carotid Artery Stenosis Is Associated With A Higher Risk Of Ischemic Neurologic Events In Patients With Asymptomatic Carotid Artery Stenosis". Vascular Medicine, vol 22, no. 5, 2017, pp. 418-423. SAGE Publications.

[13] Mughal, Majid M et al. "Symptomatic and Asymptomatic Carotid Artery Plaque." Expert review of cardiovascular therapy 9.10 (2011): 13151330. PMC.

[14] Mineva PP, Manchev IC, Hadjiev DI. Prevalence and outcome of asymptomatic carotid stenosis: a population-based ultrasonographic study. Eur J Neurol. 2002;9(4):383388. 
[15] Libby, Peter. "Atherosclerosis: The New View". Scientific American, vol 286, no. 5, 2002, pp. 46-55. Springer Nature, doi:10.1038/scientificamerican0502-46.

[16] W. van Lammeren, Guus et al. "Atherosclerotic Plaque Biomarkers: Beyond The Horizon Of The Vulnerable Plaque". Current Cardiology Reviews, vol 7, no. 1, 2011, pp. 22-27. Bentham Science Publishers Ltd., doi:10.2174/157340311795677680.

[17] Skagen, Karolina et al. "Unstable Carotid Artery Plaque: New Insights And Controversies In Diagnostics And Treatment". Croatian Medical Journal, vol 57, no. 4, 2016, pp. 311-320.

[18] Treiman, Gerald S. et al. "Correlation Of Carotid Intraplaque Hemorrhage And Stroke Using 1.5 T And 3 T Mri”. Magnetic Resonance Insights, vol 81, 2015, p. MRI.S23560.

[19] Wu, Baijian et al. "How Does Calcification Influence Plaque Vulnerability? Insights From Fatigue Analysis". The Scientific World Journal, vol 2014, 2014, pp. 1-8. Hindawi Limited, doi:10.1155/2014/417324.

[20] Brinjikji, Waleed et al. "The Association Between Carotid Intraplaque Hemorrhage And Outcomes Of Carotid Stenting: A Systematic Review And Meta-Analysis". Journal Of Neurointerventional Surgery, vol 9, no. 9, 2016, pp. 837-842.

[21] Altaf, Nishath et al. "Carotid Intraplaque Hemorrhage Predicts Recurrent Symptoms In Patients With High-Grade Carotid Stenosis". Stroke, vol 38, no. 5, 2007, pp. 1633-1635.

[22] Scott McNally, J., Yoon, H., Kim, S., Narra, K., McLaughlin, M., Parker, D. and Treiman, G. (2014). Carotid MRI Detection of Intraplaque Hemorrhage at 3T and 1.5T. Journal of Neuroimaging, 25(3), pp.390-396.

[23] Hosseini, Akram A. et al. "Carotid Plaque Hemorrhage On Magnetic Resonance Imaging Strongly Predicts Recurrent Ischemia And Stroke". Annals Of Neurology, vol 73, no. 6, 2013, pp. 774-784. 
[24] Ostwald, S., Godwin, K., Ye, F. and Cron, S. (2013). Serious Adverse Events Experienced by Survivors of Stroke in the First Year Following Discharge from Inpatient Rehabilitation. Rehabilitation Nursing, 38(5), pp.254-263.

[25] Lukanova, D., Nikolov, N., Genova, K., Stankev, M. and Georgieva, E. (2015). "The Accuracy of Noninvasive Imaging Techniques in Diagnosis of Carotid Plaque Morphology." Open Access Macedonian Journal of Medical Sciences, 3(2), p.224.

[26] Libby, Peter. "Inflammation In Atherosclerosis". Nature, vol 420, no. 6917, 2002, pp. 868-874. Springer Nature, doi:10.1038/nature01323.

[27] Leung, General, and Alan R. Moody. "MR Imaging Depicts Oxidative Stress Induced By Methemoglobin”. Radiology, vol 257, no. 2, 2010, pp. 470-476.

[28] L. Randeberg, J. Bonesronning, M. Dalaker, J. Nelson, L. Svaasand, "Methemoglobin formation during laser induced photothermolysis of vascular skin lesions". Lasers in Surgery and Medicine 34 (2004), 414-419.

[29] Omlc.org. (2019). Optical Absorption of Hemoglobin. [online] Available at: https://omlc.org/spectra/hemoglobin/ Accessed [01 May. 2016].

[30] A. B. Bell, "On the production and reproduction of sound by light," Am. J. Sci. 20, 305 (1880).

[31] Xia, Jun et al. "Photoacoustic tomography: principles and advances," Electromagnetic waves (Cambridge, Mass.) vol. 147 (2014): 1-22.

[32] Zhou, Y., Yao, J. and Wang, L. (2016). "Tutorial on photoacoustic tomography." Journal of Biomedical Optics, 21(6), p.061007.

[33] Beard, P. (2011). " Biomedical photoacoustic imaging.” Interface Focus, 1(4), pp.602631. 
[34] Recendt.at. (2016). Photoacoustics - RECENDT Research Center for Non Destructive Testing GmbH. [online] Available at: https://www.recendt.at/en/PA.html [Accessed 15 May. 2016].

[35] Keiser G. (2016) Light-Tissue Interactions. In: Biophotonics. Graduate Texts in Physics. Springer, Singapore.

[36] Jacques, S. (2013). Optical properties of biological tissues: a review. Physics in Medicine and Biology, 58(11), pp.R37-R61.

[37] Wang, Lihong V and Song Hu. Photoacoustic tomography: in vivo imaging from organelles to organs Science (New York, N.Y.) vol. 335,6075 (2012): 1458-62.

[38] Mallidi, Srivalleesha et al. Photoacoustic imaging in cancer detection, diagnosis, and treatment guidance Trends in biotechnology vol. 29,5 (2011): 213-21.

[39] Mehrmohammadi, Mohammad et al. Photoacoustic Imaging for Cancer Detection and Staging Current molecular imaging vol. 2,1 (2013): 89-105.

[40] Valluru, Keerthi S and Juergen K Willmann. Clinical photoacoustic imaging of cancer Ultrasonography (Seoul, Korea) vol. 35,4 (2016): 267-80.

[41] Hysi, E., Wirtzfeld, L., May, J., Undzys, E., Li, S. and Kolios, M. (2017). "Photoacoustic signal characterization of cancer treatment response: Correlation with changes in tumor oxygenation." Photoacoustics, 5, pp.25-35.

[42] May, J., Hysi, E., Wirtzfeld, L., Undzys, E., Li, S. and Kolios, M. (2016). "Photoacoustic Imaging of Cancer Treatment Response: Early Detection of Therapeutic Effect from Thermosensitive Liposomes." PLOS ONE, 11(10), p.e0165345.

[43] Hysi, E., Saha, R. and Kolios, M. (2012). "Photoacoustic ultrasound spectroscopy for assessing red blood cell aggregation and oxygenation." Journal of Biomedical Optics, 17(12), p.125006. 
[44] Xia, Wenfeng et al. "Interventional Photoacoustic Imaging of the Human Placenta with Ultrasonic Tracking for Minimally Invasive Fetal Surgeries" Medical image computing and computer-assisted intervention : MICCAI ... International Conference on Medical Image Computing and Computer-Assisted Intervention vol. 9349 (2015): 371-378.

[45] Jo, J., Xu, G., Cao, M., Marquardt, A., Francis, S., Gandikota, G. and Wang, X. (2017). "A Functional Study of Human Inflammatory Arthritis Using Photoacoustic Imaging." Scientific Reports, 7(1).

[46] Dima, A. and Ntziachristos, V. (2012). "Non-invasive carotid imaging using optoacoustic tomography." Optics Express, 20(22), p.25044.

[47] Jansen, Krista et al. "Intravascular Photoacoustic Imaging: A New Tool For Vulnerable Plaque Identificaton". Ultrasound In Medicine Biology, vol 40, no. 6, 2014, pp. 10371048. Elsevier BV.

[48] Wang, B., Karpiouk, A., Yeager, D., Amirian, J., Litovsky, S., Smalling, R., and Emelianov, S. "In vivo intravascular ultrasound-guided photoacoustic imaging of lipid in plaques using an animal model of atherosclerosis." Ultrasound Med Biol. 2012; 38: 20982103

[49] Arabul, M. Umit et al. "Ex vivo photoacoustic imaging of atherosclerotic carotid plaques. 2015 IEEE International Ultrasonics Symposium (IUS) (2015): 1-4.

[50] Zafar, Haroon et al. "Photoacoustic Cardiovascular Imaging: A New Technique For Imaging Of Atherosclerosis And Vulnerable Plaque Detection”. Biomedical Physics Engineering Express, vol 4, no. 3, 2018, p. 032002. IOP Publishing, doi:10.1088/20571976/aab640.

[51] Zhu, Dan et al. Recent progress in tissue optical clearing Laser photonics reviews vol. 7,5 (2013): 732-757. 
[52] Luke, G., Yeager, D. and Emelianov, S. (2011). "Biomedical Applications of Photoacoustic Imaging with Exogenous Contrast Agents." Annals of Biomedical Engineering, 40(2), pp.422-437.

[53] Omlc.org (2016) Skin Optics Summary. [online] Available at: https://omlc.org/news/jan98/skinoptics.html [Accessed 16 July. 2016].

[54] Training.seer.cancer.gov. (2018). "Layers of the Skin — SEER Training". [online] Available at: https://training.seer.cancer.gov/melanoma/anatomy/layers.html [Accessed 20 Feb. 2018].

[55] Bashkatove, A., Genina, E. and Tuchin, V. (2011). "Optical Properties of skin, subcutaneous and muscle tissues: a Review." Journal of Innovative Optical Health Sciences, 04(01), pp.9-38.

[56] van Veen, R., Sterenborg, H., Pifferi, A., Torricelli, A., Chikoidze, E. and Cubeddu, R. (2005). "Determination of visible near-IR absorption coefficients of mammalian fat using time- and spatially resolved diffuse reflectance and transmission spectroscopy." Journal of Biomedical Optics, 10(5), p.054004.

[57] Jacques, S. (2014). "Coupling 3D Monte Carlo light transport in optically heterogeneous tissues to photoacoustic signal generation." Photoacoustics, 2(4), pp.137-142.

[58] Amato, Mauro et al. "Carotid Plaque-Thickness And Common Carotid IMT Show Additive Value In Cardiovascular Risk Prediction And Reclassification". Atherosclerosis, vol 263, 2017, pp. 412-419. Elsevier BV, doi:10.1016/j.atherosclerosis.2017.05.023.

[59] Nachab, Rami et al. "Estimation Of Biological Chromophores Using Diffuse Optical Spectroscopy: Benefit Of Extending The UV-VIS Wavelength Range To Include 1000 To 1600 Nm". Biomedical Optics Express, vol 1, no. 5, 2010, p. 1432. The Optical Society, doi:10.1364/boe.1.001432. 
[60] Konugolu Venkata Sekar, Sanathana et al. "Broadband Diffuse Optical Characterization Of Elastin For Biomedical Applications". Biophysical Chemistry, vol 229, 2017, pp. 130134. Elsevier BV, doi:10.1016/j.bpc.2017.07.004.

[61] Johnson, Jami L et al. "All-optical extravascular laser-ultrasound and photoacoustic imaging of calcified atherosclerotic plaque in excised carotid artery Photoacoustics vol. 9 62-72. 7 Feb. 2018, doi:10.1016/j.pacs.2018.01.002

[62] Bona, Adriana et al. "Laser Technology For Caries Removal". Contemporary Approach To Dental Caries, 2012. Intech, doi:10.5772/37227. Accessed 25 Jan 2019.

[63] Prahl, S. (2016) Omlc.org. . mcxyz.c. Available at: https://omlc.org/software/mc/mcxyz/index.html [Accessed 15 Jan. 2016].[ Accessed 15 July 2018].

[64] Vogt, William C et al. "Biologically relevant photoacoustic imaging phantoms with tunable optical and acoustic properties" Journal of biomedical optics vol. 21,10 (2016): 101405.

[65] "Optical Absorption Of Intralipid." Omlc.Org, 2019, https://omlc.org/spectra/intralipid/. [Accessed 8 June 2017.]

[66] Sigma-Aldrich: Analytical, Biology, Chemistry Materials Science Products And Services. Sigma-Aldrich, 2019, https://www.sigmaaldrich.com/. [Accessed 8 Jan 2019.]

[67] S.A. Prahl, M.J.C. van Gemert, and A.J. Welch, "Determining the optical properties of turbid media by using the adding-doubling method," Applied Optics 32, 559568 (1993).

[68] S.A. Prahl, Inverse Adding-Doubling program: Version 3-9-6, (2013). (http://omlc.ogi.edu/software/iad/index.html) [ Accessed Jan. 4 2019].

[69] S. Prahl, "Everything I think you should know about inverse adding-doubling," Oregon Medical Laser Center, St. Vincent Hospital 174 (2011). 
[70] S.A. Prahl, "Light Transport in Tissue", (The University of Texas at Austin, 1988).

[71] Ingle, J. and Crouch, S. (1988). "Spectrochemical analysis." New Jersey: Prentice Hall.

[72] Prahl, S. (2017). Methylene Blue Spectra. [online] Omlc.org. Available at: https://omlc.org/spectra/mb/ [Accessed 2 Mar. 2016].

[73] Flock, Stephen T. et al. "Optical Properties Of Intralipid: A Phantom Medium For Light Propagation Studies." Lasers In Surgery And Medicine, vol 12, no. 5, 1992, pp. $510-519$.

[74] Keszler, Agnes et al. The reaction between nitrite and oxyhemoglobin: a mechanistic study Journal of biological chemistry vol. 283,15 (2008): 9615-22.

[75] Cook, Jason R et al. "Tissue-mimicking phantoms for photoacoustic and ultrasonic imaging Biomedical optics express vol. 2,11 (2011): 3193-206.

[76] Skilton, Michael R. et al. "Carotid IntimaMedia And Adventitial Thickening: Comparison Of New And Established Ultrasound And Magnetic Resonance Imaging Techniques". Atherosclerosis, vol 215, no. 2, (2011), pp. 405-410.

[77] G Yoon. "Absorption and scattering of laser light in biological media - mathematical modeling and methods for determining optical properties." Ph.D. thesis, Univ. of Texas at Austin, 1988.

[78] Krejza, Jaroslaw et al. "Carotid Artery Diameter In Men And Women And The Relation To Body And Neck Size". Stroke, vol 37, no. 4, 2006, pp. 1103-1105.

[79] Cannon, C. G., and I. S. C. Butterworth. "Beer's Law And Spectrophotometer Linearity". Analytical Chemistry, vol 25, no. 1, 1953, pp. 168-170. American Chemical Society (ACS).

[80] Wang, Jing et al. "Saturation effect in functional photoacoustic imaging Journal of biomedical optics vol. 15,2 (2010): 021317. 
[81] J. L. Prince and J. M. Links, Medical Imaging Signals and Systems (Pearson Prentice Hall, 2006).

[82] Allman, Derek et al. "Photoacoustic Source Detection And Reflection Artifact Removal Enabled By Deep Learning". IEEE Transactions On Medical Imaging, vol 37, no. 6, 2018, pp. 1464-1477. Institute Of Electrical And Electronics Engineers (IEEE), doi:10.1109/tmi.2018.2829662.

[83] Michailovich, Oleg V and Allen Tannenbaum. "Despeckling of medical ultrasound images IEEE transactions on ultrasonics, ferroelectrics, and frequency control vol. 53,1 (2006): 64-78.

[84] Guo, Zijian et al. "On the speckle-free nature of photoacoustic tomography Medical physics vol. 36,9 (2009): 4084-8.

[85] American National Standards Institute, American National Standard for the Safe Use of Lasers in Health Care Facilities: Standard Z136.1-2014 (ANSI, Inc., New York, 2000).

[86] "CUDA - Geforce". Geforce.Com, 2018, https://www.geforce.com/hardware/technology/cuda. [Accessed 11 May 2018.]

[87] Ash, Caerwyn et al. "Effect Of Wavelength And Beam Width On Penetration In LightTissue Interaction Using Computational Methods". Lasers In Medical Science, vol 32, no. 8, 2017, pp. 1909-1918. Springer Nature, doi:10.1007/s10103-017-2317-4. 